



\section{Vibration Measurement of a Model Wind Turbine Using High Speed Photogrammetry}

A thesis submitted to the Delft University of Technology in partial fulfilment of the requirements for the degree of

Master of Science in Geomatics

by

Dinesh Sharma Kalpoe

November 2010

Supervisors: Prof. dr. Massimo Menenti

Dr. Kourosh Khosh Elham

Dr.ir. Ben G.H. Gorte

Co-reader : Dr. Hugo Ledoux 
Dinesh S. Kalpoe: Vibration measurement of a model wind turbine using high speed photogrammetry, MSc thesis, Delft University of Technology, November 2010

\section{LOCATION:}

\section{T̛́UDelft}

Delft University of Technology

Department of Optical and Laser Remote Sensing

Faculty of Aerospace Engineering

Delft University of Technology

The Netherlands

TIMEFRAME:

December 2009 - November 2010

FINAL PRESENTATION:

16 November 2010

EXAMINATION COMMITTEE:

Prof. dr. ing. R. Klees

Prof. dr. M. Menenti

Dr. K. Khosh Elham

Dr.ir. B.G.H. Gorte

Dr. H. Ledoux

\section{COVER ILLUSTRATION:}

The cover shows the vibrations of a wind turbine measured from images. The blue dots are target positions measured in a sequence of images. The two dotted red lines are the tracks of two targets. The magenta line shows an exaggerated vibration of the most outer target. 


\section{Abstract}

Energy produced from wind is one of the techniques that is still being used to successfully produce green energy. To meet the growing demand for green energy, existing wind turbines have to be made more powerful and efficient. To make wind turbines more efficient and durable, research is done on the aero-elastic stability limits of the blades. Aero-elastics of wind turbines can be analyzed by the vibrations that occur on the blade during normal operational conditions. Until now several methods have been used to measure the vibrations of a wind turbine such as strain accelerometers, strain gauges and Laser Doppler Vibrometers. Because all these techniques are limited to some extent, a new technique has been investigated in this study to measure the vibrations using high speed photogrammetry .

By placing targets on the blades of the wind turbine and taking images with two synchronized high speed cameras, the motion of the blades is captured. High speed cameras produce a large number of images, making manual target measurements impractical. In this study, it is investigated how target measurements in high speed images can be automated and what accuracy can be obtained for the reconstructed the object coordinates.

To automate the target measurements, a method is developed based on target detection and tracking. Since the targets describe a circular motion a circle can be fitted to the first three positions of a target and after that its position can be predicted in the next frames. The targets in the first three frames are detected using a threshold histogram segmentation. To establish correspondence between the targets, four constraints of a circular motion are used. To obtain a target measurement with subpixel precision, the edges from the target are detected and a circle is fitted to the edges.

To reconstruct the object coordinates a Direct Linear Transformation (DLT) is used. The equitations of DLT are linear and can easily be solved with standard Least Squares Estimation (LSQ). To solve the DLT parameters a minimum of six reference points is needed, which is not a limitation for this application.

An experiment was performed on a model wind turbine placed in a wind 
tunnel with thirty two retro-reflective targets placed on the blades. The images were acquired with a high speed camera of $500 \mathrm{~Hz}$ at a rotational speed of 260 rotations per minute (rpm).

Using the images of the experiment a robustness analysis was carried out for the developed method for automatic target measurement and tracking. From this analysis, it could be concluded that targets can be precisely measured and tracked in high speed images works, even in the presence of outlying features or noise. The target tracking and establishing correspondence could be performed successfully for an acquisition frequency of $125 \mathrm{~Hz}$ and higher.

Since no reference measurements were made available for this study, the proposed DLT method could not be directly used. Instead, two other software packages were used, namely PhotoCore to measure reference measurements and PhotoModeler to perform a relative orientation to calculate model coordinates in an arbitrary scale. These model coordinates were used to calculate DLT parameters, from which model coordinates were calculated of the targets in all images. Using the reference targets, an affine transformation was obtained and used to apply an absolute orientation. The final 3D object coordinates had a accuracy of 1.32 millimeter.

The vibrations of a target were obtained by means of Principal Component Analysis (PCA). Using PCA, the track of coordinates of a target were transformed to a new coordinate system. The $x y$ plane of this new coordinate system coincides with the rotation plane of the target and the $z$ is perpendicular to it, containing the vibrations. The measured vibration had a maximum amplitude of $6 \mathrm{~mm}$, with an accuracy of $0.56 \mathrm{~mm}$ in the vibration component.

From the performed experiment with the model wind turbine, it can be concluded that the blade vibrations can be measured using high speed photogrammetry. 


\section{Acknowledgments}

I am grateful to everyone who helped me during my study for this thesis, which finally resulted in this report. First of all I would like to thanks my supervisors Kourosh Khosh Elham and Ben Gorte for their guidance, valuable suggestions and continuous support. Kourosh was always kind enough to make time for me to debug my MATLAB code. I am grateful for all the help of Ben with the final calculations of this study. I also would like to thank my graduation professor Massimo Menenti for his valuable remarks to improve the results of this study.

Additionally, I would like to thank Roderik Lindenbergh for his advice on mathematical problems which I encountered during my thesis research. Lidwien de Jong who gave me access to the calenders of my supervisors. Hugo Ledoux who was kind enough to become the co-reader of my report.

I also would like to thank my fellow Geomatics students whose companionship made my study time in Delft a pleasant experience. My office mates Hang Yu and Stijn Verlaar, with whom I had interesting discussions on several aspects of photogrammetry. My special thanks to Ken Arroyo Ohori who helped me to learn programming during the first year of my study, the valuable discussion we had regarding this thesis and his help with the layout of my report.

As last I would like to thank my family members for their support. My partner Rashmi Sahtoe for all her patience to read my report several times to correct for grammatical errors. And especially for her continuous support during these two years.

Thank you all. 


\section{Contents}

1 Introduction $\quad 1$

1.1 Motivation . . . . . . . . . . . . . . . . 1

1.2 Problem Statement . . . . . . . . . . . . . . . . . . 3

1.3 Research Objective and Questions . . . . . . . . . . . . . . . 3

1.4 Related Research . . . . . . . . . . . . . . . . . . . . 4

1.5 Thesis Outline . . . . . . . . . . . . . . . 5

2 Vibration Measurement of a Wind Turbine $\quad 7$

2.1 Vibrations of Wind Turbines . . . . . . . . . . . . 7

2.2 Review of Vibration Measurement Techniques . . . . . . . . . 8

2.3 Comparison of Vibration Measurement Techniques . . . . . . . 10

3 High Speed Photogrammetric Measurement 13

3.1 High Speed Imaging . . . . . . . . . . . . . . . . . . . . 14

3.1 .1 High Speed Cameras . . . . . . . . . . . . . . . 14

3.1.2 Retro-Reflective Targets . . . . . . . . . . . 16

3.2 Automatic Target Measurement in Digital Images . . . . . . . 18

3.2.1 Target Measurement in Individual Images . . . . . . . 18

3.2.2 Establishing Correspondence Between Multiple Images 23

3.3 Mathematical Fundamentals of Photogrammetry . . . . . . . . 28

3.3.1 Coordinate Systems . . . . . . . . . . . . . . . . 29

3.3 .2 Interior Orientation . . . . . . . . . . . . . . 31

3.3.3 Exterior Orientation . . . . . . . . . . . . 33

3.3.4 Collinearity Equations . . . . . . . . . . . . . . . . 38

3.3.5 Direct Linear Transformation . . . . . . . . . . . . . 39

3.3.6 3D Object Reconstruction . . . . . . . . . . . . 40

3.4 Vibration Measurement of Reconstructed 3D Position . . . . . 41

4 Automated Target Measurement and Tracking 45

4.1 Workflow of Automatic Target Tracking . . . . . . . . . . . . 45

4.2 Target Detection . . . . . . . . . . . . . . 47 
4.3 Removing Reference Targets . . . . . . . . . . . . . . . . . . 48

4.4 Establishing Correspondence . . . . . . . . . . . . . . . 50

4.5 Model Based Target Tracking . . . . . . . . . . . . . . . 53

4.6 Target Measurement With SubPixel Precision . . . . . . . . 53

4.6.1 Edge Detection . . . . . . . . . . . . . 55

4.6.2 Pin-Pointing Target Center . . . . . . . . . . 55

4.6.3 Eliminating Outlying Edge Pixels using RANSAC . . . 56

4.6.4 Circle and Ellipse Fitting . . . . . . . . . . . . . 58

4.7 Accuracy Assessment of Method . . . . . . . . . . . . . . . 62

5 Experimental Results $\quad 65$

5.1 Experimental Setup . . . . . . . . . . . . . . . 65

5.2 Results . . . . . . . . . . . . . . . . . . 68

5.2.1 Results of Automatic Target Measurement and Tracking 70

5.2.2 Calculated Object Coordinates . . . . . . . . . . 72

5.2.3 Results of Vibration Measurement . . . . . . . . . . 76

5.2 .4 Discussion on Measured Vibration . . . . . . . . . 77

6 Performance Analysis of The Methods $\quad 81$

6.1 Robustness of Automated Target measurement . . . . . . . . 81

6.1.1 Target detection . . . . . . . . . . . . 81

6.1.2 Removing Reference Targets . . . . . . . . . . . . . 83

6.1.3 Establishing Correspondence . . . . . . . . . . . . 83

6.1.4 Model Based Target Tracking . . . . . . . . . . . . 85

6.1.5 Precise Target Measurement . . . . . . . . . . . 86

6.1.6 Comparison of Circle Versus Ellipse Fitting . . . . . . 88

6.1.7 Performance Target Tracking . . . . . . . . . . . . . 89

6.2 Accuracy Assessment of the Results . . . . . . . . . . . . . . . 89

6.2.1 Accuracy of Target Measurement . . . . . . . . . . . . 91

6.2.2 Accuracy of Relative Orientation . . . . . . . . . . . . . 93

6.2.3 Accuracy of Measured Reference Points . . . . . . . . . 95

6.2.4 Accuracy of 3D Reconstructed Target Coordinates . . . 97

6.3 Accuracy of derived Vibrations . . . . . . . . . . . . . . 98

7 Conclusions and Recommendations 101

7.1 Conclusions . . . . . . . . . . . . . . . . . . . . 101

7.2 Recommendations . . . . . . . . . . . . . . . . . . 102

$\begin{array}{ll}\text { Bibliography } & 105\end{array}$ 


\section{List of Figures}

1.1 Wind turbine park at the Afsluitdijk in Noord Holland . . . . 2

3.1 The photogrammetric process from object to model . . . . . . 14

3.2 Bucket array to capture electromagnetic energy . . . . . . . . 15

3.3 Main elements of a high speed camera . . . . . . . . . . . 16

3.4 Image made with a high speed camera of circular retro-reflective targets . . . . . . . . . . . . . . . 17

3.5 The principle of retro-reflective targets . . . . . . . . . . 17

3.6 Image segmented by histogram threshold . . . . . . . . . . . . 21

3.7 Edge detection using Canny edge detection method . . . . . . 22

3.8 An example of two coded targets . . . . . . . . . . . . . 24

3.9 Shear between matching targets . . . . . . . . . . . . . 25

3.10 Least squares matching between two overlapping images . . . 26

3.11 Example of least squares image matching applied on high speed images . . . . . . . . . . . . . . . . . 27

3.12 Tracking of human motion . . . . . . . . . . . . . . . . 28

3.13 The coordinate systems used in photogrammetry . . . . . . 30

3.14 Interior geometry of a camera . . . . . . . . . . . . . . 32

3.15 External orientation of a camera . . . . . . . . . . . . 33

3.16 Relative orientation of the two stereo images . . . . . . . . . 35

3.17 Absolute orientation of a model coordinate system to the object coordinate system . . . . . . . . . . . . 37

3.18 The principal components of the tracked positions of a target to derive flapwise vibrations . . . . . . . . . . . 43

4.1 Flow diagram for automated target measurement and tracking 46

4.2 Target detection based on histogram thresholding . . . . . . . 47

4.3 The calculated target centers of a binary segmented image . . 48

4.4 Reference and blade targets distinguished by a threshold of movement . . . . . . . . . . . . . . 49

4.5 Sensitivity of using nearest neighbor to establish correspondence 50 
4.6 Constraints to find corresponding targets in three successive images . . . . . . . . . . . . . . . . 52

4.7 Predicted target position using a LS fitted circle . . . . . . . 53

4.8 Unsuccessful tracking caused by imprecise targets measurement 54

4.9 Window size used to detect edges with Canny edge detector . 55

4.10 LSQ circle fitted to detected edges of a target . . . . . . . 56

4.11 Undesired features in a high speed image . . . . . . . . . . 57

4.12 Fitting a circle in the presence of noise . . . . . . . . . . . 57

4.13 Deformed shapes of a circular target . . . . . . . . . . 59

5.1 The wind turbine blade . . . . . . . . . . . . . 66

5.2 Overall layout of the wind tunnel with the dimensions of the experiment chamber . . . . . . . . . . 66

5.3 A schematic overview of the experimental setup . . . . . . 67

5.4 Experimental setup . . . . . . . . . . . . . . . 68

5.5 Pair of images acquired with two high speed cameras. For better visualization the color scale of the images are inverted from dark to white to white to dark . . . . . . . . . . 69

5.6 Acquisition setup of blade with reference targets to calculate its object coordinates using the software PhotoCore . . . . . . 69

5.7 Tracked and measured position of two targets in the sequence of 115 images are plotted in the first image of the sequence . . 71

5.8 The $x$ and $y$ coordinates of the two tracked targets . . . . 71

5.9 Object coordinates calculated with PhotoModeler by applying relative and absolute orientation . . . . . . . . . . 73

5.10 Model coordinates calculated of the sequence of images using the DLT parameters . . . . . . . . . . . . . 74

5.11 Automatically calculated object coordinates of coded and circular targets using the software PhotoCore . . . . . . . . 75

5.12 Absolutely oriented object coordinates of the targets . . . . . 75

5.13 The third principal component or derived vibration from the calculated object coordinated of two tracked targets . . . . . 76

5.14 Signal vibration transferred from time domain to frequency domain using Fast Fourier transformation . . . . . . . . 76

5.15 Vibration signal filtered with threshold frequency value . . . . 77

5.16 Circle fitted to the tracked coordinates of two targets in the rotational plane of the first and second principal component . 78

5.17 Distance between the calculated object coordinates of the two tracked targets and the fitted circle . . . . . . . . . 78

6.1 Robustness analysis of threshold value for image segmentation 82 
6.2 The motion of a target calculated for a range of rotation speeds and image acquisition frequencies . . . . . . . . . . 83

6.3 Robustness analysis of creating correspondence to determine acquisition frequency with acceptable error rate . . . . . . . 85

6.4 The mean and maximum deviations of the predicted location per target . . . . . . . . . . . . . . 86

6.5 Robustness analysis of target prediction to determine acquisition frequency with acceptable predictions . . . . . . . . 87

6.6 Robustness analysis to determine the range of acceptable template window sizes . . . . . . . . . . . . 87

6.7 The two closest targets identified to analyze the template window size . . . . . . . . . . . . . . . . . 88

6.8 Fitting an ellipse and a circle to a set of points to analyze the difference between the measured target centers . . . . . . . . . 90

6.9 Analyzing the correctness of the tracked target by fitting an ellipse . . . . . . . . . . . . . . . . . . 91

6.10 Artificial target created to estimate the accuracy by which the center of a target can be pin-pointed . . . . . . . . . . . 92

6.11 Residuals in image space after relative orientation . . . . . . . 93

6.12 Residuals in image space for the calculated DLT parameters . 94

6.13 Residuals in model space for the calculated DLT parameters . 96

6.14 Residual vectors of the reconstructed targets using PhotoCore 97

6.15 Residuals of absolute orientation . . . . . . . . . . . . . 98 


\section{List of Tables}

2.1 Comparison matrix of measuring techniques . . . . . . . . . 11

4.1 At a sample size of three the number of samples required at a certain proportion of outliers . . . . . . . . . 58

5.1 Calculated DLT parameters . . . . . . . . . . . 73

6.1 Performance of the correspondence constraints . . . . . . . . . 84

6.2 Results of difference between centers of a LSQ fitted circle and ellipse . . . . . . . . . . . . . . . . . . . . 88

6.3 Mean and standard deviation of the differences between the measured and actual position of an artificial target . . . . . 92

6.4 Residual vectors in image space obtained by relative orientation 93

6.5 Statistics of residuals in image space for the calculated DLT parameters . . . . . . . . . . . . . . . . . 95

6.6 Statistics of residuals in model space for the calculated of DLT parameters . . . . . . . . . . . . . . . . . . . . 95

6.7 Statistics of residuals in image space using PhotoCore . . . . . 96

6.8 Statistics of residuals in object space using affine transformation 97

6.9 Statistics of transformed residuals to the three principal components . . . . . . . . . . . . . . . . . . . . 99 


\section{Abbreviations}

3D Three Dimensional

CCD Charge-Coupled Device

DLT Direct Linear Transformation

fps Frames Per Second

LDV Laser Doppler Vibrometer

LSQ Least Squares Estimation

PCA Principal Component Analysis

RAM Random Access Memory

RANSAC Random Sample Consensus

rpm Rotations Per Minute

Std Standard Deviation 


\section{Chapter 1}

\section{Introduction}

\subsection{Motivation}

Wind turbines are one of the icons of the Dutch landscape as can be seen in the picture taken at the Afsluitdijk in North Holland (Figure 1.1). Wind turbines are able to convert kinetic energy of the air (wind) into rotational energy of the blades, which was used in the past for various purposes, such as pumping water, grinding wheat, sawing wood and is now mainly used for generating electrical energy (Brush, 2008). Energy production with wind turbines produces no waste, making this an environment friendly energy source. With the increasing demand for sustainable energy more pressure is put to improve and further develop wind turbines.

A research topic to make wind turbines more powerful and efficient in producing electrical energy is related to the aero-elastic stability limits of flexible wind turbines. Research about aero-elastic stability limits is interesting because it provides insights about structural stability and the life span of wind turbines. Aero-elastic models are used for two purposes (Rasmussen, 2003):

- to analyze the load stresses that a wind turbine can take, such as fatigue and load spectra to estimate the fatigue lifetime;

- to design and optimize new turbines, a detailed understanding of aeroelastic response characteristics is required, e.g. the stability of a wind turbine is vital for its behavior and operational lifetime.

The aero-elasticity of wind turbines can be physically translated as the maximum stress the blades of a turbine can take without breaking. The stress on the blades manifests itself in the form of vibrations. The measurements 


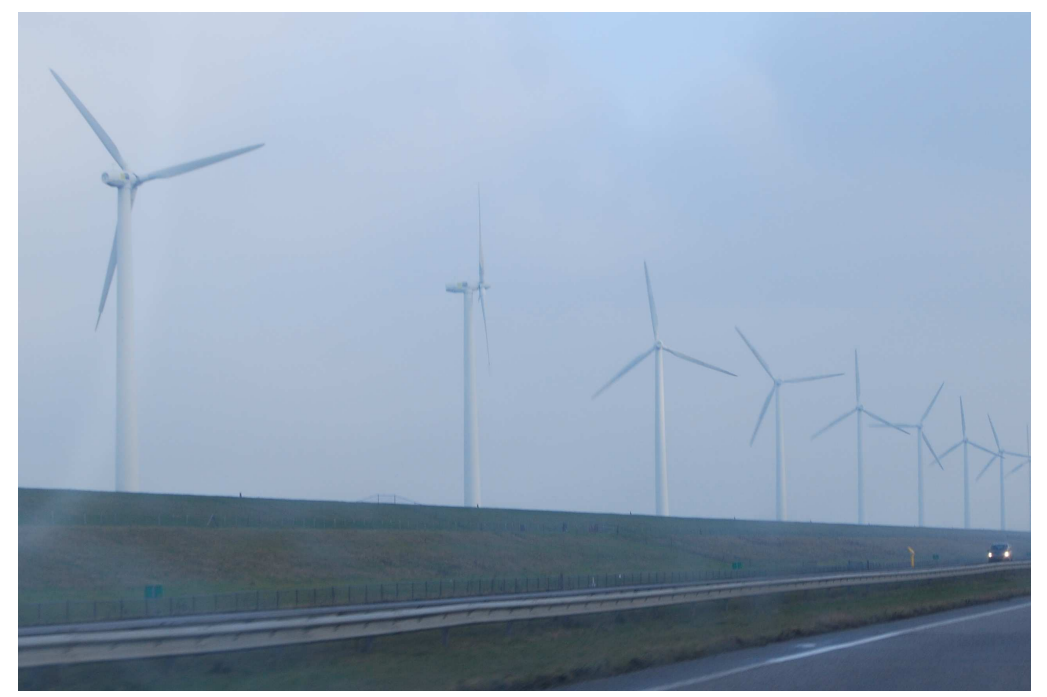

Figure 1.1: Wind turbine park at the Afsluitdijk in Noord Holland

of the vibrations of the blades at normal operational speed are necessary for studying the aero-elastic stability of the blade (Meng, 2009).

Basic studies of aero-elastic stability of wind turbines are usually performed on scale models put in a wind tunnel with controlled conditions. In such an experiment the vibrations can be measured at various wind speeds and rotation speeds. Measuring the vibration of a scale model wind turbine it should be kept in mind that the rotational speed and the vibration frequencies are larger compared to an full scaled wind turbine.

At present, accelerometers, strain gauges, fiber optic strain gauges and Laser Doppler Vibrometers (LDVs) are systems, that are commonly used to measure vibrations of a wind turbine. When accelerometers are put on the blades, they influence the aero-dynamics of the blades. The strain gauges are placed inside the blades, which makes installing of cables for energy and data transfer difficult around a rotation axis. LDVs are used remotely, but the blade has to be static. In order to have an optimal vibration measurement system, it has to be a non contact measurement system and it should be able to measure at normal operational speed. A measurement system that fulfills these criteria is high speed photogrammetry (Ozbek et al., 2010).

High speed photogrammetry is able to capture images of a wind turbine remotely without influencing the aero-dynamics of the blades. Images can be measured with an acquisition frequency up to 5000 Frames Per Second (fps) (GOM, 2010) enabling the system to measure the vibrations while in motion. 


\subsection{Problem Statement}

According to the sampling theory of Shannon (1949), a signal can be fully reconstructed if it is sampled with a frequency that is equal to or larger than twice the frequency bandwidth of the signal. If a wind turbine has a vibration frequency of $250 \mathrm{~Hz}$, it needs to be measured with an acquisition frequency of at least $500 \mathrm{fps}$.

On the other hand, to be able to understand dynamical phenomena it is also important to measure the motion for a relatively long time. Measuring with a high acquisition frequency for a relatively long time results in a large amount of data. For example, if the vibrations are measured with two high speed cameras with an acquisition frequency of $500 \mathrm{~Hz}$ for 1.5 seconds, they will produce 1500 images. And if in each image 50 targets are measured, than for all images 75000 targets need to be measured. Since manual measurement of this large amount of targets is impossible, it is necessary to automate the target measurement.

\subsection{Research Objective and Questions}

In order to get a better understanding of the aero-elastic stability limits of a flexible wind turbine, blade vibrations need to be measured at normal operating conditions.

The main objective of this research is to investigate and develop methods to automate the process of measuring vibrations of the blades of a wind turbine using high speed photogrammetry.

To achieve the main goal four research questions are formulated, namely:

1. how can the target measurement in the images be automated?

2. is it possible to track the targets in a sequence of images and how reliable is it?

3. what factor influences the performance of automated target measurement?

4. what is the accuracy of the target measurement in image space and object space for a typical setup? 


\subsection{Related Research}

In the study of Shortis et al. (1994), an analysis was conducted to measure circular targets in digital images with subpixel accuracy. The study demonstrated that precise target measurement can be done in two steps, namely target recognition and precise localization. The targets in an image can be detected using predefined patterns or by image segmentation. Once targets are identified their centers can be located precisely using edge fitting. Centroid and ellipse fitting were demonstrated to be the best methods to precisely determine the center of large binary targets. Furnee et al. (1997) also demonstrated that circle fitting can be used to precisely determine target centers.

Studying dynamical phenomena requires that correspondence has to be established between targets measured in a sequence of images. This correspondence problem was studied by Ariyawansa and Clarke (1997). For a fixed camera registering moving targets two solutions were described:

- using unique markers. By using coded targets or unique markers these can be recognized unambiguously. The disadvantage of this method is the large amount of unique targets that needs to be recognized in each image. The coded targets also limit the angle of view to prevent perspective distortions;

- target tracking. This relies on the fact that the time between successive images is short enough to capture the motion without any discontinuities. Target tracking can be carried out in different ways, e.g. with Kalman filtering, velocity or direction prediction.

A model based tracking was performed successfully by Kolahi et al. (2007) in a research of tracking marker based human motion. The model based tracking used was performed in two steps. First, correspondence was created between targets of the first few frames by fitting a representative motion model. Once the model was determined, it was used to predict the location of the targets in the next frames. In the research a biomechanical kinematic constraint was used, implying that accelerations of a moving human body may have no discontinuities. This was assured by a continuous second derivation of the motion.

Once the targets are measured in the images, the next step is to reconstruct its object coordinates. In traditional photogrammetry, collinearity equations are the basis for camera calibration and object reconstruction. In recent studies of motion monitoring with high speed videogrammetric systems, another method common in machine vision is used, namely Direct 
Linear Transformation (DLT). The equations of DLT are obtained by rearranging and combining terms of the collinearity equations. DLT consists of 11 parameters, is linear and no initial values are needed. Burner et al. (2001) studied DLT to determine the static and dynamic aero-elastic deformation of wind tunnel models. An optimized DLT was used, as developed by Tianshu et al. (2000), to increase the to accuracy of the camera calibration. More recent studies where DLT was used include the study of Hedrick et al. (2004) who studied the swimming motions of fish and Zheng et al. (2009) who studied the flight mechanisms of insects.

\subsection{Thesis Outline}

The structure of this report is as follows:

- In Chapter 2 background information is given about vibrations of a wind turbine. A review is made of the different methods to measure the vibrations and the most appropriate method is selected.

- The photogrammetric process is described in Chapter 3. This consists of the imaging system, methods for automated target measurement in digital images and the mathematics for object reconstruction.

- Chapter 4 discusses the developed method for automated target measurement and tracking in a sequence of images.

- In Chapter 5 the setup and results of the performed experiments are described.

- Using the images of the experiment a robustness analysis is performed of the automated target measurement and the accuracy is assessed of the calculated object coordinates in Chapter 6 .

- The conclusions and recommendations are provided in Chapter 7. 


\section{Chapter 2}

\section{Vibration Measurement of a Wind Turbine}

\subsection{Vibrations of Wind Turbines}

A wind turbine is a rotating machine which converts the kinetic energy of wind into mechanical energy. If the mechanical energy is directly used by machinery, such as a pump or grinding stone, the machine is called a windmill. If the mechanical energy is converted to electrical energy, the machine is called a wind generator or wind turbine (Brush, 2008).

Wind turbines are usually mounted on a tower of 30 meters or more above the ground, where they can capture energy from fast and less turbulent winds. The energy of the wind is caught with the propeller-like blades of the turbine. Usually, two or three blades are mounted on a shaft to form a rotor (energy world.com, 2009). The blades act much like an aeroplane airplane wing. When the wind blows, a pocket of low-pressure air forms on the downwind side of the blade. The low-pressure air pocket then pulls the blade towards it, causing the rotor to turn. This is called lift. The force of the lift is actually much stronger than the wind's force against the front side of the blade, which is called drag. The combination of lift and drag causes the rotor to spin like a propeller, and the turning shaft spins a generator to make electricity (energy world.com, 2009).

Traditionally, wind turbine rotors were designed without considering any aero-elastic stability properties. However, in the early 1990s some first stability problems appeared in the form of severe edgewise vibrations (Riziotis et al., 2004). These damages could not be explained because earlier research about aero-dynamic damping were mainly focused on the critical flapping mode. The reason why stability problems appeared at that time was be- 
cause of the new emerging blade designs and manufacturing technologies. Following the demand for lighter blades, new composite materials and advanced fabrication methods resulted in reduced structural damping. On the other side, abandoning geometric similarity in an attempt to scale up with increased performance and lower weight led to an uncontrollable decrease in aero-dynamic damping. The combination of these two led to disastrous instability in modern wind turbines. Because of the risk of vibrations, a new era started in wind turbine research to understand aero-elastic stability properties in order to make better wind turbines to meet the new demands (Riziotis et al., 2004).

\subsection{Review of Vibration Measurement Tech- niques}

According to the sampling theory of Shannon (1949), a signal can be completely reconstructed if its sampling frequency is equal or larger than twice the frequency of the signal. So in order to capture the full vibration motion of a wind turbine, a measurement system is needed that fulfills the criterion of the sampling theory. Ozbek et al. (2010) made an inventory of existing vibration measurement systems for wind turbines:

\section{Accelerometer}

An accelerometer is an instrument that can measure acceleration relative to free fall (Britannica, 2010). Conceptually, an accelerometer behaves as a damped mass on a spring. When the accelerometer experiences an acceleration, the mass is displaced to the point that the spring is able to accelerate the mass. This mechanical motion is converted into an electrical signal which can be registered (Jonathan, 2010).

Accelerometers are one of the commonly used instruments to perform dynamical tests on wind turbines. However this measurement is sensitive to lightning and electromagnetic fields. It also has to be mounted on the blades of the wind turbine disturbing its aero-dynamic properties.

To obtain the positions of blades or tower, acceleration data has to be integrated twice. However, even small errors in the acceleration measured will yield a rapid increase of errors due to error accumulation (Corten and Sabel, 1995).

\section{Strain gauges}

A strain gauge is a device for measuring the changes in distances be- 
tween points in solid bodies that occur when the body is deformed (Britannica, 2010). The gauge measures the strain using a foil strain element (metal strip). If the strip of conductive metal is stretched, it will become thinner and longer, resulting in an increase of electrical resistance from end to end. Conversely, if the strip of the conductive metal is placed under compressive force, it will broaden and shorten. If these stresses are kept within the elastic limit of the metal strip, the strip can be used as a measuring element for physical force, thus the amount of applied force is inferred from the measured resistance (Sensorland, 2010).

Like accelerometers, strain gauges are also sensitive to lightning and electromagnetic fields. If strain gauges are installed within the rotation blades, they require special installation of cables for power supply and data transfer. For large commercial turbines, the required installation and preparation (sensor calibration) is very expensive and time consuming (Corten and Sabel, 1995)

\section{Fiber optic strain gauges}

Fiber optic strain gauges are composed of a multimode fiber optic cable, which is wounded in an elliptical pattern and bonded to the structure of interest. A laser is fixed within an adjustable cylindrical steel enclosure and aimed at one end of the optical fiber. The laser light emerging from the other end of the fiber forms a speckle pattern that changes as strain is applied to the structure (Figueroa et al., 2007).

These fiber optic strain gauges are a promising alternative to accelerometers and conventional strain gauges, since they are not prone to electromagnetic fields or lightning. However, this technique is still in development, and additional feasibility tests are still needed to ensure its effective and cost efficient use. Factors affecting the performance of the fiber optic sensors, such as sensitivity to temperature variations and the related error compensation are still being investigated (Rademakers et al., 2004).

\section{Laser Doppler Vibrometer}

A Laser Doppler Vibrometer (LDV) is an instrument that is used to make non-contact vibration measurements of a surface. The vibrometer is a two beam laser interferometer that measures frequency (or phase) difference between an internal reference beam and a test beam. The test beam is directed to the target, and scattered light from the target is collected and interfered with the reference beam (Kilpatrick and Markov, 2008). 
The limitation of the LDV is that vibrations can be measured only along the line of the test beam, therefore limiting the vibration measured of the wind turbine at static mode.

\section{Photogrammetry}

Photogrammetry is a measurement technique whereby $3 \mathrm{D}$ coordinates or displacements of an object can be obtained by using 2D images of the same object taken from different views. The main advantage of photogrammetry is that the images are captured remotely. Measuring points on the blades can be identified with reflective targets, which are relatively thin and do not influence the aero-dynamics of the blades. Images are able to record a relative large extent of space, such that the whole wind turbine can be measured in one image.

The challenge of this technique is to measure the relatively high frequency vibration for the blade with a high speed camera. Per frame a lot of data is obtained which is temporary stored in Random Access Memory (RAM). The transfer from RAM to permanent storage is relatively slow compared to the acquisition frequency and therefore limits the acquisition time to the available space in RAM. Another challenge lies in processing, since a large amount of computations (target measurements) have to be done in the images, before the 3D coordinates or vibration of the wind turbine can be calculated.

\subsection{Comparison of Vibration Measurement Techniques}

In order to identify the strengths and weaknesses of the described techniques to measure vibrations of a model wind turbine a comparison matrix is made. In this comparison matrix the following criteria are used that limit the operability and reliability of the measurement system:

\section{Flow field}

The flow field of the wind is directly related to the stress caused on the blade. The shape of the blades are specially designed to minimize the stress caused by the flow field. If an instrument is put on the blades, it will affect the flow field and result in incorrect measurement;

\section{Direct vibration measurement}

Direct vibration measurement is relatively difficult therefore alternative forces of the blades are measured from which the blade vibration can be calculated; 


\section{Influence of external factors}

A measuring device can be prone to external effects, for instance electrical or magnetic fields, or weather conditions creating errors in the measurements;

\section{Operation modes}

The vibration of a wind turbine can be measured in dynamic and static modes by exciting the blade with a sudden stress. The drawback of measuring at static mode is that the damping of the blade is measured, but not in operational conditions;

\section{Installation}

The rotation of a wind turbine makes it difficult to install devices in the blade that need to be connected with cables. To overcome this, slip rings or wireless connected devices can be installed.

Table 2.1: Comparison matrix of different vibration measurement techniques

\begin{tabular}{lccccc}
\hline \hline Criteria & 1 & 2 & 3 & 4 & 5 \\
\hline Accelerometer & - & - & - & + & - \\
Strain gauges & + & - & - & + & - \\
Fiber optic strain gauges & + & - & + & + & - \\
LDV & + & + & + & - & + \\
Photogrammetry & + & + & + & + & + \\
\hline
\end{tabular}

Using the five criteria, the measurement techniques described in Section 2.2 are compared to each other in Table 2.1. A negative sign is assigned to a measurement technique if it does not fulfill the criteria. From the comparison matrix it can be directly noticed that photogrammetry is the most appropriate technique to measure vibrations of a model wind turbine. Points of interest on the blades are measured by placing markers (targets), which are relatively thin and do not influence the flow field. Vibrations can be directly measured by applying 3D reconstruction with targets measured in images made from more then two views. Photogrammetry is not prone to external factors as electric or magnetic fields, it is easy to install and can operate in both static and dynamic modes. 
Chapter 2. Vibration Measurement of a Wind Turbine 


\section{Chapter 3}

\section{High Speed Photogrammetric Measurement}

From the Greek words photo (light writing) gram (graphic) metry (measure) comes the root of the science of photogrammetry. Photogrammetry is a method for image measurement and interpretation in order to derive the shape and location of an object from one or more photographs of that object (Luhmann et al., 2006). The term Close Range Photogrammetry is used when the distance to the object to be measured is less than 100 meters from the camera's position (Atkinson, 1996).

The basic steps of reconstructing an object from photographs or images are illustrated in Figure 3.1. On the left side of the figure the principal instrumentations are indicated and on the right side the methods involved. An image of an object can be correctly acquired if it is illuminated by a light source, such as the sun or any artificial light. In case of an analog camera, the reflected light from the object is recorded on film and for a digital camera it is recorded with a sensor. Objects in images can be identified and measured by their form, brightness or color distribution. For every object in an image, values can be measured in the form of radiometric data (gray value, color value) and geometric data (shape and position in the image). If an object is photographed from more than two positions, a mathematical model can be constructed on the basis of the geometrical differences caused by the shape of the object. From this mathematical model, the Three Dimensional (3D) coordinates of points on the object can be reproduced.

In Section 3.1, a more detailed description of the imaging system and its suitability for high speed applications will be given. The different target measurement techniques in digital images will be reviewed in Section 3.2. The mathematics needed to construct the 3D coordinates from images will be described in Section 3.3. 


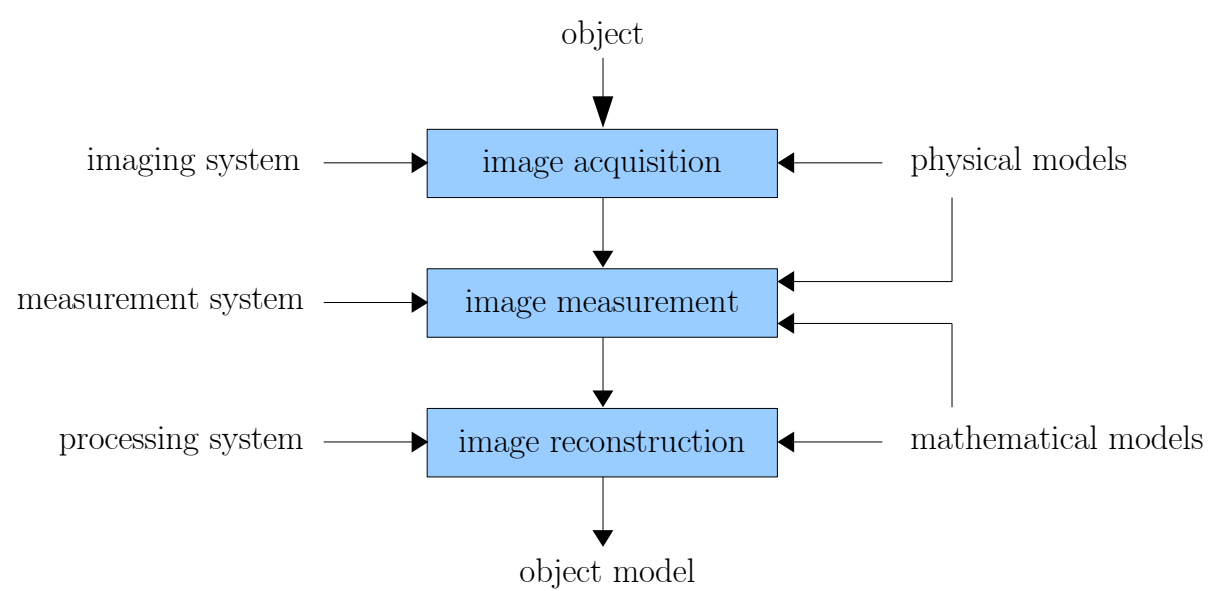

Figure 3.1: The photogrammetric process from object to model (Luhmann et al., 2006)

\subsection{High Speed Imaging}

Image acquisition is a fundamental process in photogrammetry. Images of the objects of interest must be captured and stored to allow photogrammetric measurements to be made. In general photogrammetry relies on optical processes to acquire images. Such images are typically recorded in the visible or near infrared region of the electromagnetic spectrum. The radiation is recorded by means of a perspective projection by a lens system to the plane sensor. In order to have bright and sharp images, it is important to expose the sensor to enough radiation to have a sufficient amount of energy to record. On the other hand if too much energy reaches the sensor, it will get saturated and the image will have less contrast. To control the light conditions a shutter is mounted between the lens and the sensor, which can vary in opening width and exposure time.

\subsubsection{High Speed Cameras}

To measure highly dynamical phenomena, it is important that the imaging system can acquire images faster than the deviations in the motion. That is why in high speed photogrammetry Charge-Coupled Device (CCD) chips are used, which can register (or capture) images rapidly. They also have a relatively short delay between image capture, data read and storage in RAM. CCD chips sense the light by converting photons from electromagnetic energy to electrical charge and digitizing it with an analog-to-digital converter (see 
Figure 3.2) (Atkinson, 1996).

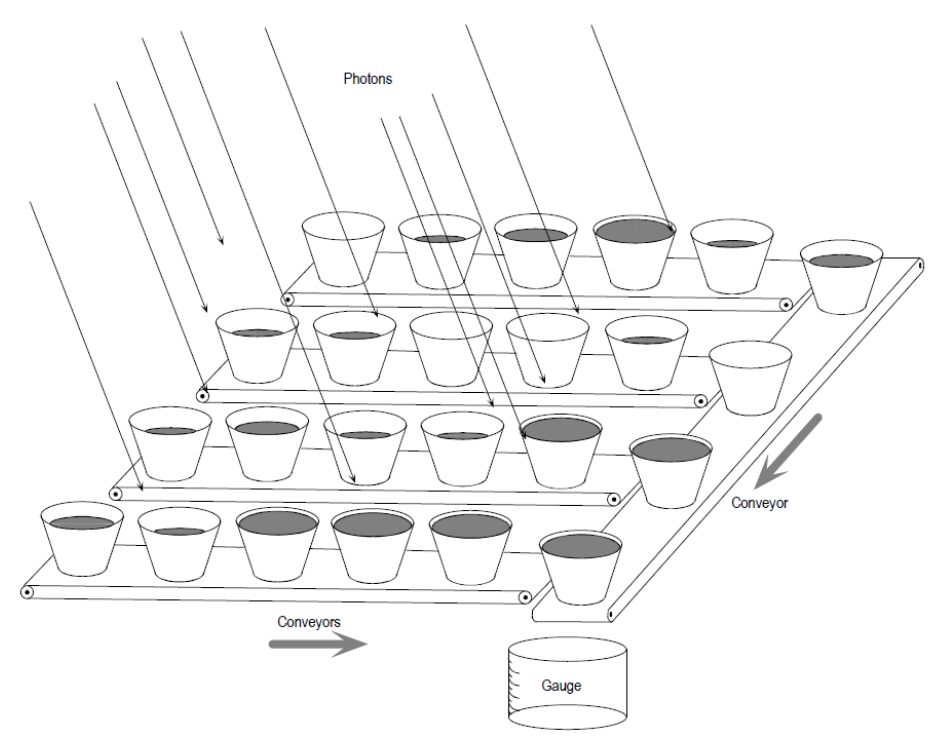

Figure 3.2: Bucket array to capture electromagnetic energy (Atkinson, 1996)

In a study of Maas (1992) he divined that a camera can be considered to be high speed, if it has an imaging rate of more then 100 fps. Further he distinguished two main types of high speed cameras. The first type are factory floor systems, which are complete systems consisting of a camera, storage device, monitor and control panel. This type of camera is used to monitor assembly lines and is not meant for geometric reconstructions. The second type is a computer based system, where the high speed camera is connected to a computer board and RAM to store the images. This system has more flexibility and can be used for geometric reconstruction.

In the manual of a Kodak high speed camera the following technical description is given (Balch, 2004). The camera has an electronic shutter and a CCD sensor able to operate at a rate of $1000 \mathrm{fps}$. The electronic shutter operates as fast as $50 \mu$ s exposure. The camera supports a monochrome or color sensor. The color sensor has a Color Filter Array which produces accurate 24-bit color. The color processing is done outside the camera when the images are transferred to a computer. This simplifies the amount of hardware in the camera and reduces the necessary data to transfer by one third. All the signal processing electronics for the CCD are within the camera's body. The camera digitizes the image data and stores it into a large memory bank. Images are then transferred to the Memory Module or through a high speed serial interface to an image server. Figure 3.3 shows a simplified block 
diagram of the camera.

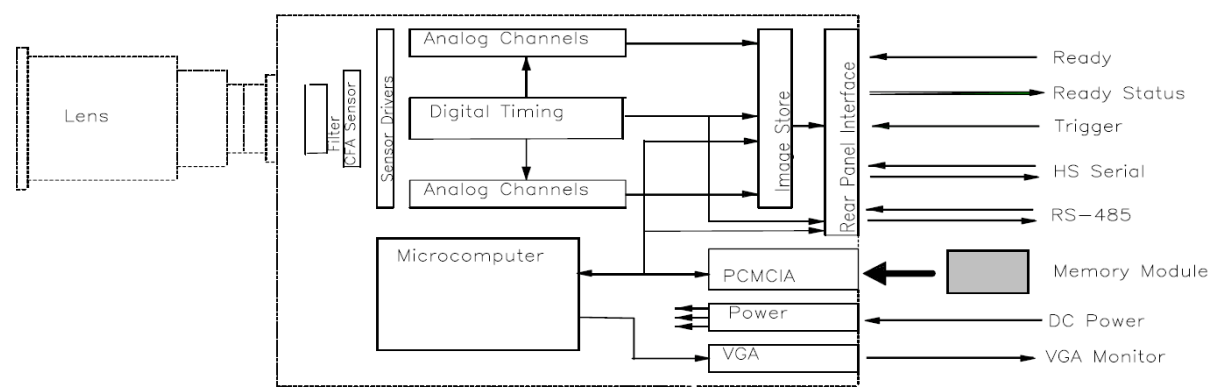

Figure 3.3: Main elements of a high speed camera (Balch, 2004)

After the images are acquired, they need to be stored on permanent memory. Since the storage process on permanent memory is relatively slow compared to the acquisition, this creates a limitation to high speed systems. Therefore it can be concluded that the amount of pictures taken in one sequence is limited by the amount of images that can be stored in the RAM of the system.

\subsubsection{Retro-Reflective Targets}

Retro-reflective targets are targets that reflect light with a strong intensity parallel to the incident direction of the incoming light. In high speed photogrammetry retro-reflective targets are used to identify objects of interest, which need to be measured. The short acquisition time (in order of $\mu \mathrm{s}$ ) used in high speed photogrammetry limits the amount of light available to get a bright and clear image. If a high speed acquisition would be made without any artificial light, the sensor would get few reflected light and the image would be dark. By using retro-reflective targets and illuminating them with a bright flash, they become visible in high speed images (see Figure 3.4).

Retro-reflective targets are made of retro-reflective materials, which are either covered by a black surrounding (mask) or are stamped in the material with a special pattern. The retro-reflective material consists of a dense arrangement of small reflective balls $(\approx 80 \mu \mathrm{m})$ or an array of micro prisms. Incident light is reflected internally within each ball or micro prism, to return parallel to its incident path (Figure 3.5). In order to achieve high contrast images, retro-reflective targets must be illuminated from the viewing direction of the camera (Luhmann et al., 2006).

Even though a target can have any size and shape, usually for retroreflective targets a circular shape is chosen. Circular shapes are ideal because 


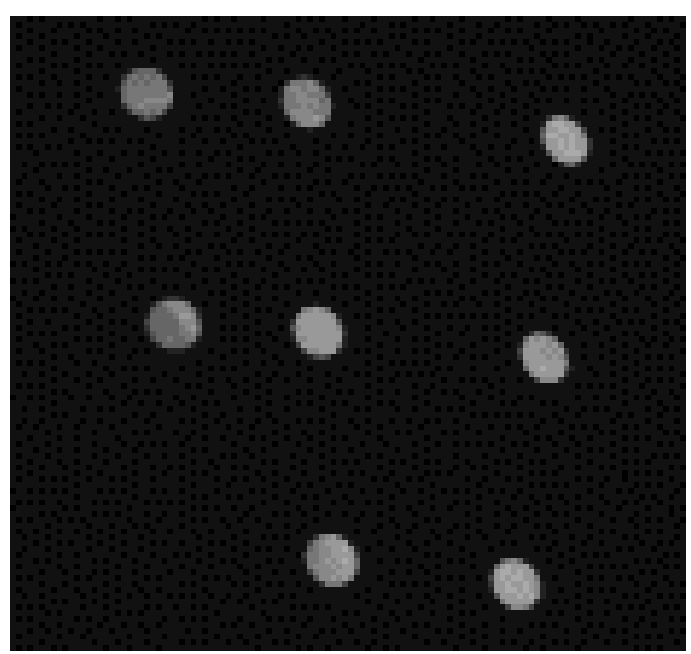

Figure 3.4: Image made with a high speed camera of circular retro-reflective targets

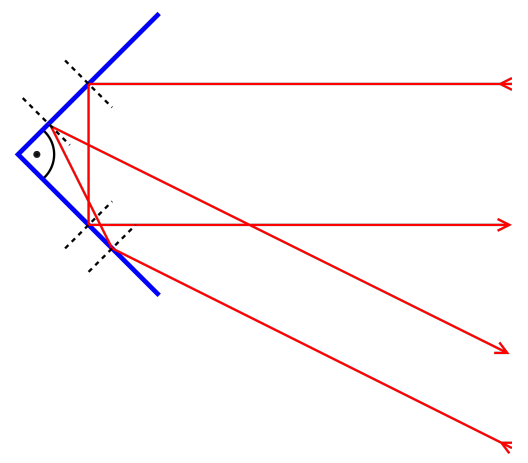

(a) Corner reflector

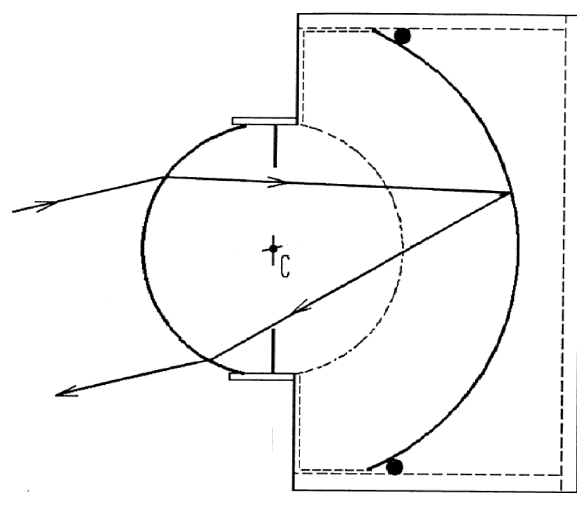

(b) Ball reflector

Figure 3.5: The principle of retro-reflective targets (Goldman, 1996) 
of their radial-symmetric design to measure the actual 3D point. Determining the target's center is rotation invariant and over a wide range of image magnifications also scale invariant.

Circular targets are not only useful for manual interaction, but also for automated digital target detection and measurement. In manual image processing, the center of the circular targets can be determined by centering a symmetric circular or point shaped marker. In digital image processing, the target center can be calculated by centroid methods, correlation with a reference pattern, or analytically by fitting a circle or ellipse (Luhmann et al., 2006).

\subsection{Automatic Target Measurement in Digi- tal Images}

In order to reconstruct an object from images, it is necessary to measure its position in two or more images made from different viewing angles. The image measurement process can be divided in two main steps, namely position measurement in individual images and establishing correspondence between the measured position in images of more views or a sequence of images. The target measurement in individual images is discussed in Section 3.2.1. In Section 3.2.2 methods are discussed to establish correspondence between measured positions in multiple images.

\subsubsection{Target Measurement in Individual Images}

In images a position can be measured of image features or targets. Image features are natural visible features of objects as homogeneous segments, boundaries, edges and other object shapes. Targets have a predefined shape as a circle or a coded pattern such that it can be clearly distinguished from other natural features. As explained in Section 3.1.2, this study uses circular retro-reflective targets and therefore the measurement of image features are out of scope and will be not discussed further. To measure circular targets in images the following techniques can be used:

\section{Template matching}

Template matching is a technique to find small specific parts of an image which match a template image or computer aided model (Kim and Arajo, 2007). Template matching is commonly used if the shape 
of the template is known, as from a circle with a known radius or from coded targets.

The basic method of template matching uses a convolution mask (containing the template) which is moved over the searched image to detect similar features in it. This technique can be easily performed on gray scale images or edge images. The convolution output will be highest at the places where the image structure matches the mask structure, since large image values get multiplied by large mask values.

This method is normally implemented by first selecting a part of the search image to use as a template. The search image can be defined as $S(x, y)$, where $(x, y)$ represent the coordinates of each pixel in the search image. The template can be defined as $T(x t, y t)$, where $(x t, y t)$ represent the coordinates of each pixel in the template. By moving the center (or the origin) of the template $T(x t, y t)$ over each $(x, y)$ point in the search image the sum is calculated of the products between the coefficients in $S(x, y)$ and $T(x t, y t)$ over the whole area spanned by the template. As all possible positions of the template with respect to the search image are considered, the positions with the highest scores are the matching positions in the search window (Kim and Arajo, 2007).

The drawback of template matching is that every position in the search image has to be considered to find matching features, making this technique computationally inefficient and therefore not suitable to process the large number of images acquired in high speed applications.

\section{Image segmentation}

Segmentation partitions an image into non-overlapping regions and boundaries, so that pixels belonging to a region have uniform gray values, while combinations of pixels in adjacent regions have significant non-uniform values. The main objective of segmentation is to reduce the amount of data to be processed and to obtain useful information about features, objects and object boundaries in the image. Another importance of segmentation is that it provides input for the next processing steps, e.g. feature recognition, matching, and image coordinates of targets to obtain exterior orientation parameters or to calculate the 3D coordinates of an object in space (Atkinson, 1996).

There are numerous methods of performing image segmentation. The most common and simplest method used is based on histogram thresholding (Atkinson, 1996). By constructing a histogram of the pixel val- 
ues, the main features, such as background and the targets can be separated from each other by a range of gray-level values centered at the peaks. In Figure 3.6 an example is given of an image with two major features respectively the coins and the background. These two features can directly be recognized by the two peaks in the histogram. The high peak belongs to the background and the second peak belongs to the coins. By assigning pixel values above a threshold to the object and the rest to the background the image is segmented.

Threshold segmentation is a simple but sufficient method to detect targets in images taken with high speed. They are taken with a short exposure time, limiting the amount of light accessing the sensor. The special reflective targets are the only features that are visible in the image, while everything else is dark and can be considered as background.

\section{Edge detection and circle fitting}

Once a target is detected and an approximate position of it is known, its center can be calculated with subpixel precision in two steps. First the edges are detected from the target based on the approximate position. Then a circle can be fitted to the edges to obtain a precise target center.

Edges are primarily image structures used for object recognition and are therefore of fundamental interest for digital image measurement and pattern recognition. Every object stands out from the background on the basis of a characteristic change in the relevant image structure. This change in image structure can be due to (Luhmann et al., 2006):

- significant changes in pixel values along physical object edges;

- changes in pixel color values (color edge);

- changes in surface texture (e.g. hatched vs. point pattern).

Besides object recognition, edges are also used to carry out precise digital image measurement or target measurement. Shortis et al. (1994) showed that once the edges of a target are detected, several methods can be applied to obtain a subpixel accurate center of the target. One of the successful methods he used, was to find a least squares fit of an arbitrary ellipse to the edges of the target.

Like for all other operations in digital image processing, there are various methods to find edges in images. The most straightforward and simple method is based on gradient changes between pixel brightness 


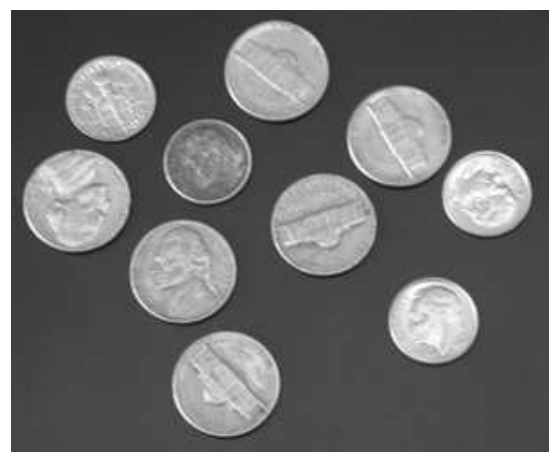

(a) Grayscale image of coins

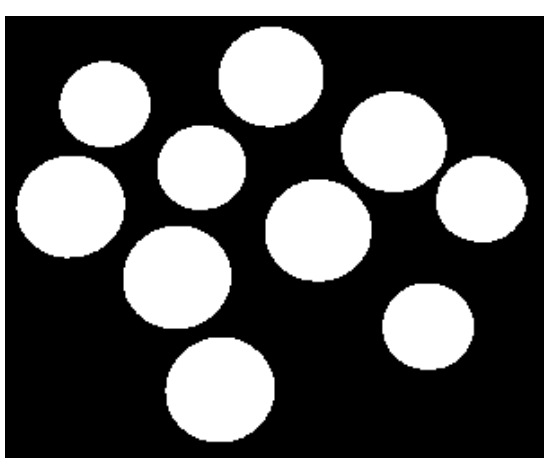

(b) Segmented image

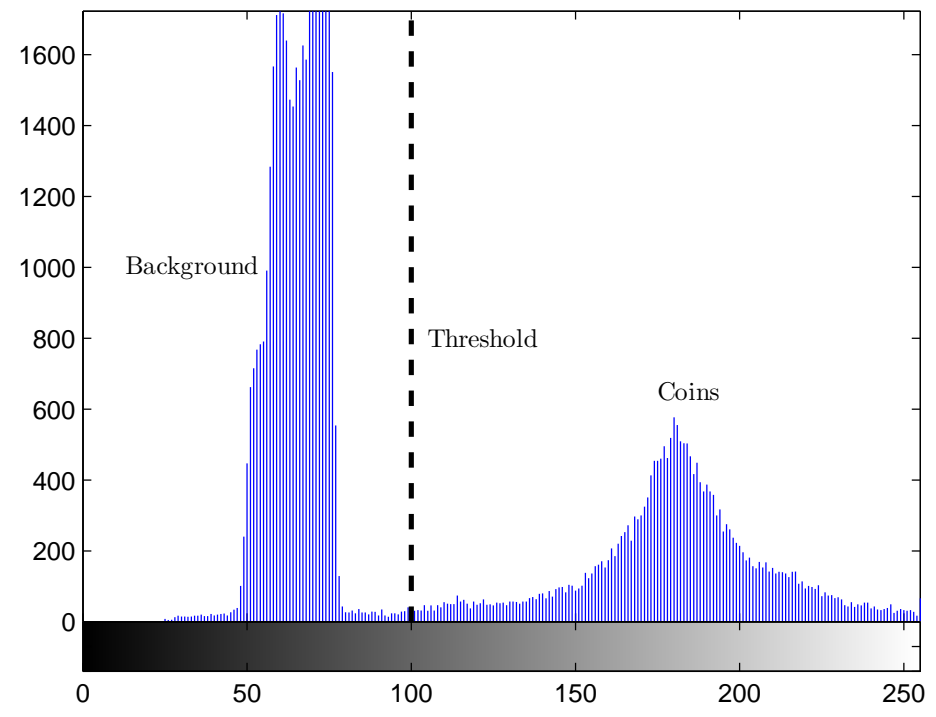

(c) Histogram of the image

Figure 3.6: Image segmented by histogram threshold 
values $(f(x, y))$ of an image. By calculating partial derivatives in the $x$ direction $\partial f / \partial x$ and the $y$ direction $\partial f / \partial y$ a gradient image can be constructed. Abrupt changes in the gradient image indicate edges of the image (Gorte, 2006).

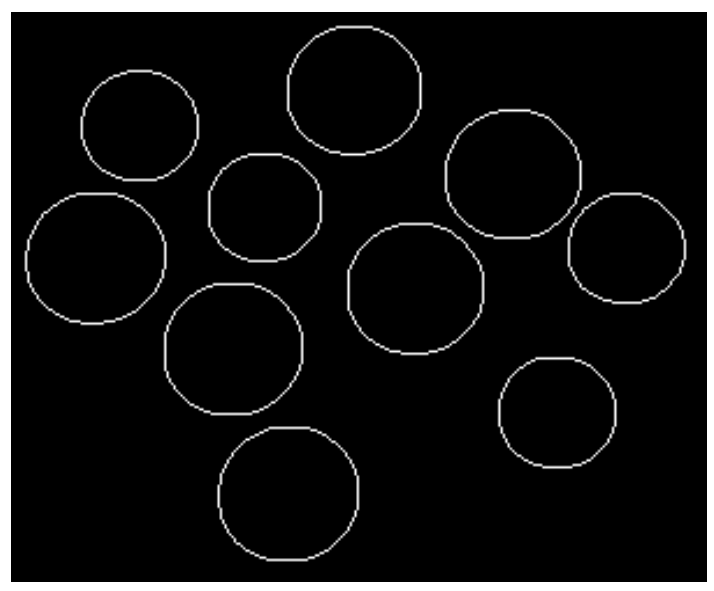

Figure 3.7: Edge detection using Canny edge detection method

A more advanced edge detection method is by Canny, which can detect edges in the presence of noise even if they are weak or not clearly visible (Figure 3.7). The Canny edge detection is performed in six steps (Green, 2002):

(a) first an image smoothing is performed to filter out any noise from the original image;

(b) then the image gradient is calculated to highlight regions with high spatial partial derivatives;

(c) from the gradient values in the $x$ and $y$ direction, an edge direction is computed;

(d) the edge direction is related to eight directions that can be traced in the image;

(e) after the edge directions are known, a non maximum suppression is applied by tracing along the edge directions and removing any pixel value that is not considered to be an edge; 
(f) at last, breaks in the edges are removed by a threshold. If the difference is below a threshold they are joined, otherwise they are disconnected.

To the detected edges a circle can be fitted to obtain the target center with subpixel precision. The circle fitting will be described in Section 4.6.4.

\subsubsection{Establishing Correspondence Between Mul- tiple Images}

Once targets have been measured in a pair or a sequence of images, the next step is to establish correspondences between them. In a pair of images establishing correspondence between targets is necessary, because the position of a target in two or more images is needed to calculate its object coordinates. In high speed applications usually two cameras are used with fixed positions. Since there are only two views containing a relatively small number of targets, it is already efficient to establish the correspondence manually.

In order to monitor the motion of an object or target, it is important to establish correspondence between the targets measured in the sequence of images. Establishing correspondences within a sequence of images has the advantage that only once correspondence has to be established between multiple views. Techniques for creating correspondence are:

\section{(a) Coded targets}

Coded targets are targets encoded with individual point identification numbers so that the point identification can be automated. The point codes are arranged in lines, rings or regions around the central target point (see Figure 3.8). In practice, several coded point identification numbers can be designed which should meet the following requirements (Luhmann et al., 2006):

- invariance with respect to position, rotation and size;

- invariance with respect to position or affine distortion;

- robust decoding;

- precisely defined and identifiable center;

- sufficient number of different point identification numbers;

- fast processing times for pattern recognition. 
The point identification is decoded by image analysis of the number and configuration of the code pattern. The pattern displayed in Figure 3.8(a) is based on barcode techniques, where the code can be reconstructed from a series of black and white marks. The pattern displayed in Figure 3.8(b) consists of additional points arranged in a local coordinate system where the target identification number is a function of the local distribution of points.

Coded targets can be automatically recognized and identified in digital images by means of image processing techniques, such as Hough transform and template matching (Shortis et al., 2003). However, the computational effort is high, and the reliability of finding the coded target is sensitive to local image quality (Ceasar and Michaelis, 1997).

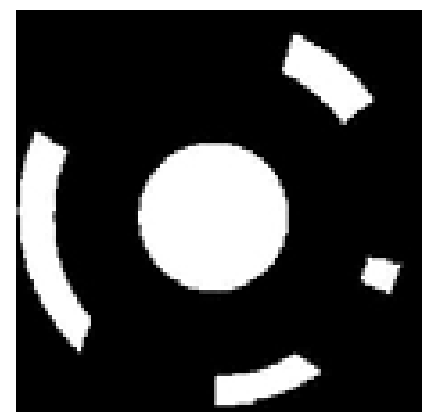

(a) Circular coded target

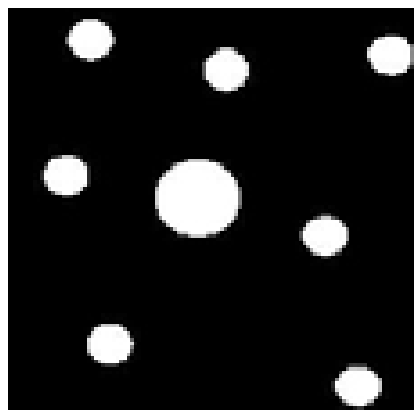

(b) Coded target with a unique pattern

Figure 3.8: An example of two coded targets (Moriyama et al., 2008)

\section{(b) Image Matching}

Image matching can be described as a method to identify and uniquely match identical object features (points, patterns, edges) in two or more images of the object (Luhmann et al., 2006).

In practice, a wide range of matching methods exist, which are grouped as area based matching, feature based matching and relational based matching (Khoshelham, 2009). Applying these techniques to find corresponding targets in a sequence of images acquired at high speed requires a method that is simple and fast to be executed. From all these methods the area based least squares matching is chosen, because this method uses a priori proximate 
location to find the match, instead of searching the whole image to find a match.

In the least squares method a target is selected by a template window and can be represented with a discrete function $f(x, y)$. The conjugate of the target in another stereo image can also be defined by a search window of the same size and represented by the function $g(x, y)$. Matching between the two windows is established if (Atkinson, 1996):

$$
f(x, y)=g(x, y)
$$

Because of random noise effects in both images a noise vector is added.

$$
f(x, y)-e(x, y)=g(x, y)
$$

By assuming that the position of the corresponding targets in both images will be relatively close to each other, which is true for images taken at high speed, the two targets can be interrelated by a translation. There might be some shear between both targets (Figure 3.9) and to model this the translation is extended to an affine translation.

$$
\begin{aligned}
& x=a_{11}+a_{12} x_{o}+a_{21} y_{o} \\
& y=b_{11}+b_{12} x_{o}+b_{21} y_{o}
\end{aligned}
$$

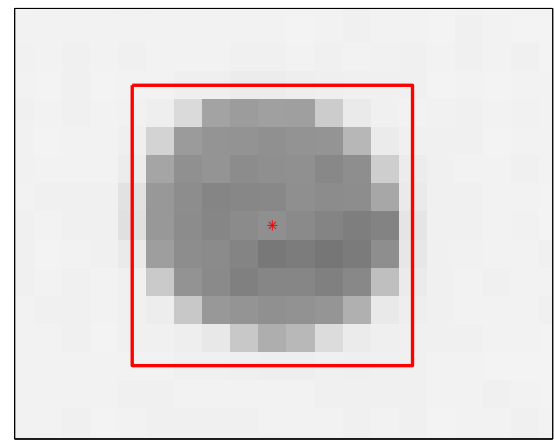

(a) Circular target

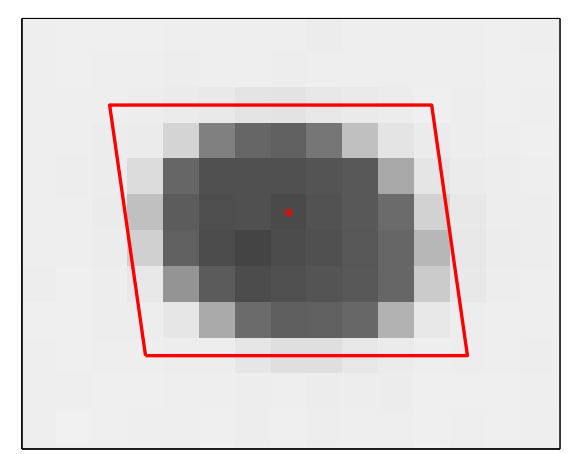

(b) Sheared circular target

Figure 3.9: Shear between matching targets

Where $a_{11}$ and $b_{b 11}$ are the transformation parameters and $a_{12}$, $a_{21}, b_{12}$ and $b_{21}$ are the affine parameters respectively in the $x$ and $y$ axis. This equation is linearized (by differentiation to $d x, d y$ ) 
and two radiometric parameters $r_{s}$ (shift) and $r_{t}$ (scale) are added to the system (see the book of Atkinson (1996) for more details), resulting in:

$$
\begin{aligned}
f(x, y)-e(x, y)= & g^{o}(x, y)+g_{x} d a_{11}+g_{x} x_{o} d a_{12}+ \\
& g_{x} y_{o} d a_{21}+g_{y} d b_{11}+g_{y} x_{o} d b_{12}+ \\
& g_{y} y_{o} d b_{21}+r_{s}+g^{o}(x, y) r_{t}
\end{aligned}
$$

from which the unknown parameters are:

$$
x^{T}=\left\{d a_{11}, d a_{12}, d a_{21}, d b_{11}, d b_{12}, d b_{21}, r_{s}, r_{t}\right\}
$$

Using Equation 3.4, a set of equations can be constructed between pixels of the template and search window to solve the affine parameters. The unknown parameters can be calculated by means of a least squares estimation. With the obtained affine parameters, the position of the targets in the search image can be refined. The quality of the affine parameters is defined by the residuals of the least squares estimation. As the quality in the first least squares estimation may not be satisfying due to an imperfect match, the whole estimation process can be repeated until a desired quality of match is achieved (Figure 3.10).

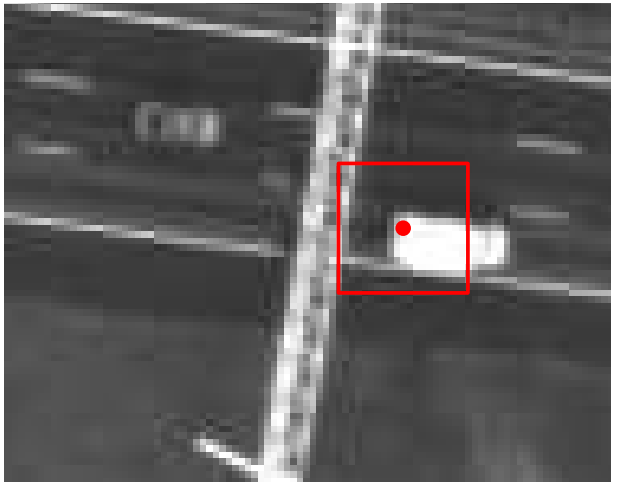

(a) Feature selected in reference image

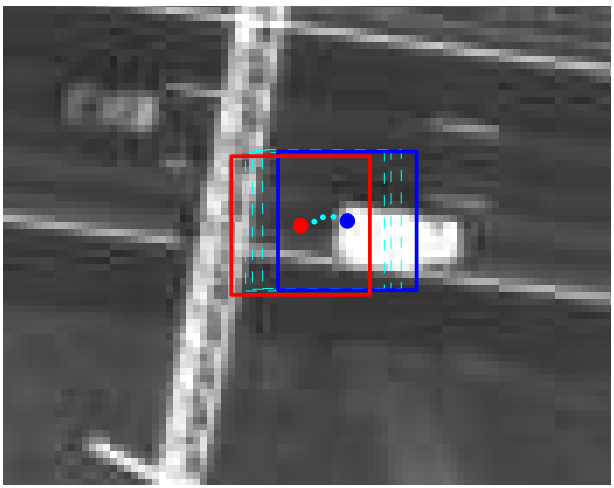

(b) Match in the search image

Figure 3.10: Least squares matching between two overlapping images

The drawback of least squares image matching is that the match does not necessarily converge to a correct solution, but it can diverge or even stays oscillating between two or more wrong solutions. In Figure 3.11 an example is given where least squares 
matching is applied on a high speed image. The blue dot is the approximated position of the target in the search image, the dots in magenta are the positions calculated in the iteration, the red dot is the match that was found and the green dot is the correct position in the image. It can be noticed that the match did not converge to the correct solution which can be explained as follows:

- the targets are representative patterns, and are therefore indistinguishable from each other;

- the background has no texture which can lead the match to the correct solution. Instead the background is random, which also explains the random path of the matches.

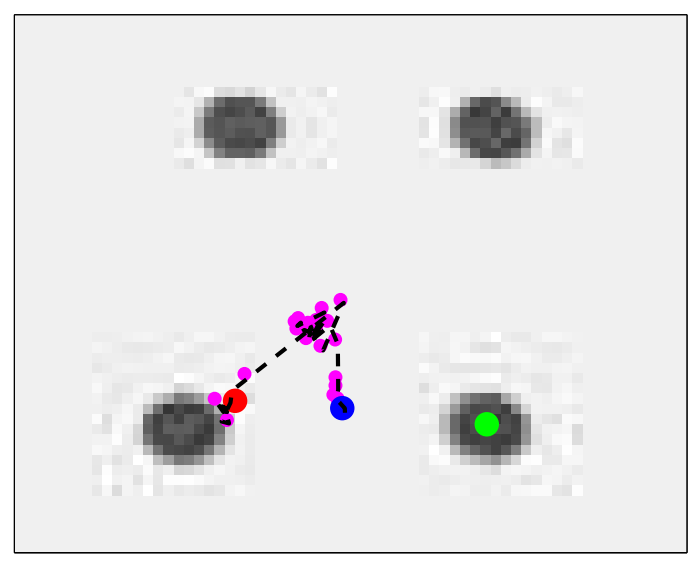

Figure 3.11: Example of least squares image matching applied on high speed images

\section{(c) Target Tracking}

The reason for using high speed imagery in most applications is to capture dynamical motion on a fast and frequent base so that it can be completely reconstructed. This knowledge of a minimum motion between a sequence of images can also be used to find an approximate location of the corresponding targets. Tracking can also be achieved by fitting a mathematical model to the motion detected in the first frames and predicting the position in the next frames. The prediction or approximate location can then be further refined by image matching or with edge detection and circle fitting. By the use of a mathematical model in the tracking, 
this method is not prone to representative patterns of the targets in high speed images.

In a study by Ariyawansa and Clarke (1997) about high speed correspondence of object recognition and tracking, they demonstrated that target tracking in a sequence of images is relatively simple compared to directly finding the match. It saves processing time, making the whole process faster. An example of human motion tracking is illustrated in Figure 3.12, where the correspondence was created using a mathematical constraint assuring that the acceleration (second derivative) of a moving human body may have no discontinuities.

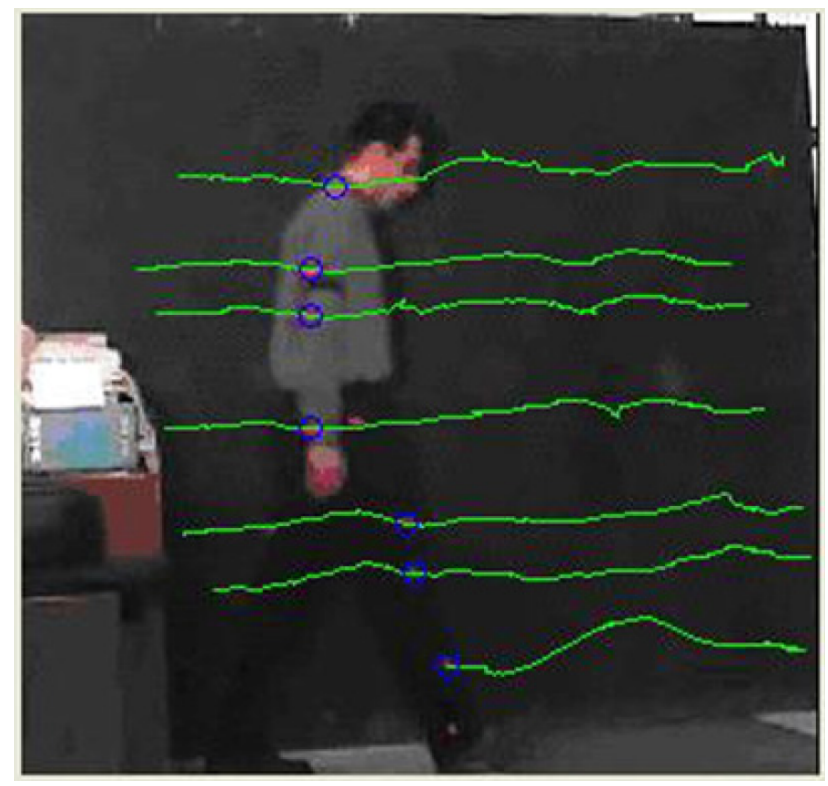

Figure 3.12: Tracking of human motion (Kolahi et al., 2007)

\subsection{Mathematical Fundamentals of Pho- togrammetry}

The position of a point in object space is defined by three coordinates. However, in images this is reduced to a two dimensional space while information is lost in one dimension. By making images of the object from two or more viewing positions, the third dimension can be reconstructed. The relative change between the position of the camera and 
the object causes geometric changes to the shape of the object in the image. By mathematically modeling the geometric changes, the central perspective imaging system and optical distortions the object can be reconstructed in 3D (Luhmann et al., 2006).

In Section 3.3.1 a brief overview will be given of the different coordinate systems involved in photogrammetry. These coordinate systems are related to each other with mathematical transformations and are called orientations. The interior orientation of the camera is explained in Section 3.3.2, while the exterior orientation is explained in Section 3.3.3. The mathematical equation relating the object points and the image points is described in Section 3.3.4 and Section 3.3.5. The 3D object reconstruction from image coordinates is described in Section 3.3.6.

\subsubsection{Coordinate Systems}

In photogrammetry there are multiple coordinate systems in which measurements are made. These spaces need to be interconnected to obtain reliable $3 \mathrm{D}$ information. The coordinate systems are as follows (Khoshelham, 2009):

\section{- Pixel coordinate system}

this quantifies the image in a two dimensional coordinate system defined by the rows and columns of the matrix sensor. The origin is usually chosen at the center of the image (see Figure 3.13(a));

\section{- Camera coordinate system}

is a $3 \mathrm{D}$ coordinate system defined by the perspective center of the camera. The perspective center plays a key role in the mathematical model to relate points in the image to its correspondence in object space. The origin of this system is defined at the optical center of the camera. The $x$ and $y$ axis are parallel to the image sensor and the $z$ axis approximately coincides with the optical axis (see Figure 3.13(b));

\section{- Model coordinate system}

is a $3 \mathrm{D}$ system that describes the relative position and orientation between two or more images. Normally its origin is chosen at the perspective center of one of the images. In addition the $x$ and $y$ axis can be parallel to the related camera coordinate system (see Figure 3.13(c)); 
- Object coordinate system

this can be any global or local reference cartesian coordinate system $(X, Y, Z)$ defined by reference points on the object. For example national geodetic coordinate systems are defined by geodetically measured reference points. An example of a local system is a workpiece system of the body of a car defined by its constructional chassis (see Figure 3.13(d)).

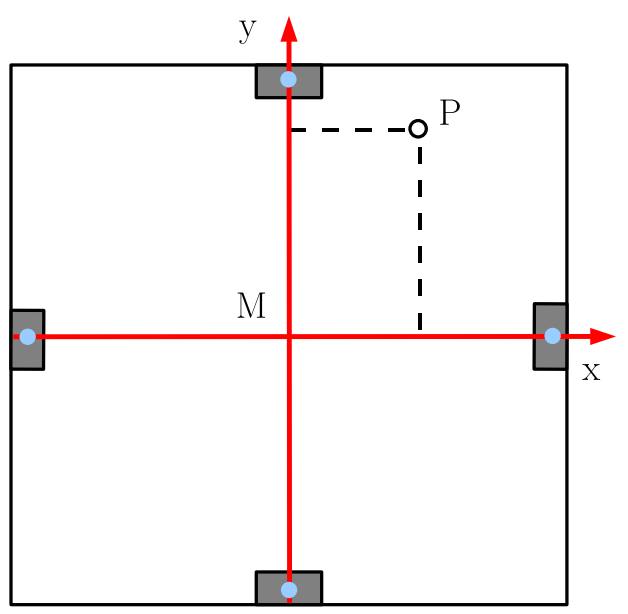

(a) Pixel image coordinate systems

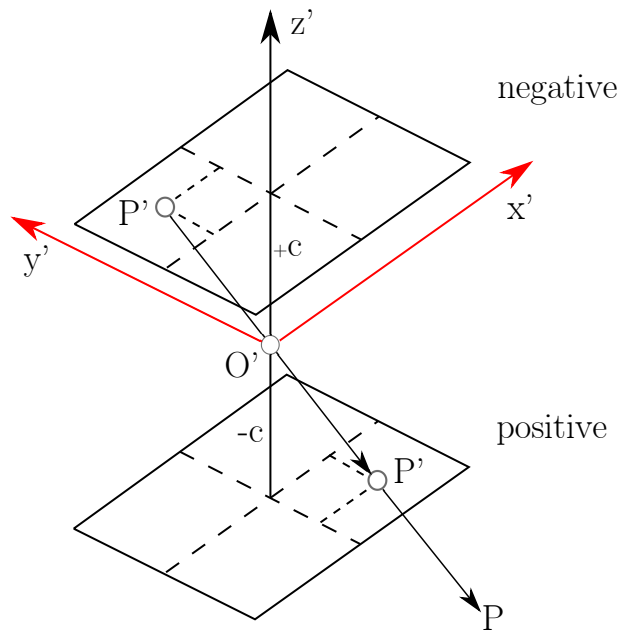

(b) Camera coordinate systems

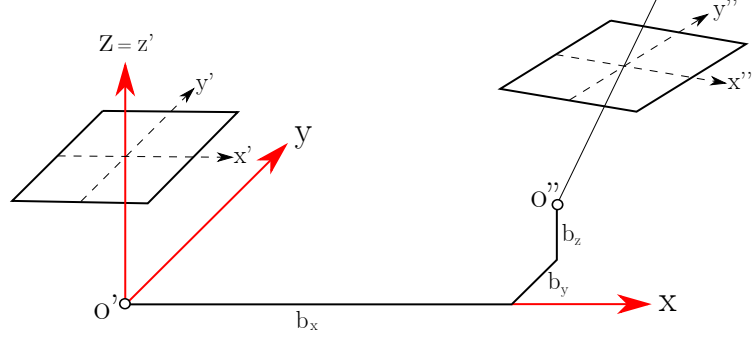

(c) Model coordinate system

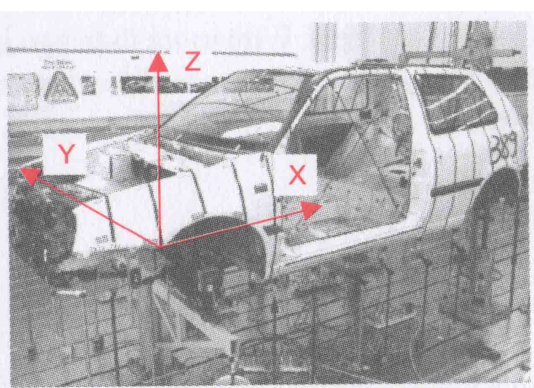

(d) Object coordinate system

Figure 3.13: The Coordinates systems used in photogrammetry (Luhmann et al., 2006)

In order to reconstruct objects measured in images, these coordinate systems need to be related to each other by mathematical transformations. The pixel coordinate system can be related to the camera coordinate system by an interior orientation process. In the interior 
orientation, the internal geometry of the camera is reconstructed by aligning the image plane to the perspective center of the lenses. The lenses also cause distortions in the image, which are also corrected in the interior orientation. In Section 3.3.2 a more detailed description is given of the interior orientation.

The camera coordinate system and the object coordinate system are related to each other by an exterior orientation process. In this process, the position and orientation of the origin of the perspective center is determined in the object coordinate system. Once the position and orientation of the perspective center are determined, the object coordinates of image points can be calculated using a projective transformation defined by the collinearity conditions. Usually the exterior orientation is performed in two steps namely relative orientation and absolute orientation. In relative orientation, a 3D model of an object is reconstructed in an arbitrary scale by orienting one image with respect to its stereo pair. The obtained relative model in arbitrary scale can then be transformed to the object coordinate system by an absolute orientation process. In Section 3.3.3 a more detailed description will be given of the exterior orientation.

The mathematics of all the orientation steps are based on the collinearity equation and will be described in Section 3.3.4. Since the collinearity equations are nonlinear an alternative linear method called DLT is described in Section 3.3.5 to obtain the interior and exterior orientation.

\subsubsection{Interior Orientation}

The interior of a camera can be modeled as a spatial system containing a planar imaging area (film or electronic sensor) and a lens with a perspective center $O^{\prime}$. In the theoretical model of the perspective projection, the optical perspective axis $O^{\prime} H^{\prime}$ coincides with the center of the pixel coordinate $m^{\prime}$. However, in reality there is an offset between the image center and the intersection of the optical axis with the image plane, as illustrated in Figure 3.14 (Luhmann et al., 2006).

The parameters of the interior orientation of a camera define the spatial position of the perspective center, the principal distance and the location of the principal point. They also include deviations caused by lens imperfections such as radial and tangential lens distortions and image affinity and orthogonality. The interior orientation consists of the following parameters (Khoshelham, 2009): 


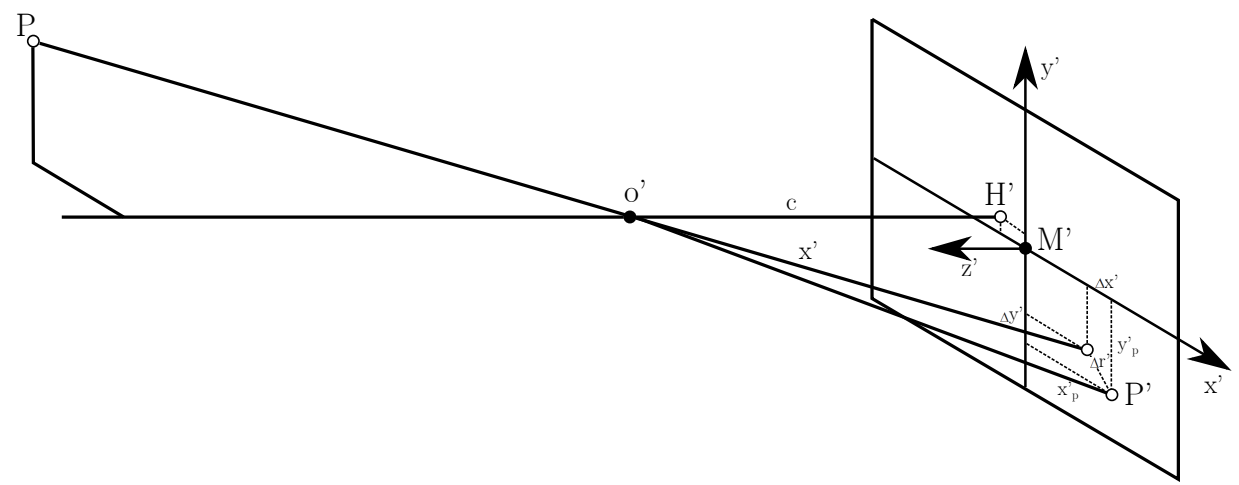

Figure 3.14: Interior geometry of a camera (Luhmann et al., 2006)

\section{- Focal length}

to correct the $z$ coordinate or the distance from the perspective center to the image center;

\section{- Principal point offset}

to correct for the deviation between the center of the image plane and the principal point. The correction is applied by a $2 \mathrm{D}$ translation and in case of a shear angle between the axes, a 2D affine transformation is applied;

\section{- Lens distortions}

to rectify radial and tangential distortions;

- Pixel size

to transform the discrete pixel coordinates to continuous coordinates.

If all these parameters are known, the error free imaging factor $x^{\prime}$ in Figure 3.14 can be defined with respect to the perspective center using the equation:

$$
x^{\prime}=\left[\begin{array}{l}
x^{\prime} \\
y^{\prime} \\
z^{\prime}
\end{array}\right]=\left[\begin{array}{ccc}
x_{p}^{\prime} & -x_{0}^{\prime} & -\Delta x^{\prime} \\
y_{p}^{\prime} & -y_{0}^{\prime} & -\Delta y^{\prime} \\
& -c &
\end{array}\right]
$$

$x_{p}^{\prime}, y_{p}^{\prime}$ : measured coordinates of image point $P^{\prime}$

$x_{0}^{\prime}, y_{0}^{\prime}$ : coordinates of the principal point $H^{\prime}$

$\Delta x^{\prime}, \Delta y^{\prime}:$ axis related correction lens distortions 
All interior orientation parameters are estimated in a camera calibration process. This is done by obtaining several images of a pattern of points, with known positions in object space. Then, the image coordinates of the pattern points are measured in all images. The image and object coordinates of the pattern are put together with the other calibration parameters in a mathematical model, the collinearity equations (Equation 3.19, which will be discussed in Section 3.3.4). For more background information about the camera calibration see the book of Luhmann et al. (2006).

\subsubsection{Exterior Orientation}

After the interior geometry of an image is reconstructed, the next step is to construct the exterior geometry by locating the position of the camera coordinate system in object space and relative to other images (Figure 3.15). The exterior orientation consists of six parameters which describe the spatial positioning and orientation of the camera coordinate system with respect to the global coordinate system.

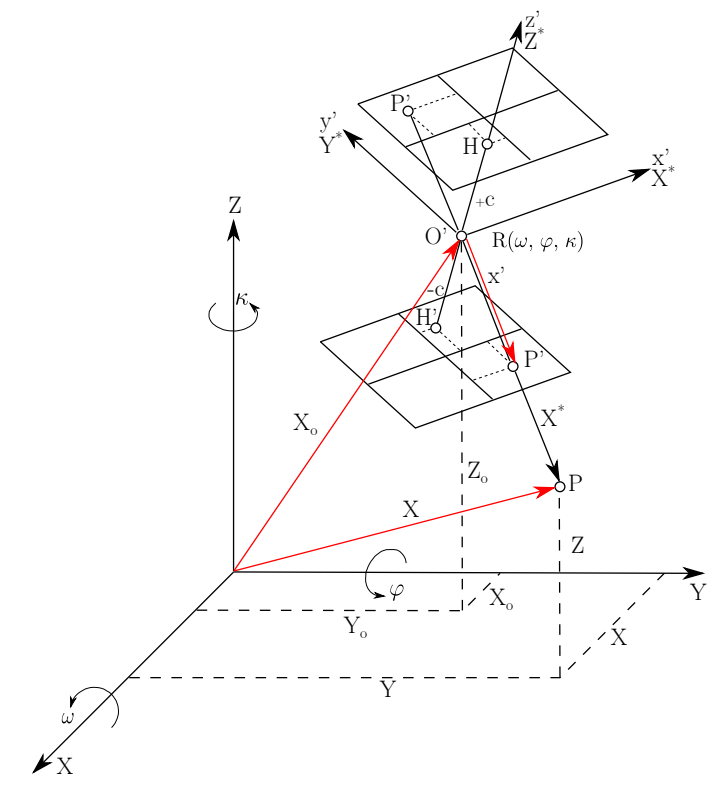

Figure 3.15: External orientation of a camera (Luhmann et al., 2006)

The spatial position of the camera coordinate system is defined by the translation vector $X_{o}$ from the origin in space to the perspective center 
$O^{\prime}$. The angular orientation in space is defined by three independent rotations $\omega, \varphi, \kappa$ about the axes $X, Y, Z$ respectively. The elements of the rotation matrix can be defined as a trigonometric function of the rotational angles.

$$
\begin{gathered}
X_{0}=\left[\begin{array}{l}
X_{0} \\
Y_{0} \\
Z_{0}
\end{array}\right]: \text { translation vector } \\
R=\left[\begin{array}{lll}
r_{11} & r_{12} & r_{13} \\
r_{21} & r_{22} & r_{23} \\
r_{31} & r_{32} & r_{33}
\end{array}\right] \text { : rotation matrix } \\
R=\left[\begin{array}{ccc}
\cos (\varphi) \cos (\kappa)-\sin (\varphi) \sin (\omega) \sin (\varphi) & -\sin (\varphi) \cos (\omega) & \cos (\varphi) \sin (\kappa)+\sin (\varphi) \cos (\kappa) \\
\sin (\varphi) \sin (\kappa)-\cos (\varphi) \cos (\omega) \cos (\varphi) & -\cos (\varphi) \sin (\omega) & \sin (\varphi) \cos (\kappa)+\cos (\varphi) \sin (\kappa) \\
-\cos (\omega) \sin (\kappa) & \sin (\omega) & \cos (\omega) \cos (\kappa)
\end{array}\right]
\end{gathered}
$$

With the given parameters of the exterior orientation, the vector from the perspective center $O^{\prime}$ to the image point $P^{\prime}$ (image vector $x^{\prime}$ ) can be transformed into an absolute oriented spatial ray from the perspective center to the object point $P$. For a single image, the exterior orientation parameters can be computed if object coordinates of at least three points are known, which do not lie on a straight line. This method is called space resection(Luhmann et al., 2006). The exterior orientation can also be performed in two steps, namely relative and absolute orientation.

\section{Relative Orientation}

The relative orientation is performed on a pair of stereo images of an object. The images are relatively oriented with respect to each other, from which a relative 3D model of the object can be constructed.

The relative orientation describes the translation and rotation of one image with respect to its stereo pair in a common local model coordinate system. The numerical model of the relative orientation is defined by a local three dimensional coordinate system $x y z$ located in the perspective center of the first (left) image and oriented parallel to its image coordinate system (see Figure 3.16). The parameters of the exterior orientation of the left image with respect to the model coordinate system 
are therefore:

$$
\begin{array}{ll}
x_{1}=0 & \omega_{1}=0 \\
y_{1}=0 & \varphi_{1}=0 \\
z_{1}=0 & \kappa_{1}=0
\end{array}
$$

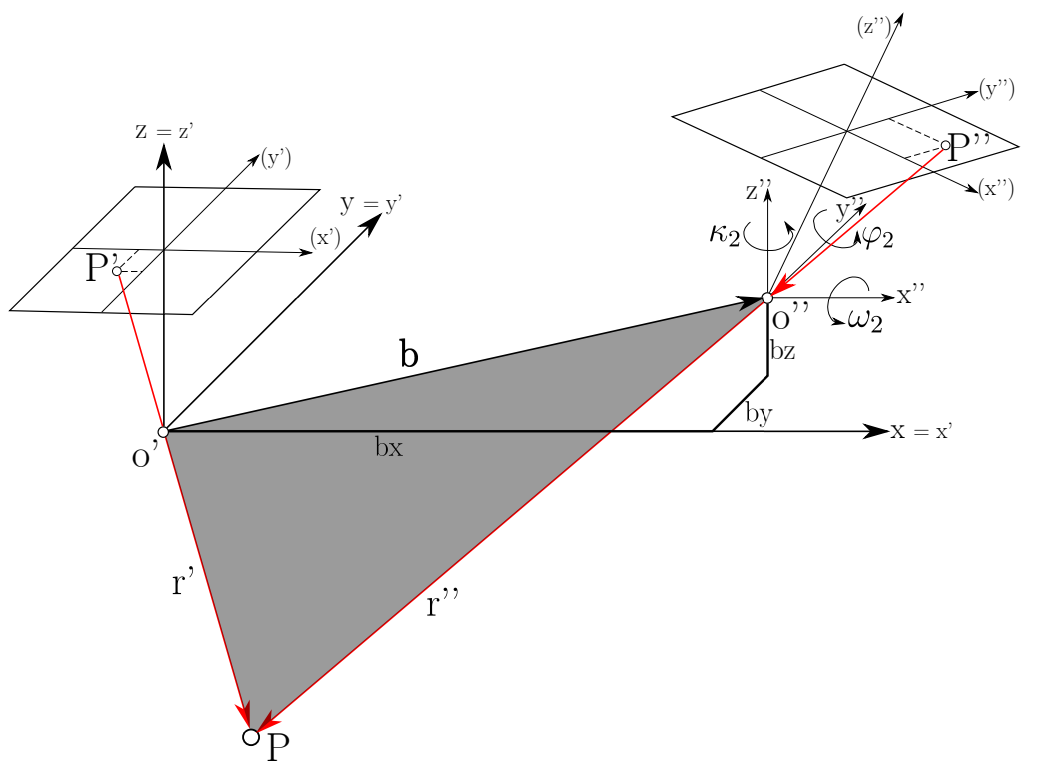

Figure 3.16: Relative orientation of the two stereo images (Luhmann et al., 2006)

The second (right) image is oriented in the model coordinate system by three translations and three rotations:

$$
\begin{array}{ll}
x_{2}=b x & \omega_{2} \\
y_{2}=b y & \varphi_{2} \\
z_{2}=b z & \kappa_{2}
\end{array}
$$

The base space vector $\mathbf{b}$ between the perspective centers $O^{\prime}$ and $O^{\prime \prime}$ is defined by the base components $b x, b y$ and $b z$. The relative orientation parameters can be calculated using the coplanarity constraint. This constraint implies that an object point $P$ imaged to $P^{\prime} P^{\prime \prime}$, the vectors $r^{\prime}, r^{\prime \prime}$ and $b$ are coplanar and lie in the epipolar plane of the target $P$ and the two perspective centers $O^{\prime}$ and $O^{\prime \prime}$. Relative to the primary 
axis these vectors are:

$$
\begin{aligned}
a_{1} & =-\left[\begin{array}{llll}
x_{1} & y_{1} & -c_{1}
\end{array}\right]^{t} \\
a_{2} & =-R^{t}\left[\begin{array}{cccc}
x_{2} & y_{2} & -c_{2}
\end{array}\right]^{t} \\
\mathbf{b} & =
\end{aligned}
$$

where $R$ is the rotational matrix. The triple scalar product of the three coplanar vectors is zero, so $b a_{1} \times a_{2}=0$, or

$$
\operatorname{det}\left[\begin{array}{ccc}
b_{x} & x_{1} & r_{11} x_{2}+r_{11} y_{2}-r_{31} c_{2} \\
b_{y} & y_{1} & r_{12} x_{2}+r_{12} y_{2}-r_{32} c_{2} \\
b_{z} & z_{1} & r_{13} x_{2}+r_{13} y_{2}-r_{33} c_{2}
\end{array}\right]=0
$$

Assuming that at least one of the components (say $b_{x}$ ) is nonzero and putting $R^{t} a_{2}=a_{2}^{\prime}=\left[\begin{array}{lll}x_{2}^{\prime} & y_{2}^{\prime} & z_{2}^{\prime}\end{array}\right]^{t}$ :

$$
\operatorname{det}\left[\begin{array}{ccc}
1 & x_{1} & x_{2}^{\prime} \\
\frac{b_{y}}{b_{x}} & y_{1} & y_{2}^{\prime} \\
\frac{b_{z}}{b_{x}} & -c_{1} & z_{2}^{\prime}
\end{array}\right]=0
$$

This equation is the coplanarity equation of the target $P$. There are five elements in the relative orientation that need to be solved: $\left(b_{y} / b_{x}\right)$, $\left(b_{z} / b_{x}\right), \omega, \varphi$ and $\kappa$. Three image coordinates well distributed over the object space are needed to evaluate the parameters of the relative orientation. To optimize the quality of the solution, it is recommended to use more than the required minimum and evaluate it with a least squares estimation. For more details about relative orientation, the books of Atkinson (1996) and Luhmann et al. (2006) are recommended.

Using the obtained relative orientation parameters and image coordinates of a target measured in the two stereo images, 3D coordinates can be calculated relative to the $\left(x_{1}, y_{1}, z_{1}\right)$ axes. These coordinates are referred to as model coordinates.

\section{Absolute Orientation}

Absolute orientation describes the transformation of the model coordinates $x y z$ obtained from a relative orientation with an arbitrary position, rotation and scale, into the object coordinate system $X Y Z$ via reference points (Luhmann et al., 2006). Reference points are object 
points measured in the model space and have known coordinates in the object coordinate system.

Mathematically, the absolute transformation is described as a spatial similarity transformation with three translations, three rotations and one scaling factor. The transformation is given by the equation (Luhmann et al., 2006):

$$
\left[\begin{array}{l}
X \\
Y \\
Z
\end{array}\right]=\left[\begin{array}{c}
X_{m} \\
Y_{m} \\
Z_{m}
\end{array}\right]+m\left[\begin{array}{lll}
r_{11} & r_{12} & r_{13} \\
r_{21} & r_{22} & r_{23} \\
r_{31} & r_{32} & r_{33}
\end{array}\right]
$$

where $m$ is the scale.

To solve the parameters of the transformation, a minimum of seven suitable point elements are required, which can be obtained from three spatially well distributed $X Y Z$ reference points. Figure 3.17 gives an illustration of the transformation of the model coordinate system $x y z$ to the object coordinate system $X Y Z$.

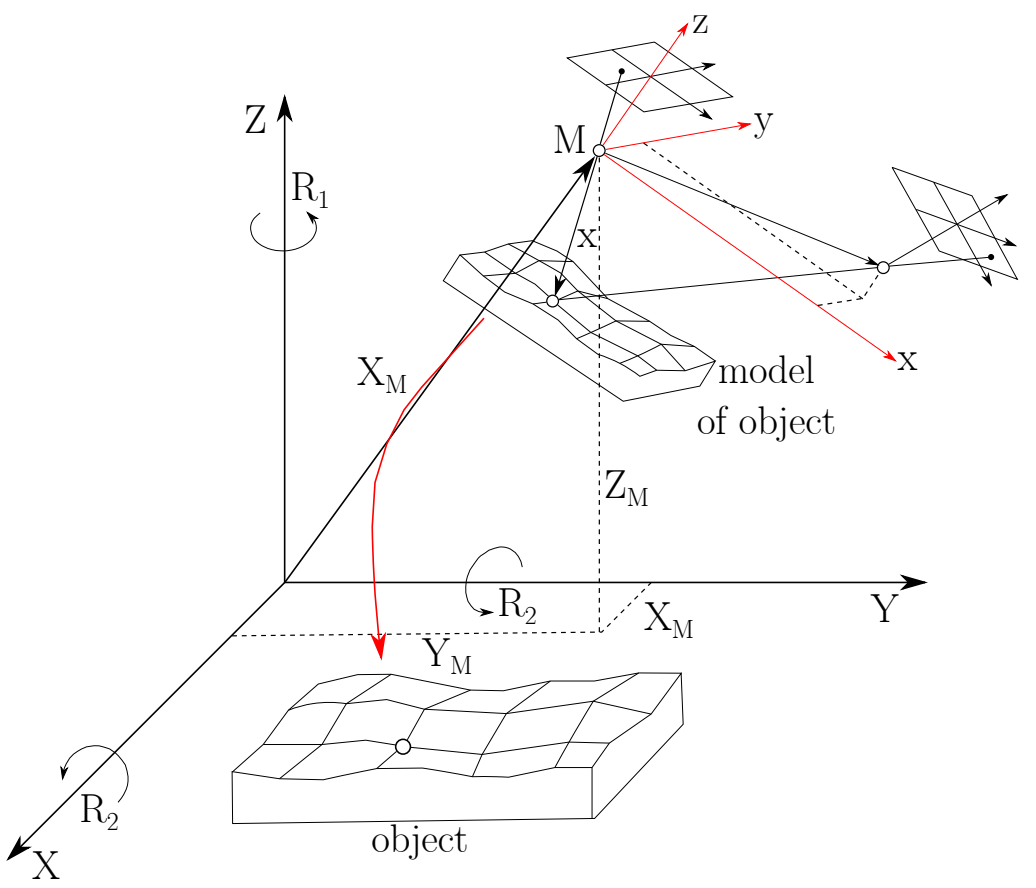

Figure 3.17: Absolute orientation of a model coordinate system to the object coordinate system (Luhmann et al., 2006) 


\subsubsection{Collinearity Equations}

The previously described orientation steps are based on the central perspective projection of the imaging system. This implies that a point in object space, its projection in the image and the perspective center all lie on one line or are collinear. Thus coordinates $(\mathbf{X})$ of the object point $P$ from Figure 3.15 can be derived from the principal point offset vector $X_{o}$ and the vector $X^{*}$ from the perspective center to the object point.

$$
\mathbf{X}=X_{0}+X^{*}
$$

The vector $X^{*}$ is given in the object coordinate system. The image vector $\mathrm{x}^{\prime}$ may be transformed into object space by a rotation matrix $R$ and scaled by a factor $m$.

$$
X^{*}=m R x^{\prime}
$$

The projection of an image point into a corresponding object point can be formulated as:

$$
\begin{gathered}
\mathbf{X}=X_{0}+m R x^{\prime} \\
{\left[\begin{array}{c}
X \\
Y \\
Z
\end{array}\right]=\left[\begin{array}{c}
X_{0} \\
Y_{0} \\
Z_{0}
\end{array}\right]+m\left[\begin{array}{lll}
r_{11} & r_{12} & r_{13} \\
r_{21} & r_{22} & r_{23} \\
r_{31} & r_{32} & r_{33}
\end{array}\right]\left[\begin{array}{c}
x^{\prime} \\
y^{\prime} \\
z^{\prime}
\end{array}\right]}
\end{gathered}
$$

The scale factor $m$ is an unknown value which varies for each object point. If only one image is available, then only the direction to an object point $P$ can be determined but not the absolute position in space. The 3D coordinates of $P$ can be computed if this spatial direction intersects another geometrical known element (e.g. intersection with a second ray from another image). By inverting the Equation 3.17, adding the principal point offset $H^{\prime}\left(x^{\prime}{ }_{o}, y^{\prime}{ }_{o}\right)$ and introducing the correction terms $\delta x^{\prime}$ (image distortion parameters), the image coordinates are given by:

$$
\begin{gathered}
x^{\prime}-x^{\prime}{ }_{o}-\Delta x^{\prime}=\frac{1}{m} R^{-1}\left(X-X_{o}\right) \\
{\left[\begin{array}{ccc}
x^{\prime} & x^{\prime}{ }_{0} & -\Delta x^{\prime} \\
y^{\prime} & y_{0}^{\prime} & -\Delta y^{\prime} \\
& z^{\prime} &
\end{array}\right]=\frac{1}{m}\left[\begin{array}{lll}
r_{11} & r_{12} & r_{13} \\
r_{21} & r_{22} & r_{23} \\
r_{31} & r_{32} & r_{33}
\end{array}\right]\left[\begin{array}{c}
X-X_{0} \\
Y-Y_{0} \\
Z-Z_{0}
\end{array}\right]}
\end{gathered}
$$


By dividing the first and the second equations by the third equation, the unknown scaling factor $m$ is eliminated and the collinearity equations are obtained:

$$
\begin{aligned}
& x^{\prime}=x^{\prime}{ }_{o}+z^{\prime} \frac{r_{11}\left(X-X_{0}\right)+r_{21}\left(Y-Y_{0}\right)+r_{31}\left(Z-Z_{0}\right)}{r_{13}\left(X-X_{0}\right)+r_{23}\left(Y-Y_{0}\right)+r_{33}\left(Z-Z_{0}\right)}+\Delta x^{\prime} \\
& y^{\prime}=y_{0}^{\prime}+z^{\prime} \frac{r_{12}\left(X-X_{0}\right)+r_{22}\left(Y-Y_{0}\right)+r_{32}\left(Z-Z_{0}\right)}{r_{13}\left(X-X_{0}\right)+r_{23}\left(Y-Y_{0}\right)+r_{33}\left(Z-Z_{0}\right)}+\Delta y^{\prime}
\end{aligned}
$$

These equations describe the transformation of object coordinates $(X, Y, Z)$ into corresponding image coordinates $\left(x^{\prime}, y^{\prime}\right)$ as a function of the interior orientation parameters $\left(x_{0}^{\prime}, y_{0}^{\prime}, c, \Delta x^{\prime}, \Delta y^{\prime}\right)$ and the exterior orientation parameters $\left(X_{0}, Y_{0}, Z_{0}, \omega, \varphi, \kappa\right)$.

The drawback of collinearity equations is, that they are nonlinear and therefore cannot be solved with standards LSQ adjustment. To solve the unknown parameters the system of equations have to be linearized and initial values are needed.

\subsubsection{Direct Linear Transformation}

An alternative method of calculating a transformation between image coordinates and object coordinates is the Direct Linear Transformation (DLT). This method is commonly used in machine vision and was developed by Karara and Abdel-Aziz (1971). This method is based on the collinearity equations, extended by an affine transformation of the image coordinates. The transformation equations of the DLT are as follows:

$$
\begin{aligned}
& x=\frac{L_{1} X+L_{2} Y+L_{3} Z+L_{4}}{L_{9} X+L_{10} Y+L_{11} Z+1} \\
& y=\frac{L_{5} X+L_{6} Y+L_{7} Z+L_{8}}{L_{9} X+L_{10} Y+L_{11} Z+1}
\end{aligned}
$$

$x$ and $y$ are the image pixel coordinates and $X, Y$ and $Z$ are the $3 \mathrm{D}$ coordinates of the reference points. The coefficients $L_{1}$ to $L_{11}$ are the DLT parameters to be estimated. From these parameters, three interior orientations and six exterior orientations parameters can be derived. The two remaining elements describe the shearing and scaling of the affine transformation. The equation to derive the orientation parameters from the DLT parameters the book of Luhmann et al. (2006) 
is referred. By rearranging Equation 3.20, the following linear system is obtained:

$$
\begin{gathered}
L_{1} X+L_{2} Y+L_{3} Z+L_{4}-x L_{9} X-x L_{10} Y-x L_{11} Z-x=0 \\
L_{5} X+L_{6} Y+L_{7} Z+L_{8}-y L_{9} X-y L_{10} Y-y L_{11} Z-y=0
\end{gathered}
$$

In order to solve this system of equations for $n$ reference points $(n \geq 6)$, a LSQ model of $y=A \hat{x}+e$ can be used, were the $y$ is the observation vector, $A$ the design matrix and $e$ the residuals. The design matrix $A$ is composed as follows:

$$
\left[\begin{array}{ccccccccccc}
X_{1} & Y_{1} & Z_{1} & 1 & 0 & 0 & 0 & 0 & -x_{1} X_{1} & -x_{1} Y_{1} & -x_{1} Z_{1} \\
0 & 0 & 0 & 0 & X_{1} & Y_{1} & Z_{1} & 1 & -y_{1} X_{1} & -y_{1} Y_{1} & -y_{1} Z_{1} \\
X_{2} & Y_{2} & Z_{2} & 1 & 0 & 0 & 0 & 0 & -x_{2} X_{2} & -x_{2} Y_{2} & -x_{2} Z_{2} \\
0 & 0 & 0 & 0 & X_{2} & Y_{2} & Z_{2} & 1 & -y_{2} X_{2} & -y_{2} Y_{2} & -y_{2} Z_{2} \\
\vdots & \vdots & \vdots & \vdots & \vdots & \vdots & \vdots & \vdots & \vdots & \vdots & \vdots \\
X_{n} & Y_{n} & Z_{n} & 1 & 0 & 0 & 0 & 0 & -x_{n} X_{n} & -x_{n} Y_{n} & -x_{n} Z_{n} \\
0 & 0 & 0 & 0 & X_{n} & Y_{n} & Z_{n} & 1 & -y_{n} X_{n} & -y_{n} Y_{n} & -y_{n} Z_{n}
\end{array}\right]
$$

In order to solve the eleven DLT parameters, a minimum of six reference points are required. Since the Equations 3.22 are linear, no approximate values are required to solve the unknown parameters. Because of the affine transformation applied to the measured image coordinates, there is no need for a fixed coordinate system defined in the camera. Instead, it is directly possible to use measurements made by an arbitrary measuring device with non orthogonal axes and different axial scale factors e.g. pixel coordinates. Images made from non metric cameras, which have no image coordinate system or have an unknown interior geometry, can also be evaluated by this method.

\subsubsection{D Object Reconstruction}

Once the DLT parameters are calculated the object coordinates can be calculated using the standard DLT Equations 3.20. In this case the DLT parameters and the image coordinates are known and the object coordinates $(X, Y, Z)$ need to be estimated. Since their are three unknowns to be solve and per image only two observation are available, the image coordinates $(x, y)$ of the object point, a second image of the 
object is needed to gain four observation resulting in a redundancy of one. The model $y=A \hat{x}+e$ to solve the object coordinates is composed as follows:

$$
\left[\begin{array}{c}
x_{1}-L_{4} \\
y_{1}-L_{8} \\
x_{2}-L_{4} \\
y_{2}-L_{8}
\end{array}\right]=\left[\begin{array}{ccc}
L_{1}-x_{1} L_{9} & L_{2}-x_{1} L_{10} & L_{3}-x_{1} L_{11} \\
L_{5}-y_{1} L_{9} & L_{6}-y_{1} L_{10} & L_{7}-y_{1} L_{11} \\
L_{1}-x_{2} L_{9} & L_{2}-x_{2} L_{10} & L_{3}-x_{2} L_{11} \\
L_{5}-y_{2} L_{9} & L_{6}-y_{2} L_{10} & L_{7}-y_{2} L_{11}
\end{array}\right]
$$

The accuracy of the unknowns $(\hat{x})$ in the LSQ can be calculated using linear error propagation of the errors in the observation vector $\left(\sigma y^{2}\right)$. The equation to calculate the accuracy of $\hat{x}$ is (Teunissen, 2003):

$$
\sigma_{\hat{x}}^{2}=\left(A^{*} Q_{y} A\right)^{-1}
$$

were $Q_{y}$ is the covariance matrix containing the variance (accuracy) of the observations in the diagonal elements.

Unfortunately the standard error propagation rules of LSQ cannot be used to calculate the errors of the object coordinates calculated with DLT. These equations are only valid if all errors of the observations are only present in the observation vector $y$. The design matrix $A$ in Equation 3.22 used estimate the DLT parameter also contained observations, which have an error. For these observations in the design matrix no errors are added in the error matrix $Q_{y}$ and therefore this error propagation method cannot be used.

An alternative method to get an estimate of the accuracy of the calculated object coordinates is to calculate the residuals in the LSQ. The residuals can be calculated as follow (Teunissen, 2003):

$$
\begin{aligned}
& \hat{e}=y-\hat{y} \\
& \hat{e}=y-A \hat{x}
\end{aligned}
$$

The residuals give an estimate of the quality of the fitted model. By calculating the mean and standard deviation of the residuals an overall accuracy of the calculated object coordinates can be obtained.

\subsection{Vibration Measurement of Reconstructed 3D Position}

Using the photogrammetric techniques described in the previous section 3D positions, of the targets pleased on the blades of the wind turbine, 
can be reconstructed from images made from two cameras. At normal operational conditions, the high speed acquisition frequency allows to measure the blades position dense enough, such that all motions of the wind turbine can be captured. By tracking the 3D position of a target in all the frames the vibration of the target can be derived. There are three main types of vibrations:

- flapwize vibrations are vibrations of the blade perpendicular to the rotational plane of the blade;

- edgewise vibrations are vibrations of the blade in the rotational plane;

- structural vibrations are vibrations of the whole structure of the wind turbine.

Deriving all these vibrations from the tracked positions is out of the scope of this thesis. To analyze the measuring capabilities of photogrammetry the most important vibration type, the flapwise vibration, will be calculated.

If the blades of the wind turbine would rotate without any vibrations, the track of a target would lie in a plane. But due to the vibrations the track of the target deviates from this ideal plane. Since the blade is an elastic object, the vibrations will have a goniometrical function. So if a LSQ plane is fitted to the 3D position of the tracked target, this should coincide with the plane without any vibrations. The vibrations of the plane can be measured by the distance between the object coordinates of the targets and the LSQ fitted plane. A mathematical method to fit a LSQ plane to a dataset and measure the distance of the points of the dataset to the LS plane is PCA.

PCA is mathematically defined as an orthogonal linear transformation that transforms a given set of coordinates to a new coordinate system, such that the greatest variance by any projection of the data comes to lie on the first coordinate (called the first principal component), the second greatest variance on the second coordinate, and so on. PCA is theoretically the optimum transformation for a given data set in least square terms. A more detailed description of PCA can be found in Jolliffe (2002).

Using PCA, the object coordinates of the tracked target are transformed into a new coordinate system, such that the new $X Y$ plane is defined by the rotational plane and the flapwise vibrations are defined 
along the $Z$ axis. In Figure 3.18 an example is given of the three principle components (the magenta arrows) of the positions (the blue dots) of a tracked target. The green plane is the LSQ fitted plane, consisting of the first and second principal components and the black lines are the vibrations in the direction of the third principal component.

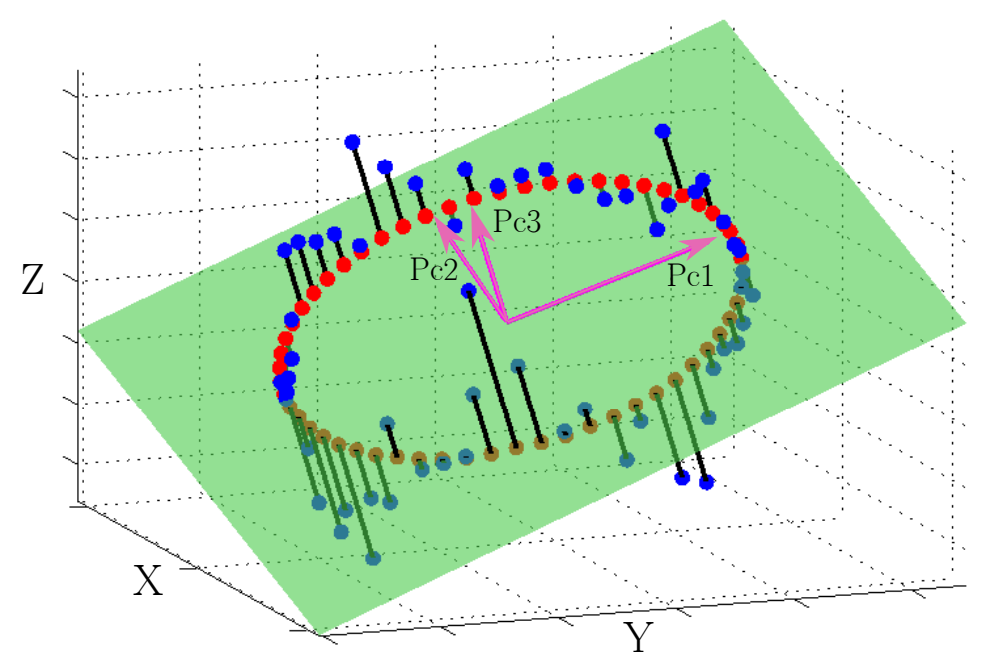

Figure 3.18: The principal components of the tracked positions of a target to derive flapwise vibrations

To determine the accuracy by which the vibrations can be measured, it is necessary to transform the errors from the $3 \mathrm{D}$ coordinates to the principal components. The error estimation of the 3D coordinates will be discussed in Section 3.3.6. Since the transformation to the principal components is linear, the errors in the principal components can be computed using linear error propagation (Teunissen, 2003):

$$
\sigma_{\hat{x}}^{2}=\left(A^{*} Q_{y}^{-1} A\right)^{-1}
$$

were $A$ is the transformation matrix of the $\mathrm{PCA}, Q_{y}$ is the error matrix containing the errors of the $X, Y$ and $Z$ coordinates in the diagonal matrix and $\sigma_{\hat{x}}^{2}$ is the calculated error in the three principal components. 


\section{Chapter 4}

\section{Automated Target Measurement and Tracking}

As previously mentioned in the problem statement, the large number of targets measured in images taken at high speed makes manual processing impossible. A method is developed in this thesis to automate the target measurement process. The developed method is based on image processing techniques to detect targets and the physical behavior of the motion to track the targets in a sequence of images. In Section 4.1 a flow diagram of the process of the developed method is given. A detailed description of each processing step is given in the rest of this chapter.

\subsection{Workflow of Automatic Target Track- ing}

As illustrated by Ariyawansa and Clarke (1997) and Kolahi et al. (2007) in Section 1.4, target tracking offers great possibilities to measure a large number of targets in a sequence of images taken at high speed. The tracking concept is based on the assumption that motions are smooth by nature. If the motion is sampled with a high frequency, the position of a target in a sequence of images will change minimally. And if a minimum number of target positions is known from which the motion can be modeled mathematically, the position in the next image can be predicted. A wind turbine makes a circular motion and its projection in image space is an ellipse, which can be approximated 


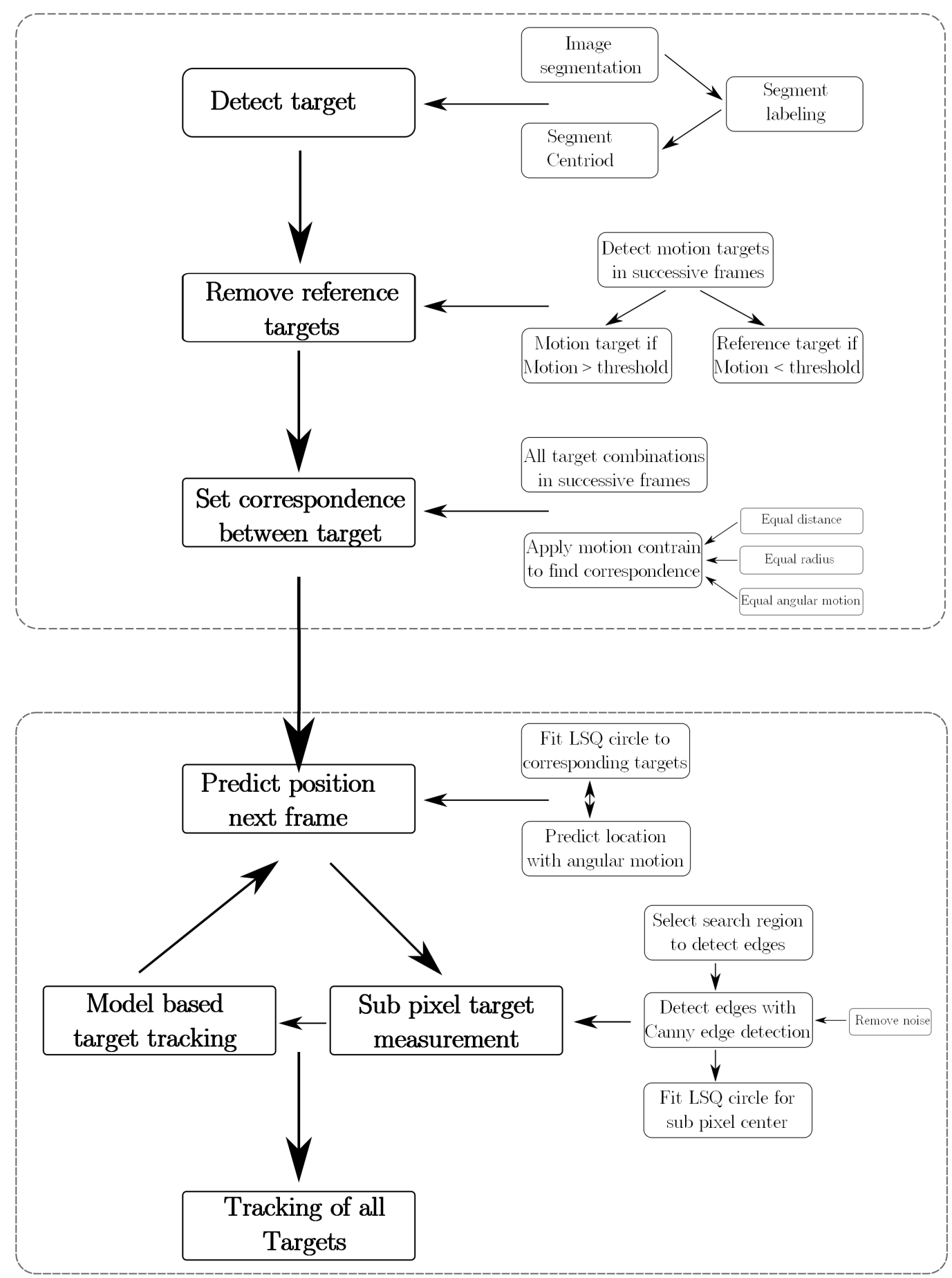

Figure 4.1: Flow diagram for automated target measurement and tracking 
by a circle. For a circular motion at least three target positions are needed to fit the mathematical function in order to track it.

The automatic tracking is performed in two major steps (Figure 4.1):

- detect the targets in the first three images and establish correspondences between them. Also targets that are used as reference, placed on a static object can be rejected, since they do not need to be tracked. The targets are detected by image segmentation. The correspondence between successive targets is established by the constraints of a circular motion.

- The second step is performed by fitting a LSQ circle to the first three positions of a target and predicting the location in the next frame. The center of the predicted target is refined by edge detection and circle fitting.

\subsection{Target Detection}

The first step of the automatic target tracking is to detect the targets in the first three images. From the different methods described in Section 3.2.1 image segmentation is used, as described in Section 3.2.1.
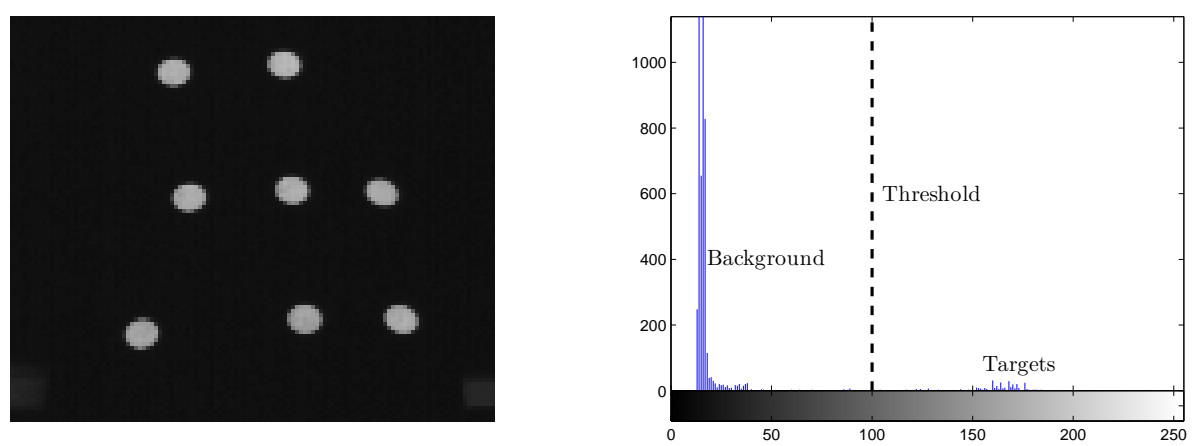

(a) Image of retro reflective targets taken (b) Target detection based on threshold segwith a high speed camera mentation

Figure 4.2: Target detection based on histogram thresholding

Since there are only two main features in the image, a relatively simple target detection technique based on histogram thresholding can be 
used. By constructing a histogram, the two main features can directly be distinguished by the two peaks (Figure 4.2(b)). By using a threshold value, in this case 100, pixels with values smaller the threshold are assigned as background, while pixels with values larger than the threshold are assigned as targets creating a binary image (Figure 4.3(a)).

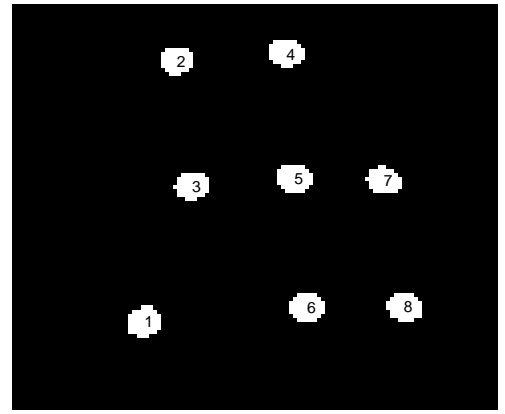

(a) Segmented and labeled binary image

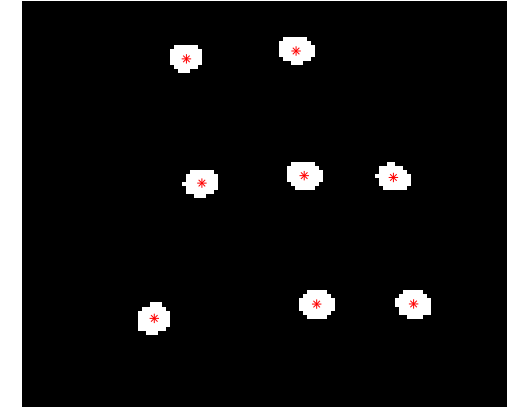

(b) Calculated centroids of the labels or targets

Figure 4.3: The calculated target centers of a binary segmented image

Using a labeling function of MATLAB BwLabel the targets in the binary image are labeled. More details about this function can be found in the help documentation of MATLAB and the book of Haralick and Shapiro (1992).

For the obtained segments or targets, their center is calculated using a centroid method by calculating the average of the number of pixels in the row and column directions (Figure 4.3(b)).

\subsection{Removing Reference Targets}

To monitor the dynamical motions of a wind turbine, retro-reflective targets are put on the blades. For the relative and absolute orientations of images made by the two cameras, additional reference targets were placed on fixed and stable structures.

As the reference targets do not move in the entire sequence of images, it is not important to track them. In the first step of the automatic target tracking, all targets in the first three images are detected, including the 
reference targets. Since the reference targets are not needed, they are removed from the set of detected targets.

The reference targets are removed as follows:

- by calculating the difference in position for every target in the first image with all targets in the second and third image;

- if for a target in the first image the difference in position with a target in the second or third image is smaller then a threshold, that target is considered to be a reference target and is therefore removed.

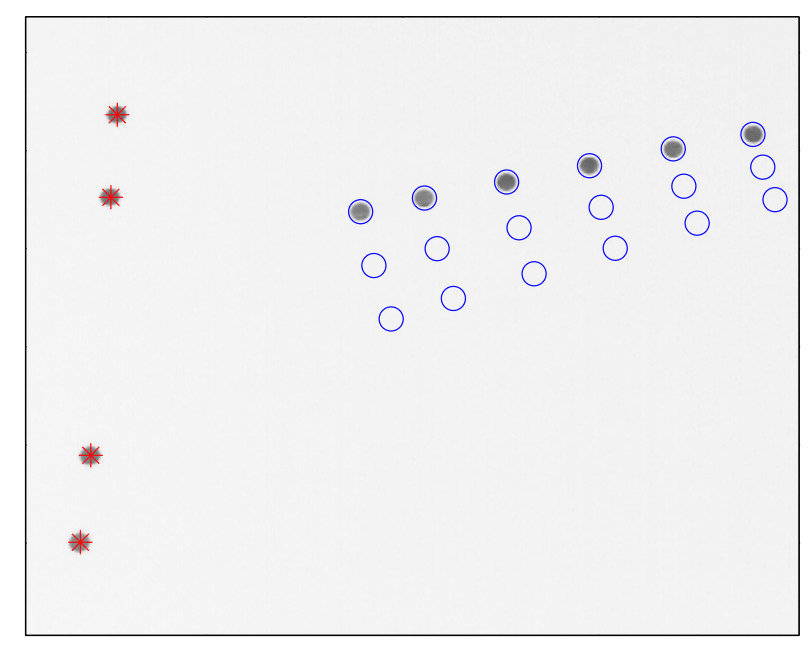

Figure 4.4: Reference and blade targets distinguished by a threshold of movement

In Figure 4.4, an example is given where the reference targets are distinguished from moving targets. The red stars are the reference targets and have the same position in the three frames, while the blue circles are the moving targets and their position changes in every frame. In this example, a moving threshold of one pixel is used to distinguish both types of targets. 


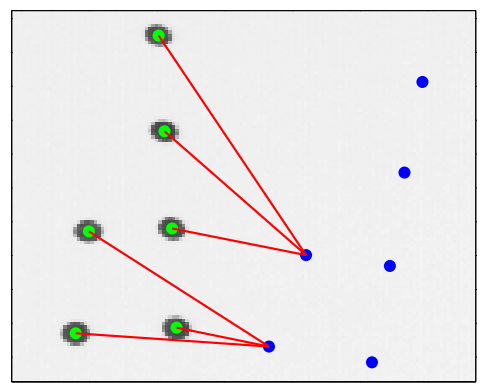

(a) Invalid correspondences created for targets where the motion between frames is larger than the targets spacing

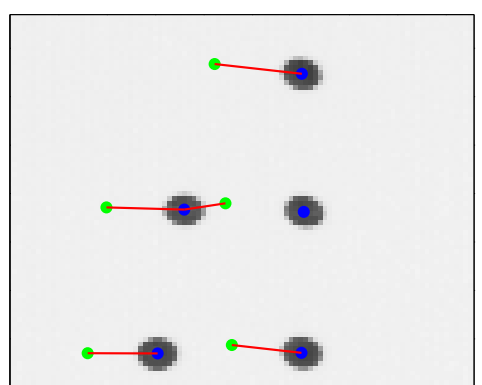

(b) An invalid correspondence created for a target where the position of a neighboring target is the nearest neighbor

Figure 4.5: Sensitivity of using nearest neighbor to establish correspondence

\subsection{Establishing Correspondences Between Targets of Successive Frames}

After removing the reference targets, the next step is to establish correspondences between the moving targets. A simple method to establish correspondence is to find for each target in image one the nearest target in image two. The limitation of this approach is that it cannot be used if the motion between the targets is larger then the distance between the targets. In Figure 4.5(a) an example is given of this situation. The green dots are the positions in the first frame, the red dots are the positions in the second frame and the red lines show the established correspondences. In Figure 4.5(b) another example is given, where the motion between the targets is smaller then the distance. Even in this case there is one target for which the nearest neighbor is incorrect.

Since this automated process is intended to detect motions of a wind turbine, correspondences can be found by applying physical constraints of the known motion. The blades of a wind turbine make a circular motion in object space, but in image space this can deform to an ellipse. A small segment of the ellipse between a sequence of three target positions can also be locally approximated with a circle.

To find the right correspondences between the targets of the three images, the first image is used as a reference. For every target in the first image, all possible combinations are made with targets from the 
second and third image. To all these possible combinations, a set of constraints are applied to eliminate incorrect correspondence combinations. The constraints are based on the assumption that the wind turbine rotates with a constant speed and the images are taken with a constant time interval. The constraints applied to the combination of successive targets are as follows:

\section{(a) equal radius}

the distance of the targets to the center of motion should be equal. In Figure 4.6(a), the green lines illustrate a combination with equal radius. By using an equal radius constraint, incorrect combinations in the radial direction of the turbine are removed.

The threshold used here cannot be set very strict (close to zero), because the circular motion of the targets further away from the image center have a larger elliptical distortion than the targets close to the image center. By using a larger threshold, combinations of targets that are close to each other will be considered as correct and will be not eliminated. The red line in Figure 4.6(a) illustrate such an incorrect combination;

(b) equal angular motion

the angular motion with respect to the center of motion should be equal. In Figure 4.6(b), the green lines illustrate a combination with equal angular motion. This constraint removes incorrect target combinations that are close to each other and parallel to the motion. Also here a threshold is used.

Since the motion is elliptical and not circular, the threshold value cannot be set very strictly. This causes that incorrect combinations of targets close to each other will have an angular motion approximately equal to correct one and will therefore not be eliminated. The red lines in Figure 4.6(b) illustrate such an incorrect combination;

\section{(c) angular motion $\approx$ mean angular motion}

the angular motion of a target with respect to the center of motion should be equal to the mean angular motion of all targets. In Figure 4.6(c), the green lines illustrate a combination with an angular motion equal to the mean angular motion of all combinations.

The two previous constraints will have eliminated most of the incorrect combinations, therefore it can be assumed that the mean of all combinations will be close to the actual angular motion, which is equal for all targets. The mean angular motion can also be 
further refined by an outlier filtering technique such as RANSAC (described in Section 4.6.2). By using this constraint, the incorrect target combinations that are close to each other and parallel to the circular motion can be eliminated;

(d) closest target

using the previous constraints, almost all incorrect combinations of target positions are removed. Theoretically it is still possible that an incorrect combination is not detected and therefore two combinations will remain for a target. To choose the most likely combination from these two, the closest target to path constraint can be used.

This constraint is based on the fact that a small segment of a circle can be locally approximated by a straight line. For the remaining combinations, a line (path) is constructed to the positions in the first and second frames. The closest target position to the path in the third frame belongs to the right combination. In Figure 4.6(d) an example is given where the green line is the closest to the dotted path going through the first and second target positions.

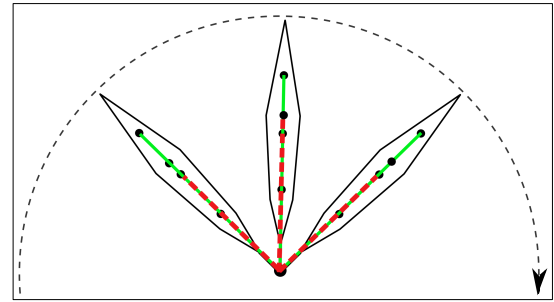

(a) Equal radius

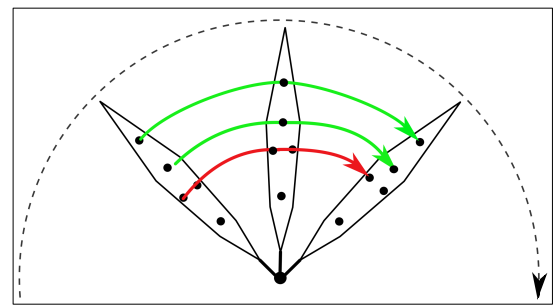

(c) Angular motion $\approx$ mean angular motion

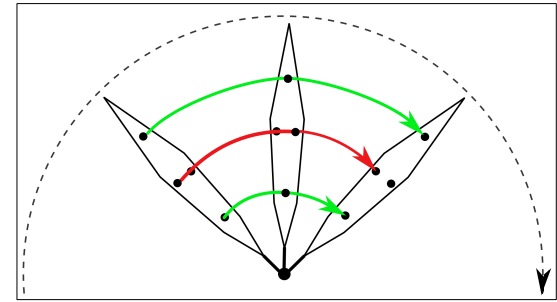

(b) Equal angular motion

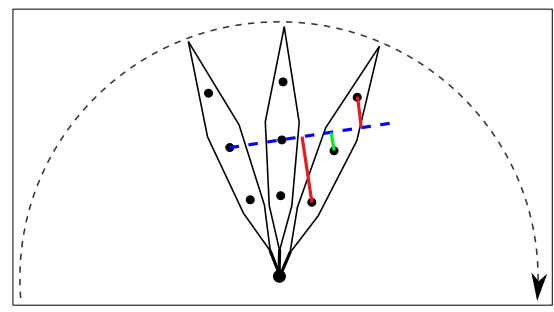

(d) Targets close

Figure 4.6: Constraints to find corresponding targets in three successive images 


\subsection{Model Based Target Tracking}

Once the correspondences between the targets of the first three images are established, a least squares circle can be fitted and the positions of the targets in the next frame can be predicted. In Figure 4.7, the circular targets (in magenta) illustrate the known positions of a target in the previous three frames and the blue large circle the least squares fitted circle. The position in the current frame is illustrated by the green square, and is predicted by the angular motion calculated from the target positions in the previous frames and the center of the fitted circle.

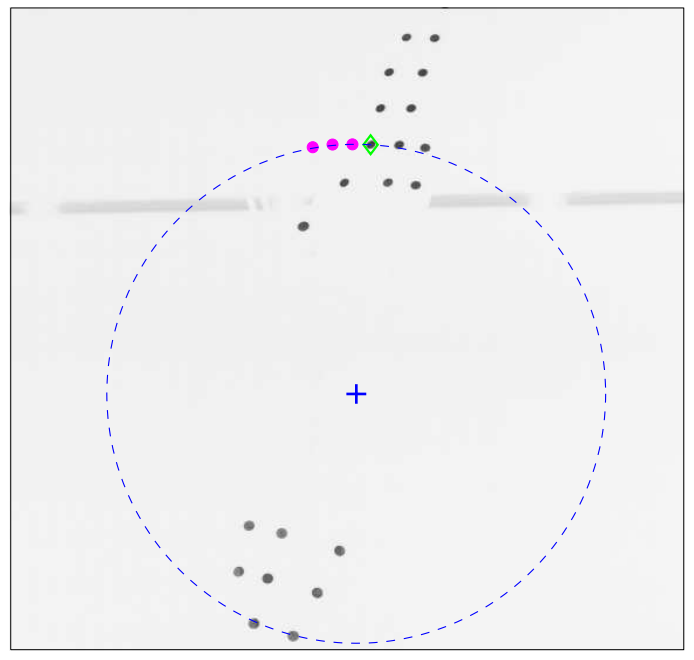

Figure 4.7: Predicted target position by a LSQ fitted circle to the three previous known positions and the angular motion between them

\subsection{Target Measurement With SubPixel Precision}

Once the targets in the fourth image are predicted the tracking begins. Based on the new position and the already known positions of the previous frames, the position in the next frame can be estimated.

In Figure 4.8(a), a close up view is given of a predicted target. It can be noticed that the predicted position illustrated by the red star is not 


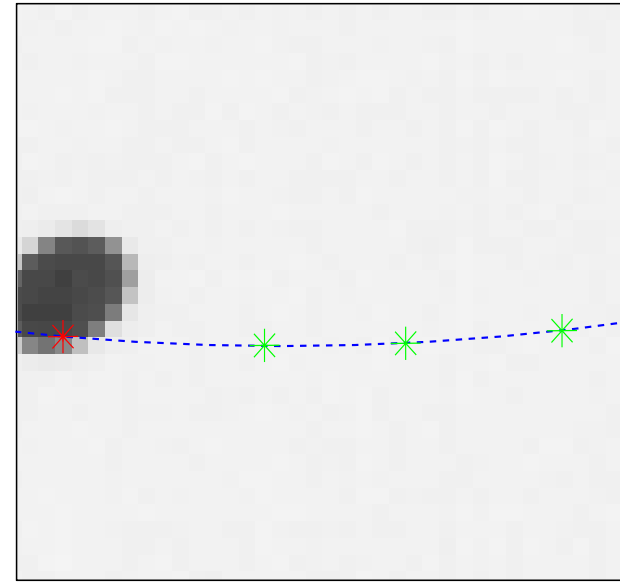

(a) Predicted target location

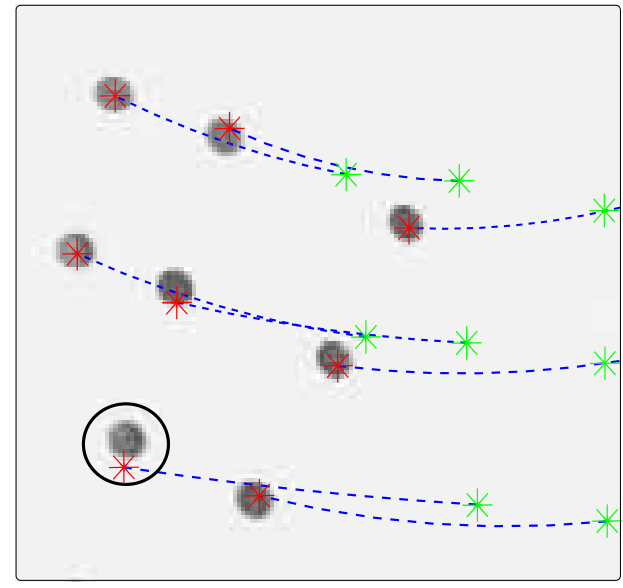

(b) Unsuccessful target tracking due to error accumulation

Figure 4.8: Unsuccessful tracking caused by imprecise targets measurement

at the center of the target. If this position is used to predict the target position in the next frame, this error will accumulate. If the center of the target is not corrected after a few iterations, the prediction will lie outside the target for example the circle target in Figure 4.8(b).

In order to track the targets successfully in a sequence of images, it is important to correct the center of the targets to subpixel precision. This subpixel precision is also necessary to calculate precise 3D coordinates in object space of a target, which is directly related to the accuracy by which the vibrations of the blades of a wind turbine can be measured.

The subpixel target measurement is performed in four steps:

(a) at first, a window is selected around the predicted location to detect the edges;

(b) within the selected window, the edges are detected;

(c) there might be irrelevant edge pixels not belonging to the target in the selected window, these need to be filtered out;

(d) a circle is fitted to the filtered edges to calculate the center of the target with subpixel precision. 


\subsubsection{Edge Detection}

From the predicted location, a rectangular region containing the target is selected by a template window. The size of the template window should be bigger than the target, but small enough that it doesn't have any overlap with other targets. In Figure 4.9(a), an example is given of the template window size. The blue template window is big enough to contain the whole target, and small enough that it doesn't overlap with other targets, while the red template window is so big that it covers other targets that do not belong to the predicted target.

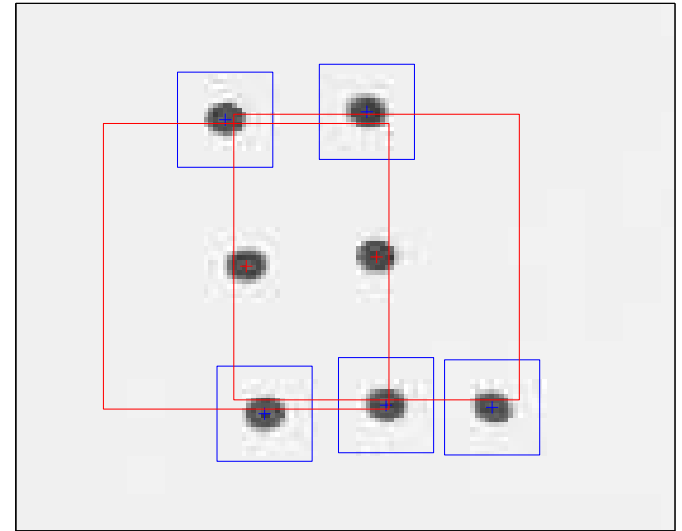

(a) Size of search window to find edges

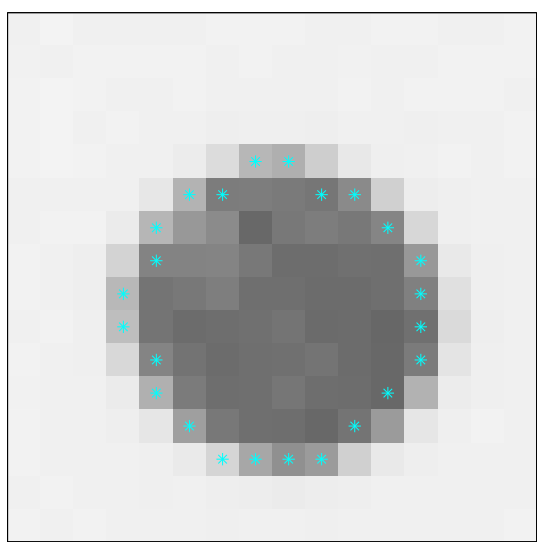

(b) Detected edges with the Canny edge detector

Figure 4.9: Window size used to detect edges with Canny edge detector

After the search window is selected, the edges are detected using the Canny edge detection method (Figure 4.9(b)). This method is already described in Section 3.2.1.

\subsubsection{Pin-Pointing Target Center}

If the shape of a target in object space is a circle in image space, this can also be approximated by a circle. In order to pin-point the center of the target with subpixel precision, a LSQ estimated circle can be fitted to the detected edges (Figure 4.10). The circle is fitted with the same procedure as described in Section 3.2.1. The red star in Figure 4.10 illustrate the center of the fitted circle. 


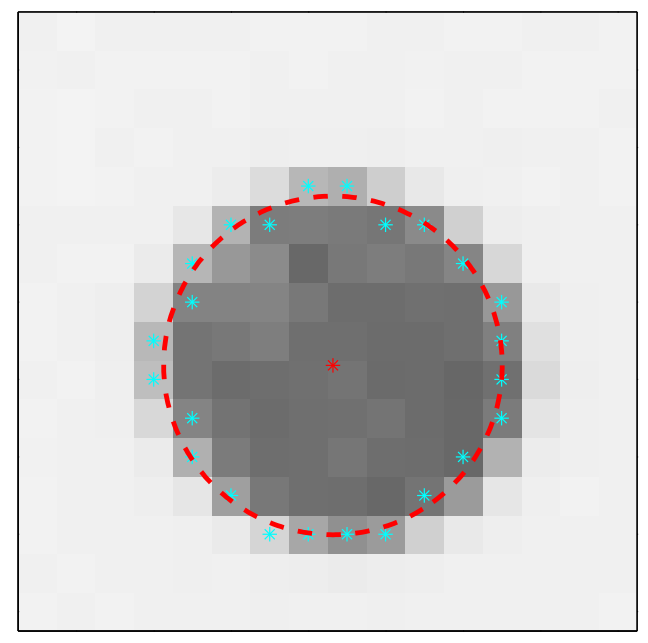

Figure 4.10: LSQ circle fitted to detected edges of a target

\subsubsection{Eliminating Outlying Edge Pixels using RANSAC}

As previously discussed in Section 3.2.1, only reflective targets illuminated by artificial light will be visible in images taken at high speed. However, in reality there might be other materials in the viewing range of the camera, which are able to reflect light with enough intensity to be captured by the sensor. The dark gray bar in Figure 4.11 is an example of an undesired feature in the image caused by a light bulb. Neighboring targets can also cause outlying edges if the size of the window selected around the target is too large.

If in the tracking process a target location is predicted close to this undesired feature, its edges will also be detected (Figure 4.12(a)). If a circle is fitted to these edges, it will result in a wrong target center. In order to solve this problem, these undesired outlying edges can be removed using RANSAC.

The outlying edges from a neighboring target can be prevented by making the size of the selected window smaller, such that it only contains the wanted target. In case the template window contains only a part (below 50\%) of the neighboring target, this can also be detected and removed using RANSAC.

Random Sample Consensus (RANSAC) is an algorithm to robustly fit 


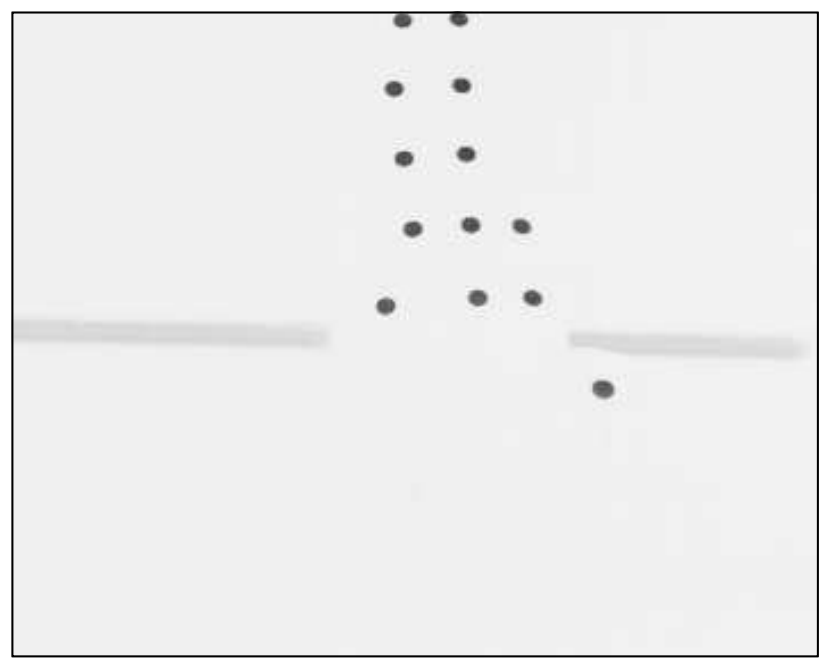

Figure 4.11: Undesired features in a high speed image

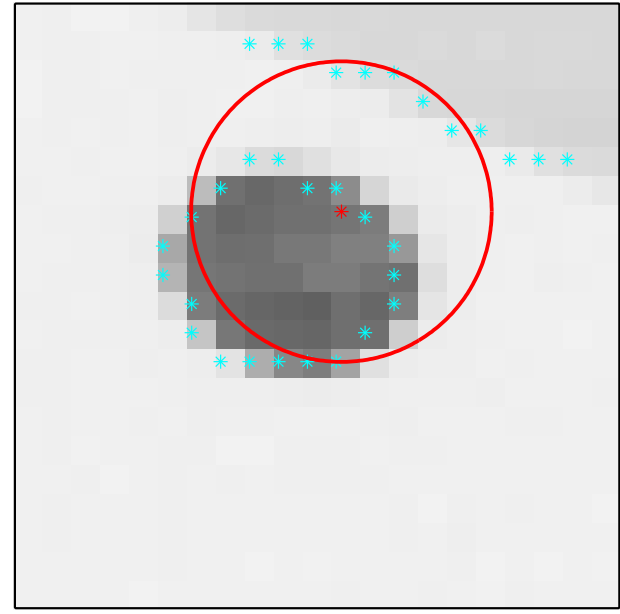

(a) Edges detected from an undesired image feature

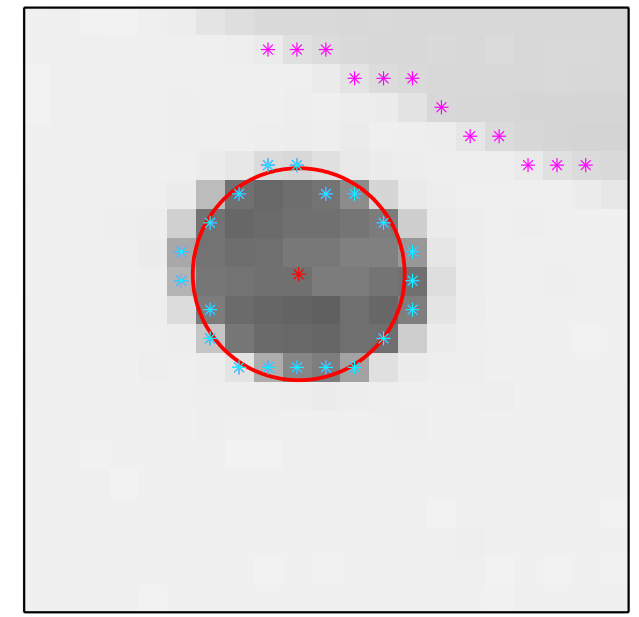

(b) Outlying edge pixels removed with RANSAC

Figure 4.12: Fitting a circle in the presence of noise 
models to experimental data (Fischler and Bolles, 1981). To remove the outliers and pin-point the target center, a circle is used as model. This algorithm using a circle as model works as follows:

(a) three edge points, the minimum to fit a circle, are selected randomly;

(b) then the support for this fitted circle is measured by the number of points that lie within a distance threshold $(t)$ of the circle;

(c) this random selection is repeated a certain number of times $(N)$;

(d) the circle with the most support is considered to be the most robust fit. The idea is that if one of the randomly selected points is an outlier the selection will not gain much support;

(e) the outliers of this selection are considered to be noise and are removed from the original set of edges;

(f) for the left over edge points, a final circle is fitted ( Figure 4.12(b)).

The threshold distance $t$ can be calculated statistically with a probability of $\alpha$. This calculation requires a probability distribution function for the distance from the model to the inliers. However, in practice this distance is chosen empirically.

The number of samples $N$ is chosen to ensure with a probability $p$ that at least one of the random samples is free from outliers. In Table 4.1, an overview is given of the sample size with respect to the ratio of noise to get a probability of $p=0.99$.

Table 4.1: At a sample size of three the number of samples required at a certain proportion of outliers

\begin{tabular}{lccccccc}
\hline \hline Proportion of outliers & $5 \%$ & $10 \%$ & $20 \%$ & $25 \%$ & $30 \%$ & $40 \%$ & $50 \%$ \\
\hline Number of samples & 3 & 4 & 7 & 9 & 11 & 19 & 35 \\
\hline
\end{tabular}

\subsubsection{Circle and Ellipse Fitting}

In Section 4.6.2, an assumption was made that a projection of a circular target in an image can be approximated by a circle. In reality, these circles are deformed to ellipses due to the perspective projection and lens distortion. In Figure 4.13(a), an example is given of a target close to the image center which therefore has few deformations. The shape 
of this target can sufficiently be approximated by a circle, while the target in Figure 4.13(b) has been deformed and the shape can only be approximated with an ellipse.

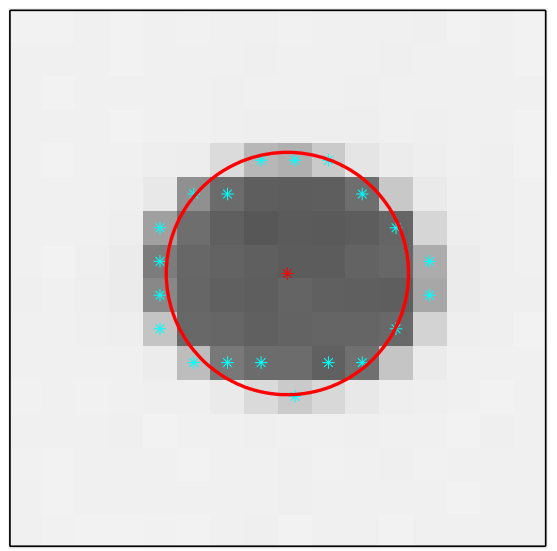

(a) Proximately Circular target

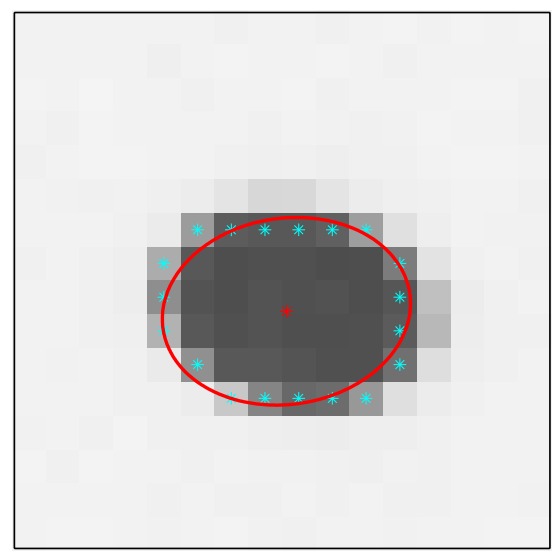

(b) Target deformed to an ellipse

Figure 4.13: Deformed shapes of a circular target

Knowing the deformations of a circular target in image space, it can be concluded that fitting an ellipse is better then fitting a circle to model the shape of the target. However, the goal in this study is not to model the shape, but to pin-point the center of the target with subpixel precision. To determine if there is a difference between the target centers calculated by a circle or an ellipse a test is performed.

In the test a LSQ circle and ellipse are fitted to a set of points generated along an ellipse. By measuring the deviation between the centers pinpointed by the circle and ellipse, it can be determined if a circle is sufficient to pin-point the target center. The set of points along an elliptical target are generated as follows:

(a) The minimum number of 5 edge points to fit an ellipse are randomly are generated;

(b) 360 evenly distributed edge points are generated;

(c) 20 random edge points are generated;

(d) 20 evenly distributed edge points are generated.

The results and analysis of this test will be given in Section 6.1.6. 


\section{Circle Fitting}

There are several methods to define a mathematical function of a circle e.g. with polar coordinates, trigonometric and Cartesian coordinates. Cartesian coordinates are commonly used, where the function is defined by the midpoint $(a, b)$ and the radius $r$ of the circle (Equation 4.1). These are also the parameters, which need to be estimated in the LSQ model.

$$
\left(x^{2}-a\right)+\left(y^{2}-b\right)=r^{2}
$$

Since the function of a circle is nonlinear, it is necessary to linearize the equation for the Least Squares Estimation (LSQ). In literature there are many methods described to linearize and fit a circle to a set of data points. In this study a method is used where the set of data points $\left\{\left(x_{i}, y_{i}\right) \mid 0 \leq i<N\right\}$ are normalized to $(u, v)$ by subtracting it from the mean $(\bar{x}, \bar{y})$ (Bullock, 2006). The unknown parameters are first estimated in $(u, v)$ coordinates and then transformed back to original $(x, y)$ coordinates.

$$
\begin{aligned}
u_{i} & =x_{i}-\bar{x} \\
v_{i} & =y_{i}-\bar{y} \quad \text { for } 0 \leq i<N
\end{aligned}
$$

If the center of the circle is defined by $\left(u_{c}, v_{c}\right)$ and radius $R$, then the function which needs to be minimized is $S=\sum_{i}\left(g\left(u_{i}, v_{i}\right)\right)^{2}$, where $g(u, v)=\left(u-u_{c}\right)^{2}+\left(v-v_{c}\right)^{2}-\alpha$ and $\alpha=R^{2}$. To minimize the function it is differentiated to $S\left(\alpha, u_{c}, v_{c}\right)$.

$$
\begin{aligned}
\frac{\partial S}{\partial \alpha} & =2 \sum_{i} g\left(u_{i}, v_{i}\right) \frac{\partial g}{\partial \alpha}\left(u_{i}, v_{i}\right) \\
& =-2 \sum_{i} g\left(u_{i}, v_{i}\right)
\end{aligned}
$$

Thus $\partial S / \partial \alpha=0$ if

$$
\begin{gathered}
\sum_{i} g\left(u_{i}, v_{i}\right)=0 \\
\frac{\partial S}{\partial u_{c}}=2 \sum_{i} g\left(u_{i}, v_{i}\right) \frac{\partial g}{\partial u_{c}}\left(u_{i}, v_{i}\right) \\
=2 \sum_{i} g\left(u_{i}, v_{i}\right) 2\left(u_{i}-v_{i}\right)(-1)
\end{gathered}
$$




$$
\begin{aligned}
& =-4 \sum_{i}\left(u_{i}-u_{c}\right) g\left(u_{i}, v_{i}\right) \\
& =-4 \sum_{i} u_{i} g\left(u_{i}, v_{i}\right)+4 u_{c} \sum_{i} g\left(u_{i}, v_{i}\right)
\end{aligned}
$$

In the presence of Equation 4.3, $\partial S / \partial u_{c}=0$ if

$$
\sum_{i} u_{i} g\left(u_{i}, v_{i}\right)=0
$$

Similarly requiring $\partial S / \partial v_{c}=0$ gives

$$
\sum_{i} v_{i} g\left(u_{i}, v_{i}\right)=0
$$

Expanding Equation 4.4 gives

$$
\sum_{i} u_{i}\left[u_{i}^{2}-2 u_{i} u_{c}+u_{c}^{2}+v_{i}^{2}-2 v_{i} v_{c}+v_{c}^{2}-\alpha\right]=0
$$

and by defining $S_{u}=\sum_{i} u_{i}, \sum_{i} u_{i}^{2}$ etc., the equation above can be rewritten as

$$
S_{u u u}-2 u_{c} S_{u u}+u_{c}^{2} S_{u}+S_{u u u}-2 v_{c} S_{u v}+v_{c}^{2} S_{u}-\alpha S_{u}=0 .
$$

Since $S_{u}=0$, this simplifies the equation to

$$
u_{c} S_{u u}+v_{c} S_{u v}=\frac{1}{2}\left(S_{u u u}+S_{u v v}\right)
$$

In a similar fashion, expanding Equation 4.5 and using $S_{u}=0$ gives

$$
u_{c} S_{u v}+v_{c} S_{v v}=\frac{1}{2}\left(S_{v v v}+S_{v u u}\right)
$$

Solving the two linear Equations 4.6 and 4.7 with LSQ the center $\left(u_{c}, v_{c}\right)$ can be obtained. The center of the circle $\left(x_{c}, y_{c}\right)$ in the original coordinate system is calculated using Equation 4.8.

$$
\left(x_{c}, y_{c}\right)=\left(u_{c}, v_{c}\right)+(\bar{x}, \bar{y})
$$

To determine the radius $R$ the Equation 4.3 is expanded

$$
\sum_{i}\left[u_{i}^{2}-2 u_{i} u_{c}+u_{c}^{2}+v_{i}^{2}-2 v_{i} v_{c}+v_{c}^{2}-\alpha\right]=0
$$


Using $S_{u}=S_{v}=0$ again the equation above becomes

$$
N\left(u_{c}^{2}+v_{v}^{2}-\alpha\right)+S_{u u}+S_{v v}=0
$$

From which $\alpha$ can be expressed as

$$
\alpha=u_{c}^{2}+v_{c}^{2}+\frac{S_{u u}+S_{v v}}{N}
$$

$\alpha$ is transformed to the original coordinate system by

$$
R=\sqrt{\alpha}
$$

The calculated midpoint $(a, b)$ of the circle can be used to pin-point the target center in the image, with a subpixel precision.

\subsection{Accuracy Assessment of Method}

In the previous section a method is described to pin-point the target center with subpixel precision. Since the accuracy of the target measurement in image space is directly related to the accuracy of the calculated 3D object coordinates and the vibration derived, it is necessary to determine the accuracy, by which a target can be measured using the method described in Section 4.6 .

Ideally the accuracy can be estimated by comparing the real target center with the pin-pointed target center. Since the real target center is unknown this comparison cannot be done, instead an alternative approach is used.

In this alternative approach an artificial target is made with a known target center having the same spatial and radiometrical dimension as the targets in the images of the wind turbine. By pin-pointing the target center of this artificial target and comparing it with the known target center an estimate can be made of the accuracy by which a target can be measured. The artificial target can be composed as follows:

1. an empty high resolution image is created, such that it is about 100 times larger then the final image containing the target;

2. randomly a target center is chosen of an elliptical target;

3. values are assigned to the pixels of the high resolution image. If a pixel is inside the target a value is assigned to it, which represents the target. If the pixel is outside the target a value is assigned to it, which is representative for the background; 
4. the high resolution image is down sampled using an averaging convolution kernel, such that the dimensions of the target are representative to the target measured of the wind turbine;

5. a point spread function is added to imitate the imperfect focusing of the camera. The kernel of the point spread function can empirically be chosen;

6. motion blur is added to imitate the motion of the targets. The amount of motion blur can be calculated from the shutter speed of the camera and the rotational speed of the blades;

7. Gaussian noise is added to imitate measurement errors of the sensor. The standard deviation of the Gaussian noise can be determined from images taken of the wind turbine;

8. the proposed method in Section 4.6 is used to pin-point a target center of the artificial target;

9. the known target center is translated from the high resolution image to the down sampled image;

10. the difference between the measured and the known target centers indicate the accuracy of the target measurement.

To get a representative estimation of the accuracy of the target measurement, the artificial target can be generated several times with a random target center. 


\section{Chapter 5}

\section{Experimental Results}

In the introduction (Section 1.1) it was already mentioned that this study was initiated as a follow up of an experiment to test the aero-elastic stability limits of a model flexible wind turbine. The aero-elastic stability limits of a wind turbine can be measured by the vibration which occurs on the blades at normal operating conditions. In this experiment the vibrations were measured with a high speed camera. In Section 5.1 the experimental setup is described. The results of the experiment are given in Section 5.2.

\subsection{Experimental Setup}

To perform the aero-elastic stability test, a two blade model wind turbine was used. In Figure 5.1, an illustration is given of the used blade. The dimensions of the blade are $724 \mathrm{~mm}$ for the length, $55 \mathrm{~mm}$ for the minimum chord length and $220 \mathrm{~mm}$ for the maximum chord length. The operational parameters of the blades are:

- a design rotation speed of 420 Rotations Per Minute (rpm) and a maximum rotation speed of $840 \mathrm{rpm}$;

- a design wind speed of 6 meters per second and a maximum wind speed of 20 meters per second;

- a minimum vibration frequency of $20 \mathrm{~Hz}$ and a maximum vibration frequency of $20 \mathrm{~Hz}$.

The test was carried out in a wind tunnel (see Figure 5.2), which blew laminar wind with a constant speed. The position of the wind turbine in the wind tunnel is illustrated in Figure 5.3(a). The wind turbine was put 


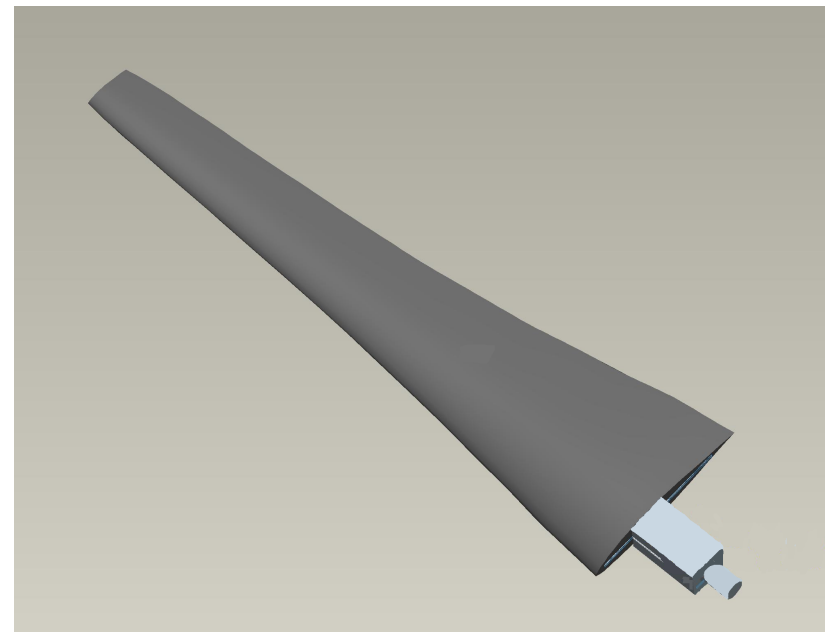

Figure 5.1: The wind turbine blade (Meng, 2009)

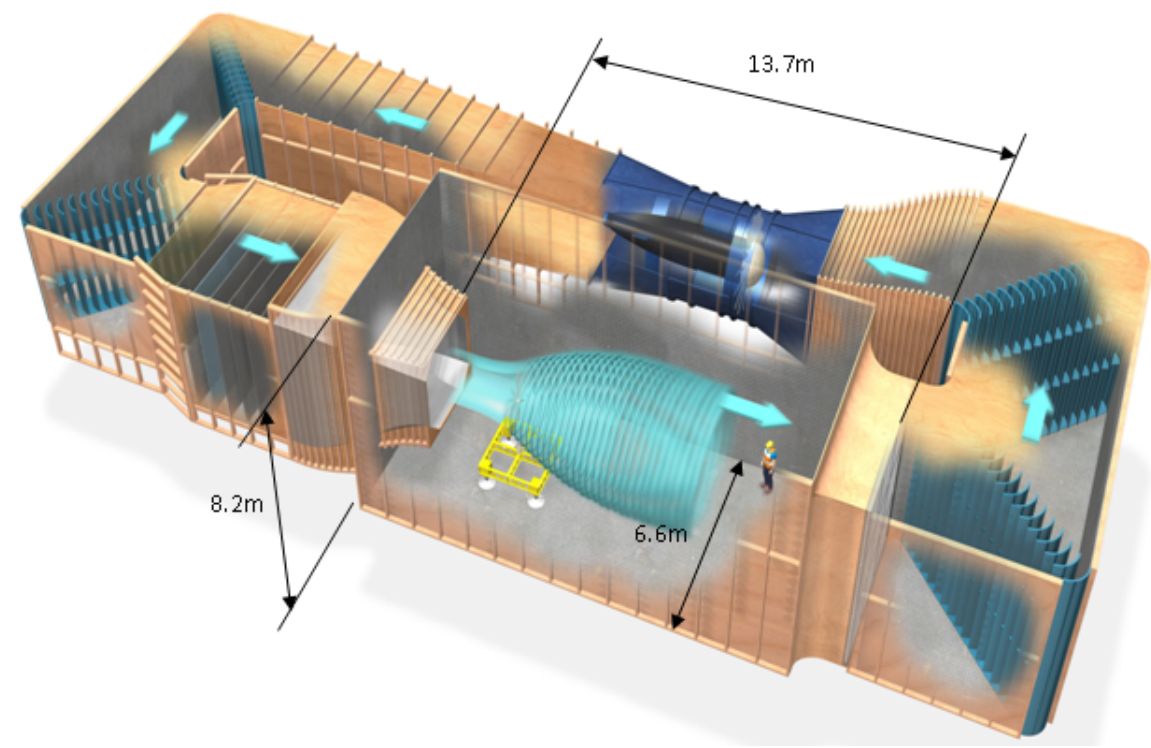

Figure 5.2: Overall layout of the wind tunnel with the dimensions of the experiment chamber 


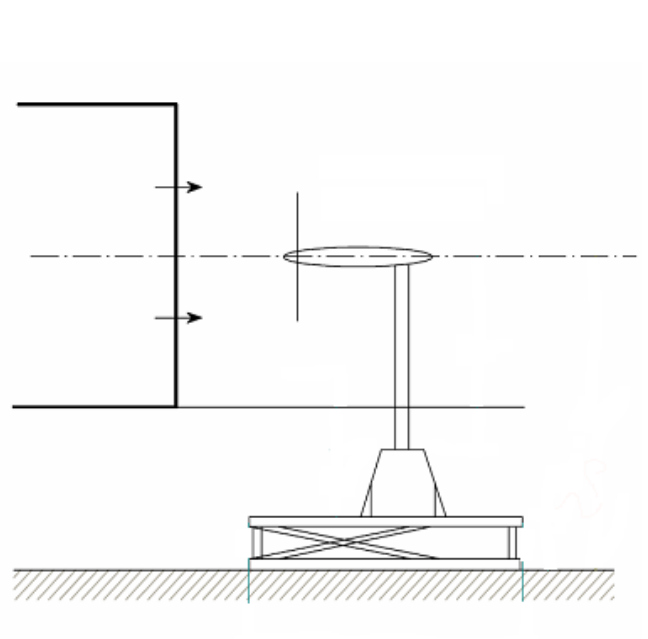

(a) Position of the wind turbine in the wind tunnel (Meng, 2009)

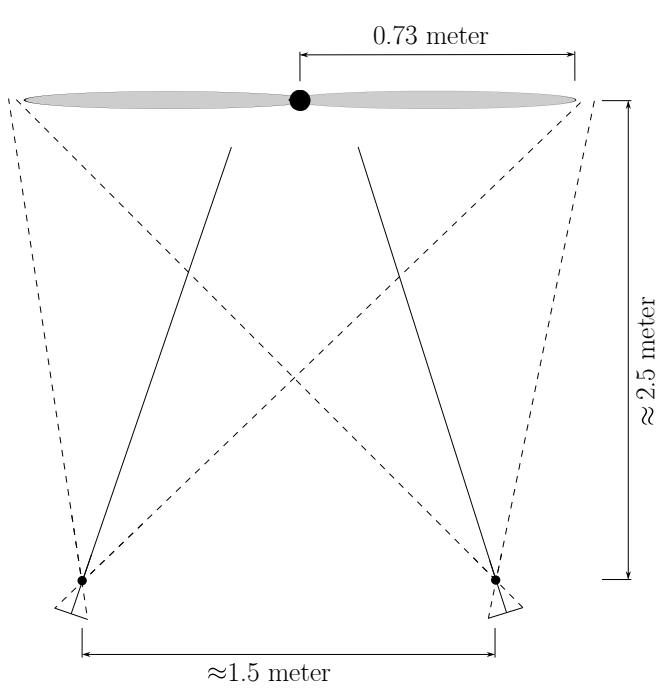

(b) Position of the cameras with respect to the wind turbine

Figure 5.3: A schematic overview of the experimental setup

approximately 2.5 meters in front of the air outlet in order to catch the maximum amount of wind coming out of it.

To measure the vibrations of the wind turbine a pair of high speed cameras were used. The high speed cameras had an acquisition frequency of 500 fps. The lens of the cameras had a focal length of $20 \mathrm{~mm}$ and the imaging sensor had a resolution of 1280 by 1024 pixels. To illuminate the objects in view a powerful flash was used. The distance between the cameras was approximately 1.5 meter.

The position of the high speed cameras is illustrated in Figure 5.3(b). The cameras were put below the air outlet facing the wind turbine. The distance between the camera and the wind turbine was approximately 2.5 meter. The cameras were fixed to the construction of the wind tunnel to eliminate any vibrations to the cameras that could be caused by the blowing wind (see Figure 5.4(a)).

To identify measuring points on the blades of the wind turbine, sixteen retro-reflective targets per blade were put on it. For the interior and exterior orientations, reference targets are needed with a fixed and known position. Thirteen reference targets were placed on a frame constructed around the wind turbine. To calibrate the cameras and determine the scale of the measurement a cross with coded targets with known positions were used (see Figure 5.4(b)). 


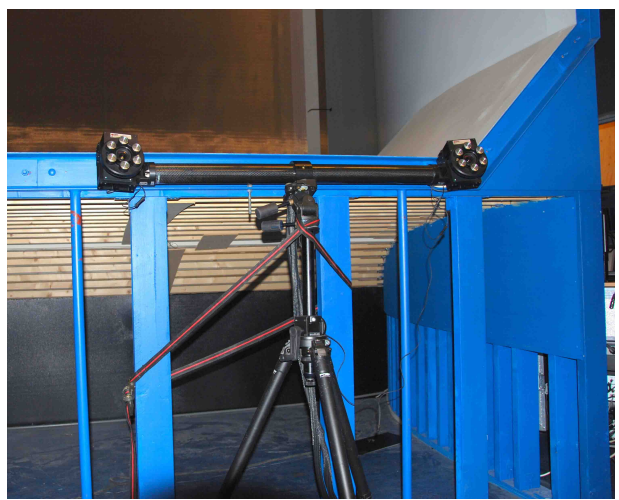

(a) High speed cameras fixed to the construction of the wind tunnel

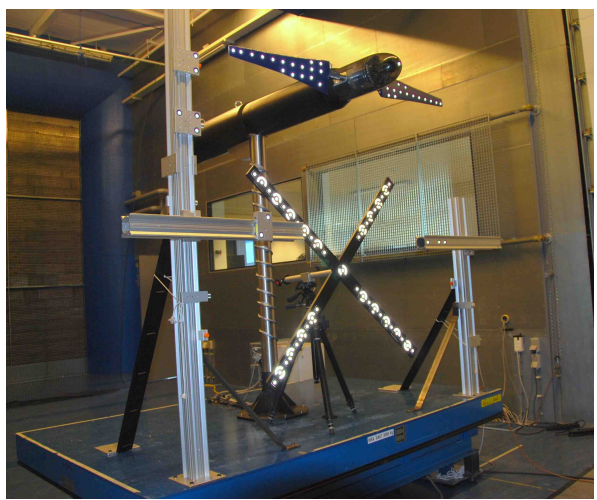

(b) Reference targets put around the wind turbine

Figure 5.4: Experimental setup

The acquisition was done by a contractor company GOM. For this study the company provided one sequence of measured images. The images of the calibration cross, as the coordinates of the reference points were not made available for this study.

The provided sequence of image of the wind turbine were acquired at a rotational speed of $260 \mathrm{rpm}$ and imaging frequency of $500 \mathrm{~Hz}$ for a period of 1.6 seconds. In total 800 frames per camera were captured. In Figure 5.5 the first images made with both cameras are illustrated.

Since no reference targets were made available an alternative approach had to be used to measure reference targets. The software PhotoCore was used to measure coordinates of the targets placed on the blades. Since the two blades of the wind turbine were already unmounted of the rotor, these could not be measured together. One individual blade was used to calculate the object coordinates of the targets in a local reference frame.

The software PhotoCore is able to calculate the object coordinates of circular targets, using twelve coded targets and at least five images taken from different viewing angles. More details about this software the website of PhotoCore (2010) is referred. In Figure 5.6 one of the images is illustrated, which was used to calculate the object coordinates.

\subsection{Results}

In this section an overview is given of the results obtained from the measured images. In Section 5.2.1 the results are given of the targets measured in 


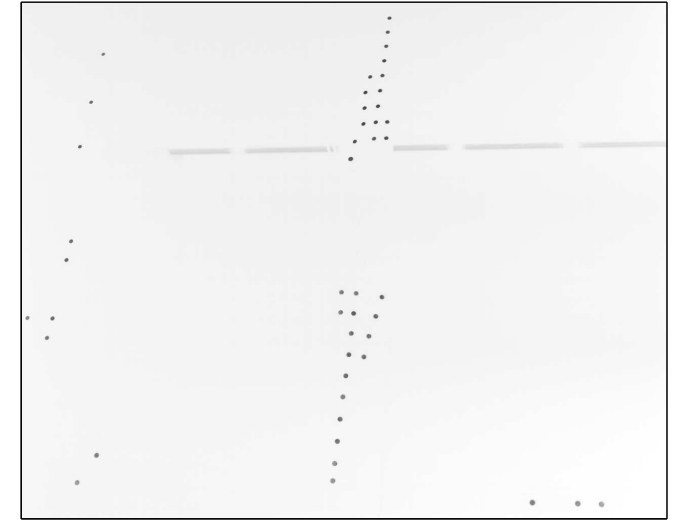

(a) Image made with the left camera

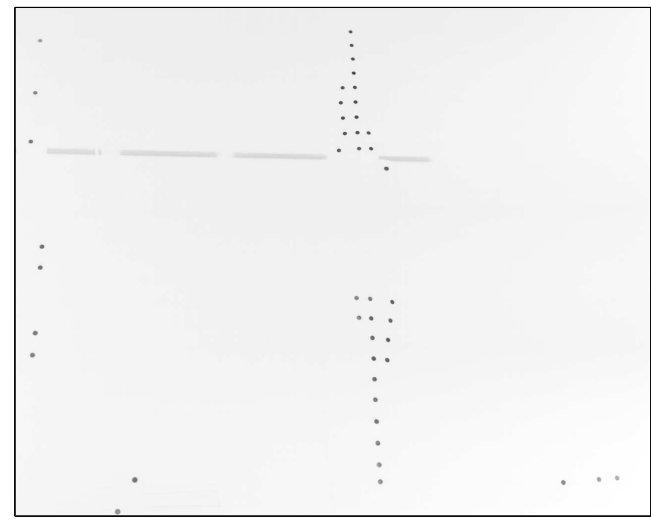

(b) Image made with the right camera

Figure 5.5: Pair of images acquired with two high speed cameras. For better visualization the color scale of the images are inverted from dark to white to white to dark

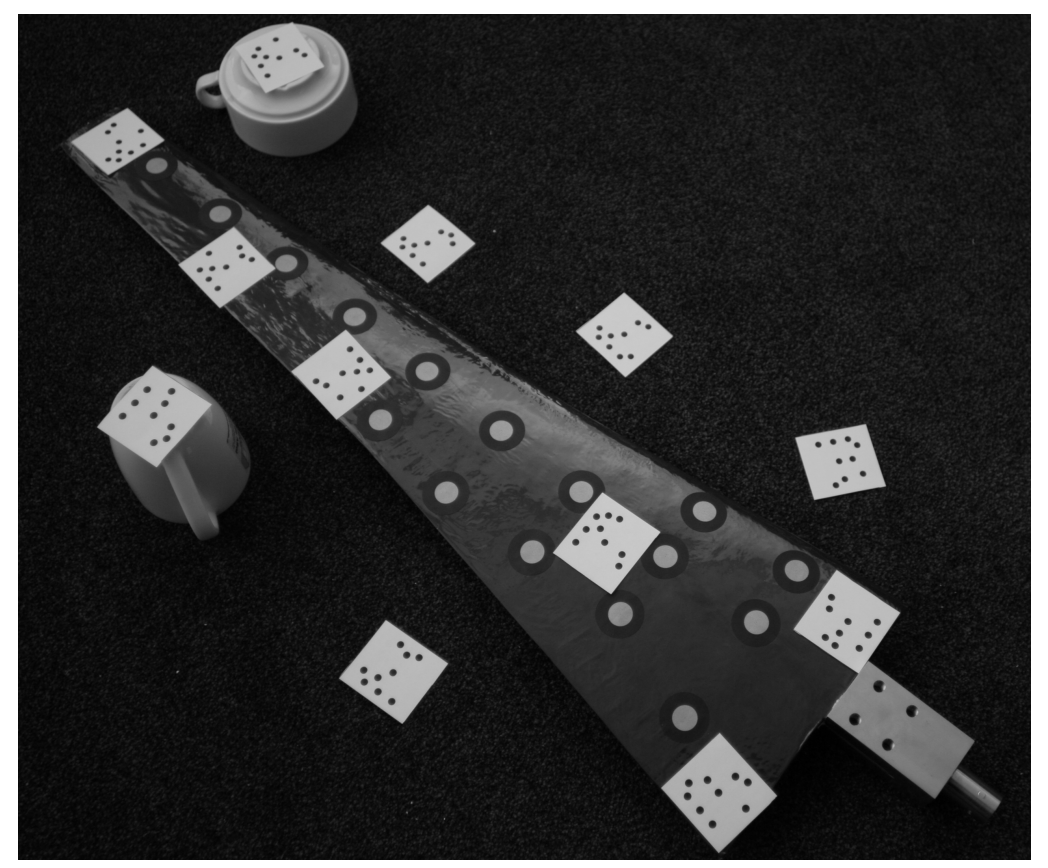

Figure 5.6: Acquisition setup of blade with reference targets to calculate its object coordinates using the software PhotoCore 
image space. The results of the calculated object coordinates are provided in Section 5.2.2. The derived vibrations are described in Section 5.2.3 .

\subsubsection{Results of Automatic Target Measurement and Tracking}

To measure and track the targets automatically the method developed in Chapter 4 is used. The parameters used in the different steps are as follows:

1. for the automatic target detection a histogram threshold value of 50 was used;

2. to remove the reference targets a moving threshold of one pixel was used;

3. to establish the correspondence between the first three frames a threshold of fifteen pixel was used for the equal radius constraint, a threshold of one pixel was used for the constraints equal angular motion and the angular motion $\approx$ mean angular motion;

4. for the target tracking no parameters were needed, since this process is only based on circle fitting;

5. for the edge detection the default parameters of the Canny edge detection of MATLAB was used;

6. to remove outlaying edge pixels RANSAC is used with a distance threshold $(t)$ of two pixels to measure support of a randomly fitted circle. A number of 35 samples were taken, which are needed at a proportion of $50 \%$ of outlaying edges.

In Figure 5.7 the measured position of two targets in the sequence of 115 frames (a complete rotation) are plotted in the first frame. The blue dots illustrate the track of the target with the largest rotational radius, the green dots illustrate the track of the target with the smallest rotational radius and the red dots show the position of the two targets in the first frame. The track of the $x$ and $y$ coordinates of these two targets in the whole sequence of images are respectively given in Figure 5.8(a) and 5.8(b).

From the tracked positions in Figure 5.7 it can be noticed that the circular rotation of the targets in object space is deformed to an ellipse in image space. This distortion is caused by the perspective projection and possible lens distortions. 


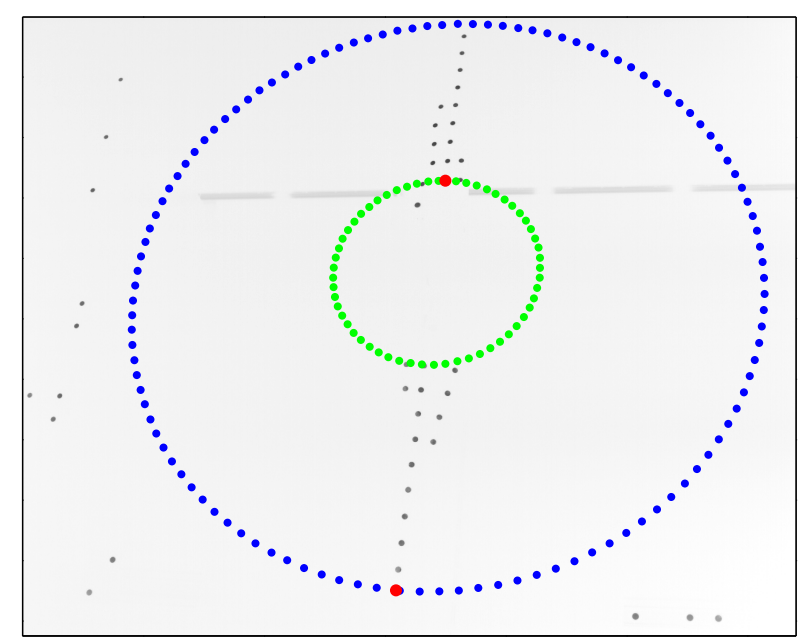

Figure 5.7: Tracked and measured position of two targets in the sequence of 115 images are plotted in the first image of the sequence

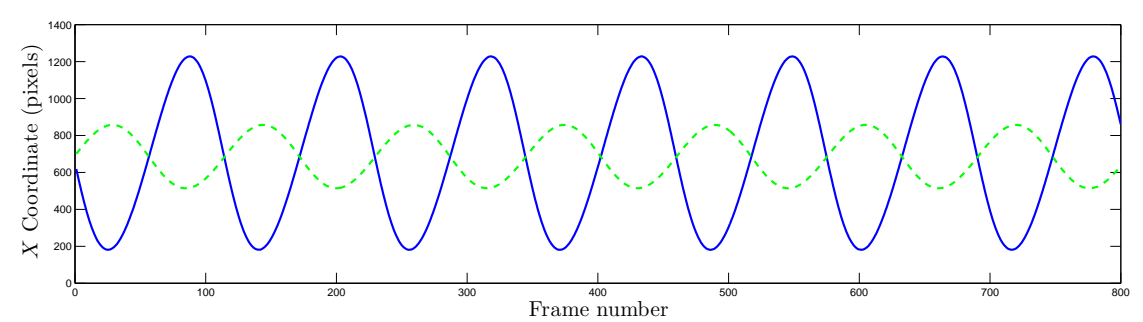

(a) The $x$ coordinate of two tracked targets in the sequence of images

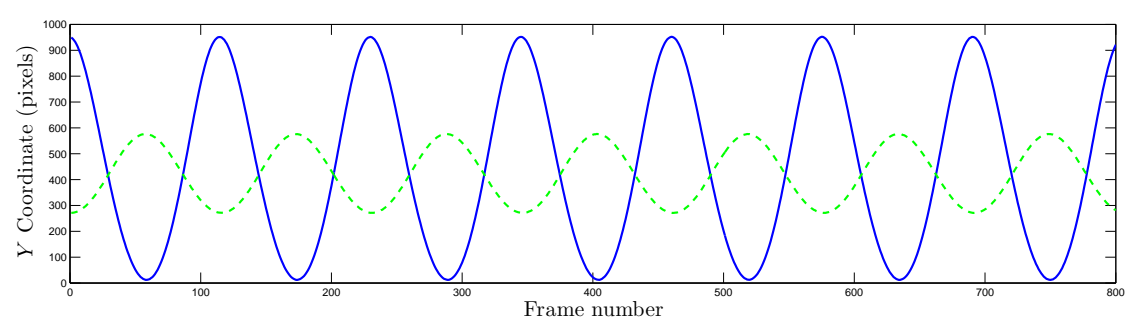

(b) The $y$ coordinate of two tracked targets in the sequence of image

Figure 5.8: The $x$ and $y$ coordinates of the two tracked targets 
In Figure 5.8 the $x$ and $y$ coordinates are plotted of the two tracked targets. Since targets describe an elliptical motion in image space, the $x$ and $y$ coordinates should describe a perfect sine or a cosine function. In case the target was not tracked correctly there would be sudden change or jump in the tracked function. Since the functions in the figure are perfectly smooth and there are no changes in the amplitude, it can be concluded that the targets were tracked and measured correctly.

\subsubsection{Calculated Object Coordinates}

In Section 3.3.6 it was described how object coordinates can be calculated using intersection with DLT. Unfortunately the reference points needed to calculate the DLT parameters were not made available for this study and therefore an alternative approach is used to calculate the object coordinates.

Using the first stereo pair of image of the two sequences a relative orientation is performed using the software PhotoModeler to calculate a relative model. This relative model is then used to calculate DLT parameters. Using the DLT parameters the model coordinates are calculated of all stereo pairs in the sequence. The absolute orientation is performed by an affine transformation, which is calculated from the model coordinates in the first frame and the object coordinates measured using the software PhotoCore. In the next subsections the results of the orientation step will be given.

\section{Relative orientation}

Using the software PhotoModeler a relative orientation was performed between the first frames of the left and right camera. PhotoModeler is a photometric software, which provides image based modeling for accurate measurement and 3D models. It provides possibilities to work with or without camera calibration and modeling in absolute or relative coordinates (PhotoModeler, 2010). The model coordinates obtained from PhotoModeler, by applying a relative orientation, are illustrated in Figure 5.9.

Using the obtained model coordinates from PhotoModeler, DLT parameters were calculated using the equations described in Section 3.3.5. The values of the 11 DLT parameters in the left and right image are given in Table 5.1.

Since the camera's position and orientation do not change during the whole image acquisition process, the obtained DLT parameters can be used for all 800 pairs of images. Using these fixed parameters and the measured model coordinates in a pair of images, the model coordinates of targets can be calculated in the whole sequence. In Figure 5.10 the blue targets are 


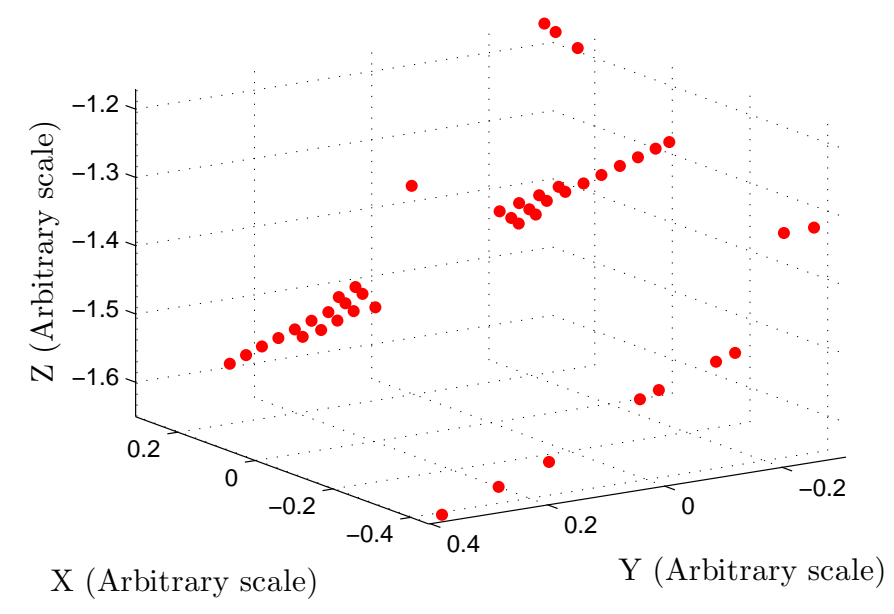

Figure 5.9: Object coordinates calculated with PhotoModeler by applying relative and absolute orientation

Table 5.1: Calculated DLT parameters

\begin{tabular}{crr}
\hline \hline DLT parameters & image1 & image2 \\
\hline$L_{1}$ & 0.9176 & 1.0286 \\
$L_{2}$ & 0.0508 & 0.0182 \\
$L_{3}$ & -0.2147 & 0.1608 \\
$L_{4}$ & 0.1359 & 0.7223 \\
$L_{5}$ & 0.0298 & 0.1426 \\
$L_{6}$ & -0.8946 & -0.9749 \\
$L_{7}$ & -0.2513 & -0.2701 \\
$L_{8}$ & -0.0000 & 0.0133 \\
$L_{9}$ & 0.0000 & 0.0002 \\
$L_{10}$ & 0.0000 & 0.0000 \\
$L_{11}$ & -0.0004 & -0.0005 \\
\hline
\end{tabular}


the calculated object coordinates and the red targets are the targets used to calculate the DLT parameters.

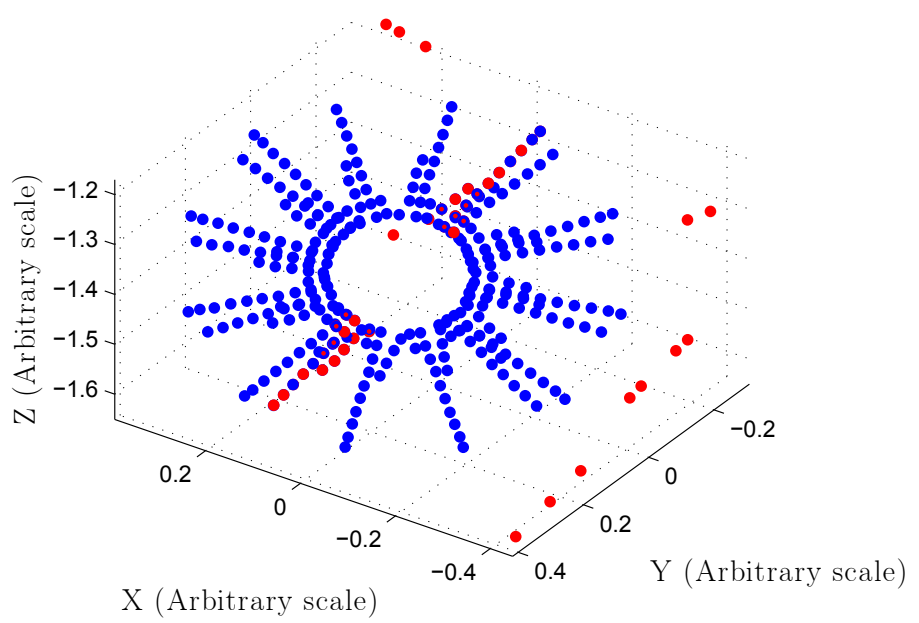

Figure 5.10: Model coordinates calculated of the sequence of images using the DLT parameters

\section{Measuring reference targets}

Using five images of the blade with the coded targets, the software PhotoCore calculated the camera calibration parameters, object coordinates of the coded targets and other detected circular targets. In Figure 5.11 the calculated targets are shown, the dots in red are the twelve coded targets, the dots in green are the sixteen targets from the blade and the dots in blue are circular artifact detected in the background of the image.

\section{Absolute orientation}

The absolute orientation was performed by calculating an affine transformation between the model coordinates and the reference points. This obtained affine transformation was applied to all model coordinates to calculate the object coordinates. The blue dots in Figure 5.12 illustrate the calculated object coordinates of the targets of the wind turbine in several frames and the red dots are the calculated object coordinates from PhotoCore. 


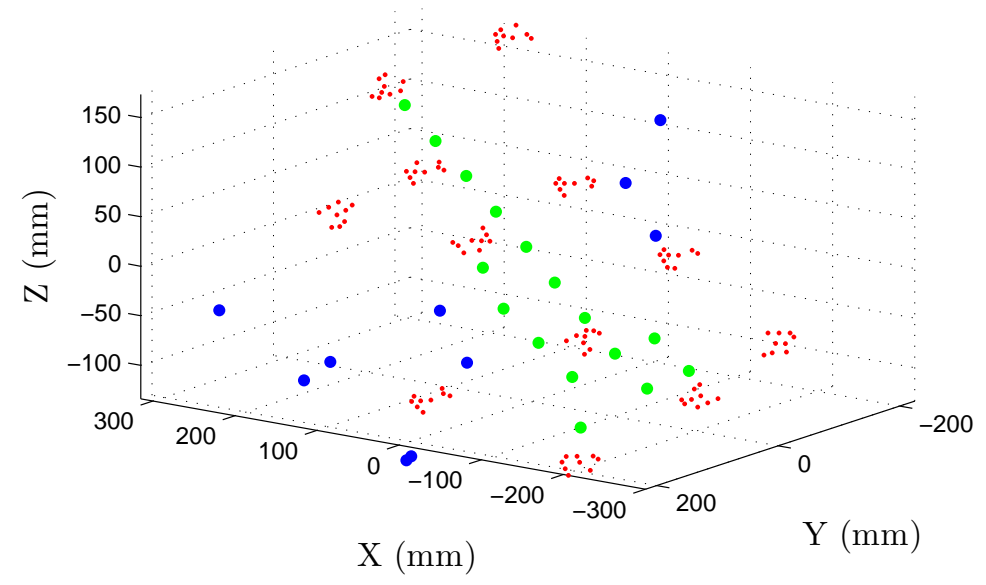

Figure 5.11: Automatically calculated object coordinates of coded and circular targets using the software PhotoCore

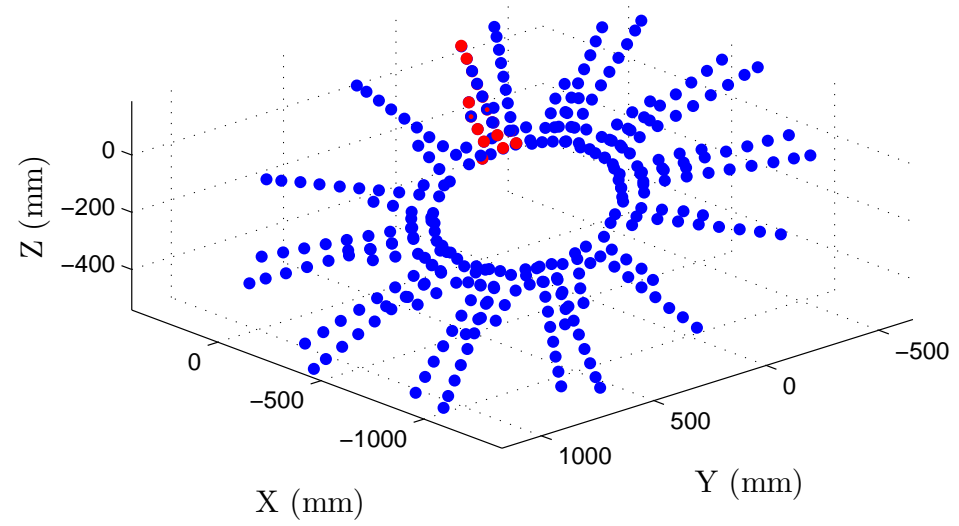

Figure 5.12: Absolutely oriented object coordinates of the targets 


\subsubsection{Results of Vibration Measurement}

Using the PCA described in Section 3.4 the vibrations are derived from the calculated object coordinates of the tracked targets.

In Figure 5.13 the third principal component containing the vibrations of two targets are shown. The magenta line is the vibration of the target with the largest rotational radius and the blue line is the vibration of the target with the smallest rotational radius.

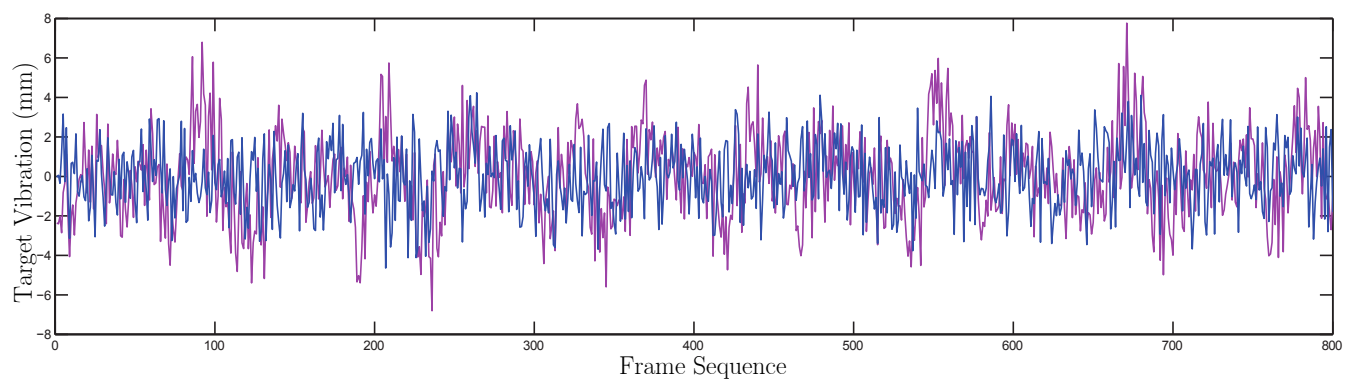

Figure 5.13: The third principal component or derived vibration from the calculated object coordinated of two tracked targets

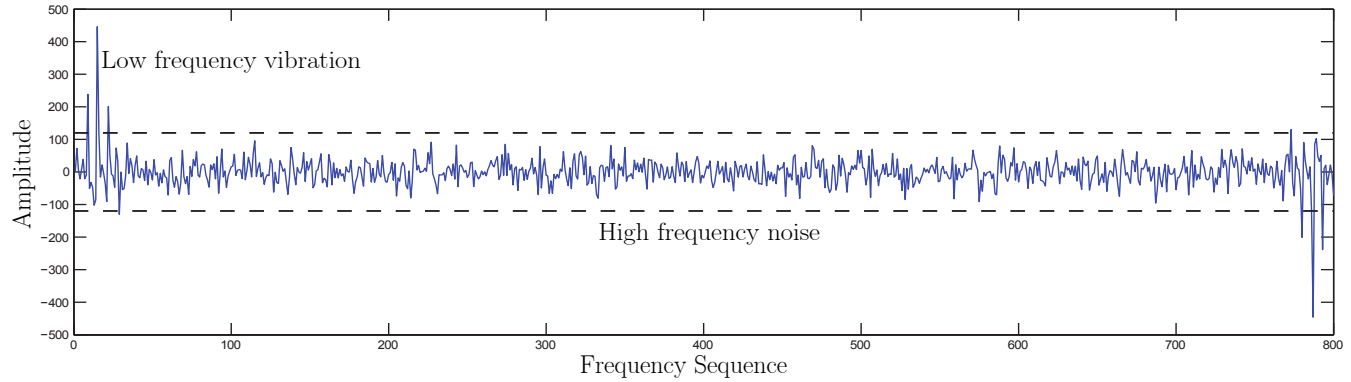

Figure 5.14: Signal vibration transferred from time domain to frequency domain using Fast Fourier transformation

The signal in Figure 5.13 includes besides vibrations also noise of target measurements. To distinguish the vibrations and the noise, the vibration signal can be transformed from time domain to frequency domain using a Fast Fourier transformation. In Figure 5.14 the imaginary component of the signal in frequency domain is given of the magenta vibration signal of Figure 5.13. The low frequencies (high amplitude) are the real vibration of the blades, while the high frequencies (low amplitude) are the measurement noise. 
The noise and the vibration of the signal in Figure 5.14 are distinguished by an absolute amplitude threshold of 120 . From the frequencies outside the noise threshold, it can clearly be seen that there are three different peak or vibrations frequencies. The further processing of these different vibrations are out of the scope of this study. The signal filtered from noise is illustrated in Figure 5.15, were the magenta line is the original signal in time domain and the black line the filtered signal.

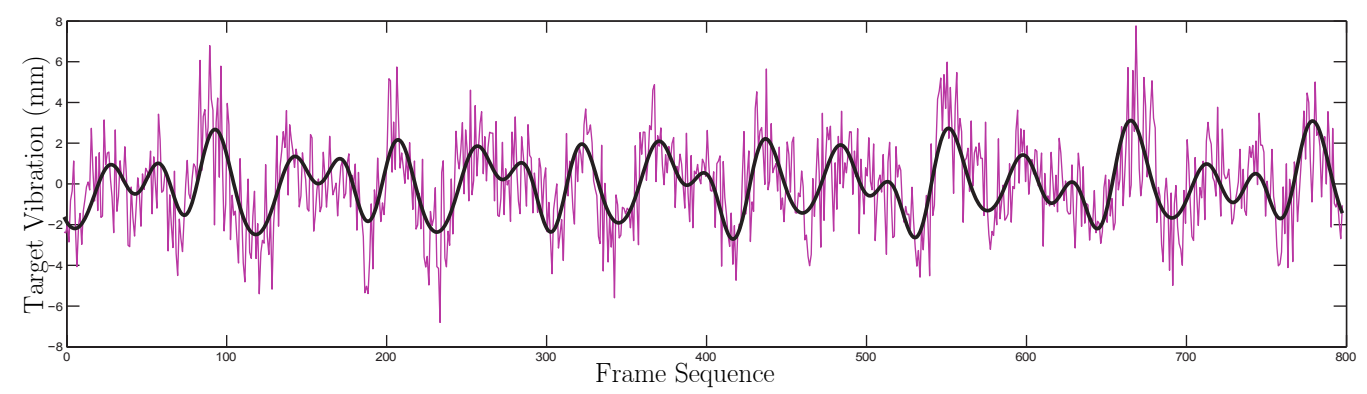

Figure 5.15: Vibration signal filtered with threshold frequency value

The tracks of the two targets in the rotational plane of the first and second principle components are plotted in Figure 5.16. The magenta and the blue lines are the circles fitted to the track of the targets which is plotted in black. The distance between the fitted circle and the track position is illustrated in Figure 5.17.

\subsubsection{Discussion on Measured Vibration}

The track of the two targets would be expected to be a circle in the rotational plane. From the Figures 5.16 and 5.17 it can be noticed that the tracked position of the two targets deviate with respect to the fitted circle. From the deviations it can be concluded that the tracked target with the smallest radius has smaller deviations, while the target with the larger radius has larger deviations. To explain these deviations the following hypothesis are discussed:

- the vibrations of a target will also cause deviations to the $x$ and $y$ coordinates with respect to the ideal rotational circle. This type of deviation is radius dependent, the longer the radius the larger the deviation in the $x$ and $y$ coordinates. This radial dependency can clearly be seen in both fitted circles. The smallest circle has small deviations 


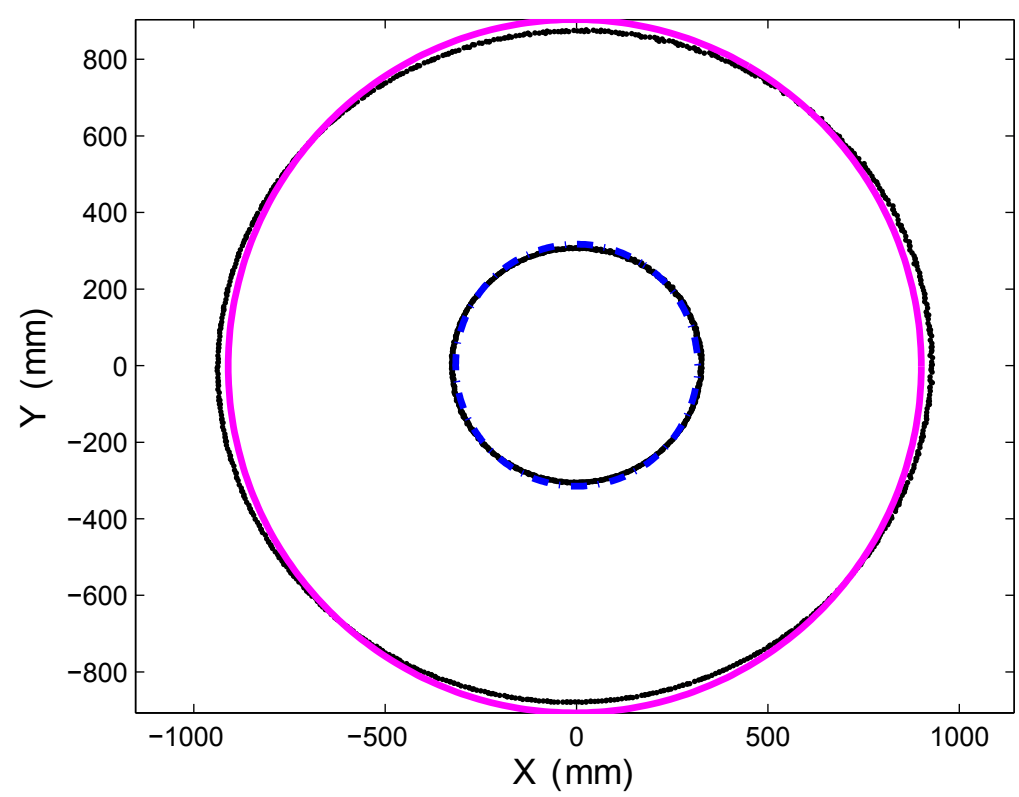

Figure 5.16: Circle fitted to the tracked coordinates of two targets in the rotational plane of the first and second principal component

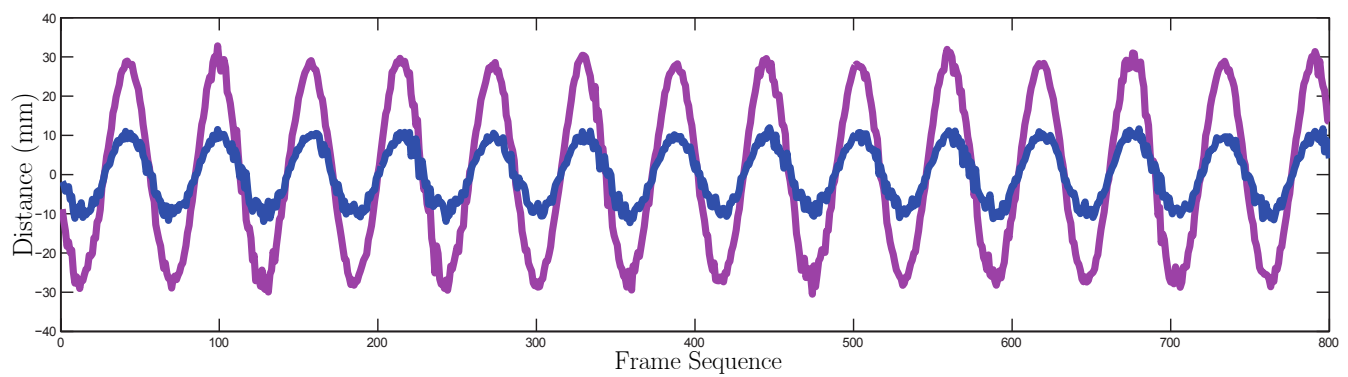

Figure 5.17: Distance between the calculated object coordinates of the two tracked targets and the fitted circle 
while the larger has larger deviations. But the magnitude of the deviations $(35 \mathrm{~mm})$ cannot be explained by the relative small vibration (maximum of $6 \mathrm{~mm}$ ), and therefore this hypothesis is rejected.

- since no corrections for lens distortions were applied, this could also cause errors in the measured image coordinates of the targets. These errors will cause a false parallax to the target position in the stereo pair images. The calculation of the $Z$ coordinate is directly dependent on the parallax of a target in the stereo pair. Parallax differences are the most sensitive along the baseline of the cameras and the least perpendicular to the base line. Not applying lens distortion will lead to errors in the calculated object coordinates, which probably has caused the deviation to some extend.

- the reference targets used for the absolute orientation were not evenly disturbed along the measurement space, which could have caused an improper scaling of the relative model. This improper scaling is probably the most important factor causing the deviation. 


\section{Chapter 6}

\section{Performance Analysis of The Methods}

In this chapter a performance analysis will be performed of the methods used to calculate the vibration of the model wind turbine used in the experiment. In Section 6.1 a robustness analysis will be performed of the developed method to automatically measure and track targets in a sequence of images. An accuracy assessment will be done of the calculated object coordinates in Section 6.2 .

\subsection{Robustness of Automated Target mea- surement}

In Section 5.2.1 the results are described of the automatically measured targets by using the method developed in Chapter 4. To analyze the performance of this proposed method a robustness analysis is performed in this section. The developed method consists of several steps, each of these steps are analyzed individually using the images obtained in the experiment.

\subsubsection{Target detection}

The first step of the automated target measurement in a sequence of images is target detection. To automatically detect the target a histogram image segmentation technique is used. This technique works well as long as the different features in the histogram of the image are clearly distinguishable. In Figure 6.1(a) is an example of one of the images acquired in the experiment and its corresponding histogram is given in Figure 6.1(b). Using the 
histogram of the image, the two main features namely the targets and the background are clearly distinguishable by a histogram threshold of 100 .

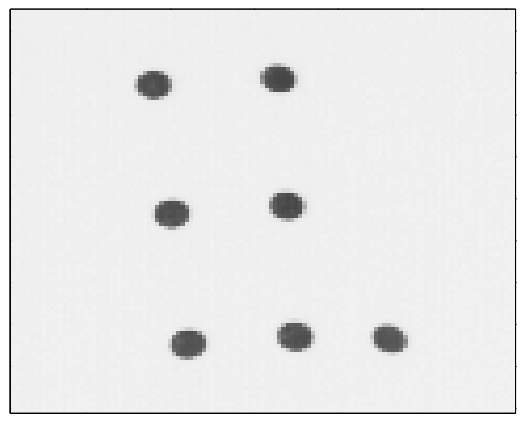

(a) Image without noise

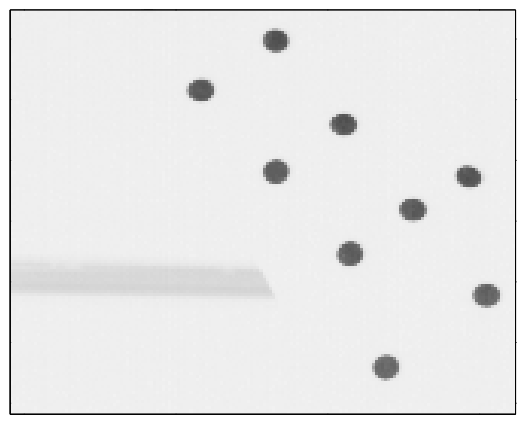

(c) Image with noise

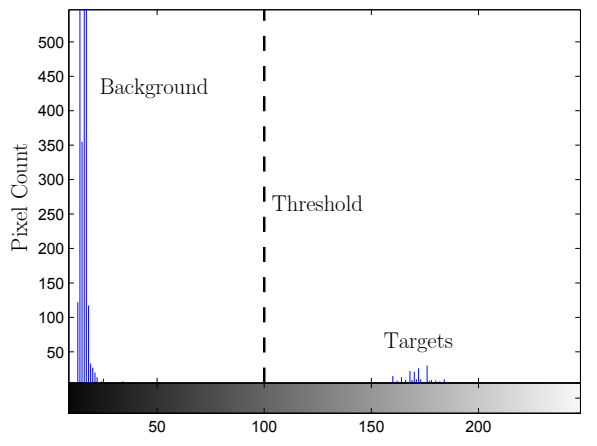

(b) Histogram of image without noise

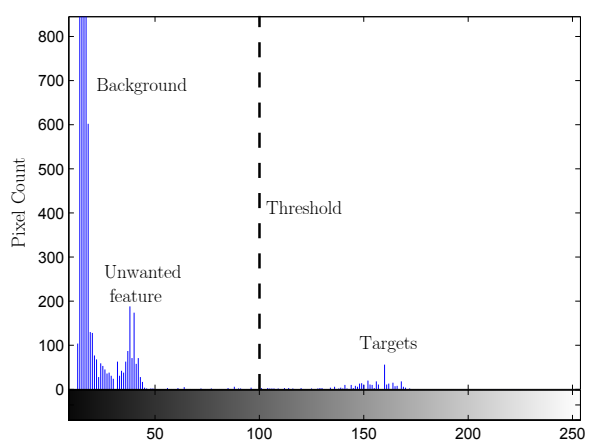

(d) Histogram of image with noise

Figure 6.1: Robustness analysis of threshold value for image segmentation

It might also happen that other features will be visible in an image such as the dark strip in Figure 6.1(c), which was created by a light bulb. In the histogram (Figure 6.1(d)) of this image this third feature can be seen, but still using the threshold of 100 the targets can be detected unambiguously.

Since images made with high speed cameras use short exposure times, only retro-reflective targets illuminated with artificial light will be clearly visible in the image. In case other features such as the light bulb are visible in the image it is a matter of adjusting the threshold to successfully detect the targets. The segmentation is not sensitive to threshold, because there is a large distance between the targets and the background or other unwanted features in the histogram. 


\subsubsection{Removing Reference Targets}

After the targets are successfully detected, the next step is to remove the reference targets which do not need to be tracked in the whole sequence of images. The reference targets are detected by finding targets in the first three frames, which have a difference in position below a threshold.

This threshold value can be chosen empirically and is relatively small compared to the motion of the targets. To determine if it is possible that the motion of a target in successive images will be smaller then the threshold, this was calculated for a range of rotation speeds and image acquisition frequencies (see Figure 6.2). For this calculation the target with the smallest radius was used with respect to the center of the motion in the image.

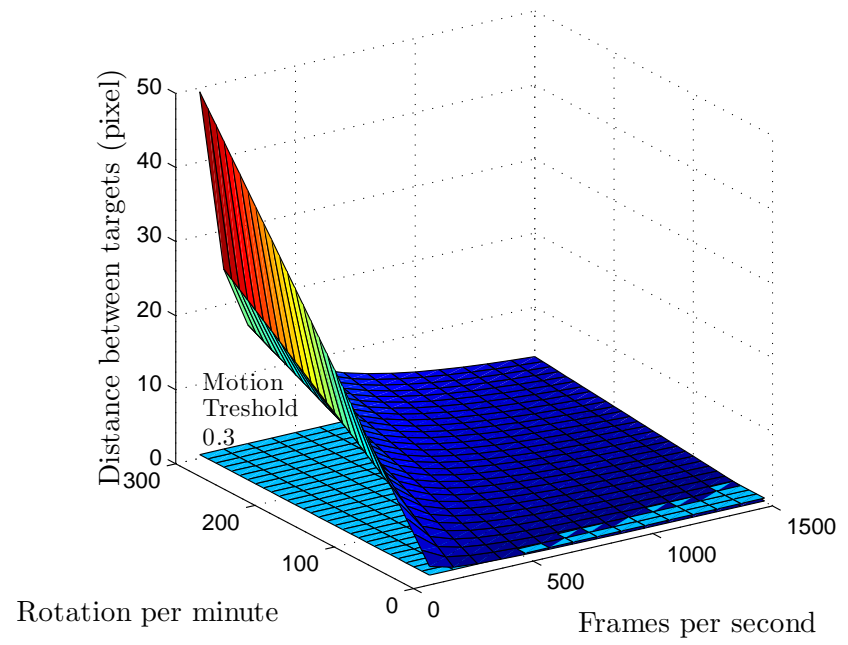

Figure 6.2: The motion of a target calculated for a range of rotation speeds and image acquisition frequencies

Using the images of the experiment, a maximum deviation of 0.3 pixels was measured between positions of the reference target. If this value is compared with the simulated distances of Figure 6.2, it can be concluded that a reference target will be distinguishable from motion targets for all practical combinations of rotation speed and acquisition frequency.

\subsubsection{Establishing Correspondence}

Once the reference targets are removed, the next step is to establish correspondence between the targets of the first three frames. The correspondence 
is established using the four constraints (Section 4.4) equal radius, equal angle, angle $\approx$ mean all angles and closest target to line.

To test the robustness of the constraints these were applied to all three successive frames in the two sequence of images acquired in the experiment. The obtained results were then compared with the tracked results shown in Section 5.2.1. To measure the performance of each constraint this test was repeated again, but every time one constraint was excluded.

In Table 6.1 the results of this test are given. From these results it can be concluded that the first and second constraint contributed for 99.66 $\%$ for the establishing correspondences. This can be explained by the fact that these two constraints define the basic parameters of a circular motion namely radius and rotational angle. The third constraint is used to eliminate ambiguous cases of equal angular motion and is used very few times $(0.33 \%)$. The last constraint is not used at all, but this constraint was primarily added to ensure that one combination is chosen in case if an incorrect combination was not rejected by the previous constraints.

Table 6.1: Performance of the correspondence constraints, where $1=$ equal radius; $2=$ equal angle, $3=$ angle $\approx$ mean all angles and $4=$ closest target to line

\begin{tabular}{cc}
\hline \hline Constraints used & Correct $(\%)$ \\
\hline $1-2-3-4$ & 100 \\
$1-2-3$ & 100 \\
$1-2-4$ & 99.66 \\
$1-3-4$ & 92.33 \\
$2-3-4$ & 73.92 \\
\hline
\end{tabular}

As second test of robustness, the minimum acquisition frequency is determined for which this method to establish correspondence would still have a success rate of $100 \%$. To perform this test the range of acquisition frequencies was simulated by increasing the interval between successive frames. Then using all the constraints the correspondence was established for all three successive frames in the whole sequence of images for every acquisition frequency. To determine the number of incorrect corresponding targets, the obtained results were compared with the tracked results from Section 5.2.1. The number of incorrect corresponding targets of the range of acquisition frequencies is illustrated in Figure 6.3. From Figure 6.3(a) it can be seen that for an interval larger than two, the constraints cannot detect all incorrect correspondences. This interval of two images is equal to an acquisition 
frequency of $125 \mathrm{~Hz}$, which is relatively low for a high speed camera.

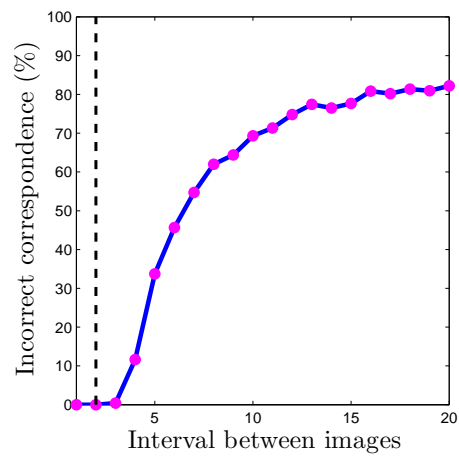

(a) Error rate at various intervals between successive image

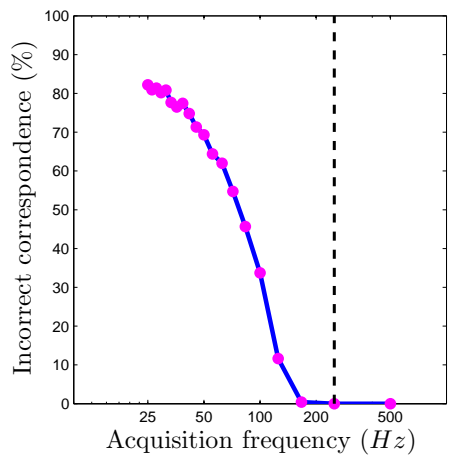

(b) Error rate at a range of acquisition frequencies

Figure 6.3: Robustness analysis of creating correspondence to determine acquisition frequency with acceptable error rate

Since for higher acquisition frequencies the data could not be simulated no test was performed for those frequencies. By using higher acquisition frequencies the motion between frames will become smaller and therefore easier to establish correspondence. In this test the rotational speed of the wind turbine was not varied, but the performed test is equivalent to increasing the rotational speed and keeping the acquisition frequency constant.

\subsubsection{Model Based Target Tracking}

If the position of a target is known in at least three frames, its position in the next frame can be predicted. The robustness of this prediction is analyzed by calculating the difference between the predicted locations and the actual location of the target. This difference is calculated for targets in the whole sequence of images of the experiment and the mean values of it are given in Figure 6.4(a), while the maximum is illustrated in Figure 6.4(b). The largest deviation between the predicted and actual location is 4.3 pixels, which is still smaller than the radius of the target. This means that the predicted position is within the target.

To correct the predicted position a template window is selected to find the target edges. To the detected edges a circle is fitted to precisely determine the target's center. In this experiment a window size of 20 pixels is used, which is twice the size of the target ensuring that the whole target is covered by the window. 


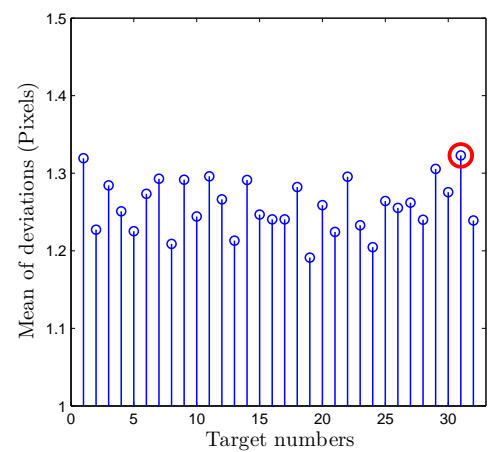

(a) Mean deviation of the predictions per target

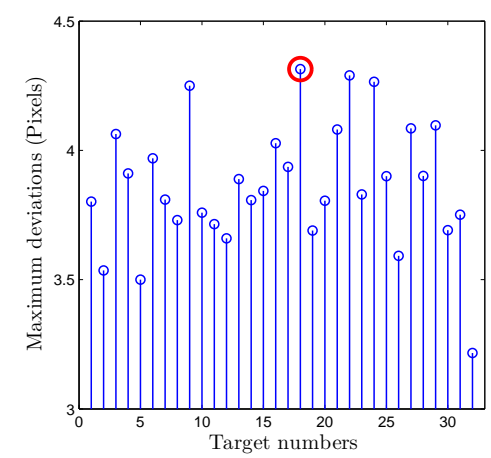

(b) Maximum deviation of the predictions per target

Figure 6.4: The mean and maximum deviations of the predicted location per target

To analyze the performance of the predicted location at a range of acquisition frequencies, the test is repeated whereby the interval between the successive images is increased. Since target 31 (see Figure 6.4(a)) had the largest mean deviation, it is used for this test. The maximum deviation of this target is calculated for a range of intervals and is illustrated in Figure 6.5(a). From this figure it can be concluded that for an interval of 9 images the predicted location is still on the target. For an interval from 9 to 11 images the target will be within the selected window. For intervals larger than 11 images, which is equivalent of $45 \mathrm{~Hz}$, the target will not be in the selected window and therefore will not be detected.

\subsubsection{Precise Target Measurement}

After the target positions are predicted, a window is selected around it to detect the edges of the target. A circle is fitted to these edges to precisely pin-point the center of the target. Since the edges are detected within the selected window, it is important that the window is large enough to cover the whole target and small enough that it does not contain other targets.

To analyze the optimum size of the template window a test is performed where the size of the window is varied and the number of incorrect target centers are measured. The results of this test are illustrated in Figure 6.6(a), where for the blue line no outlier filtering was done while for the dotted magenta line RANSAC was used.

From this figure it can be concluded that the window size should be at 


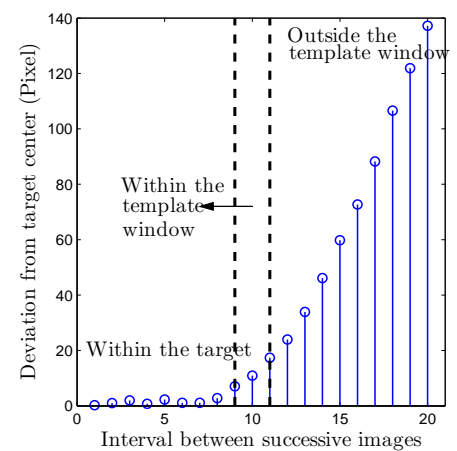

(a) Quality of target prediction at various intervals between successive image

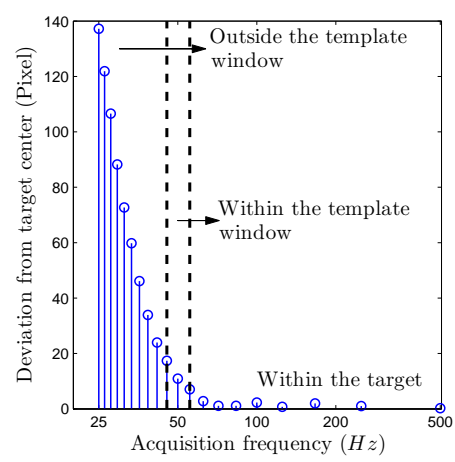

(b) Quality of target prediction at a range of acquisition frequencies

Figure 6.5: Robustness analysis of target prediction to determine acquisition frequency with acceptable predictions

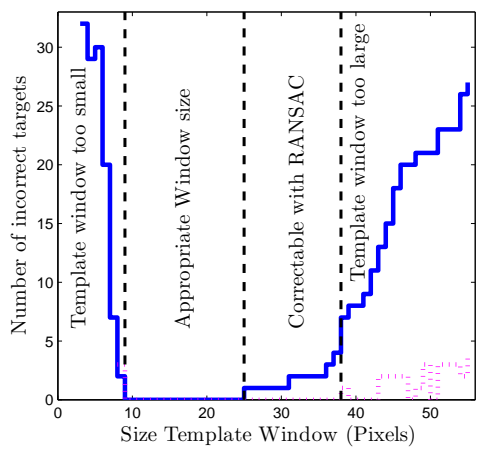

(a) Uncorrected edges detected at a range of window sizes

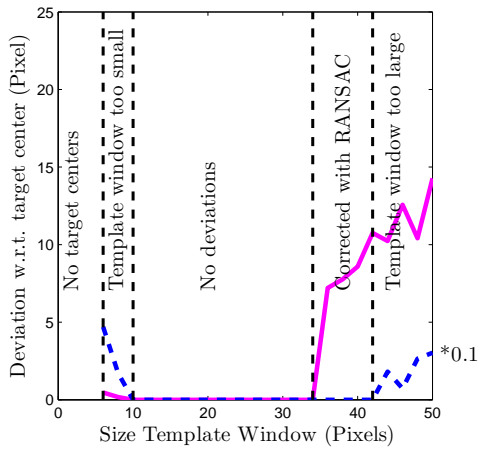

(b) Deviation of target centers at a range of window sizes

Figure 6.6: Robustness analysis to determine the range of acceptable template window sizes 
least larger than 10 pixels, which is the same as the target size. The maximum window size without any outlier filtering is 25 pixels, which corresponds with the distance between the two closest targets (see Figure 6.7). If RANSAC is used the largest window size is 38 pixels, which corresponds with the performance of RANSAC which can remove outliers up to $50 \%$.

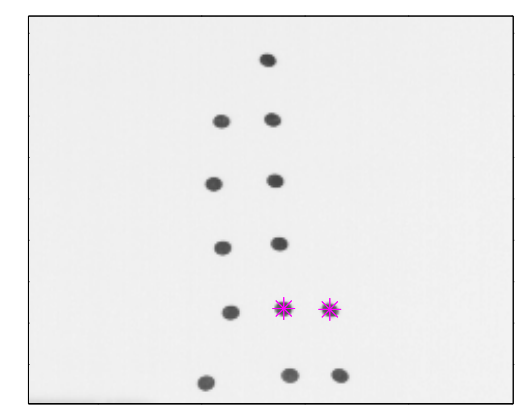

Figure 6.7: The two closest targets identified to analyze the template window size

\subsubsection{Comparison of Circle Versus Ellipse Fitting}

To measure the target center with subpixel precision a test was proposed in Section 4.6.4 to determine the difference between fitting a LSQ circle or ellipse to pin-point the target center. In the test four sets of edge points were generated along an ellipse and a LSQ circle and an ellipse were fitted (see Figure 6.8). The difference between the centers of both fitted models is given in Table 6.2 .

Table 6.2: Results of difference between centers of a LSQ fitted circle and ellipse

\begin{tabular}{lcc}
\hline \hline Number of points & Difference in $\mathrm{x}$ & Difference in $\mathrm{y}$ \\
\hline 5 random & 1.25 & 1.33 \\
360 & 0 & 0 \\
20 random & 0.31 & 1.22 \\
20 evenly distributed & $-1.7 e^{-14}$ & $-1.4 e^{-14}$ \\
\hline
\end{tabular}


From the result in Table 6.2 the following can be concluded of using an circle or ellipse to pin-point a target center:

1. if there are many edge points (Figure 6.8(b)) there is no difference;

2. if there are less points as in Figure 6.8(a) and 6.8(d) there is some difference;

3. but as the number of points increases the difference decreases;

4. and if there are less points, but evenly distributed (Figure 6.8(d)) the difference is almost zero.

Since the edge points of a target are always evenly distributed, a circle is sufficient to pin-point the target center. A practical advantage of using a circle over an ellipse is, that the mathematically function of a circle is simpler. For a circle three parameter are needed to be estimated, while for an ellipse 5 parameter are needed to be estimated.

\subsubsection{Performance Target Tracking}

The final step of the robustness analysis is to determine if the tracked positions of a target are correct. Here the knowledge is used that a target in object space describes a circular motion. In image space this circular motion is deformed to an ellipse due to perspective distortions. So by fitting an ellipse to the tracked positions, it can be determined if a target was tracked correctly. In Figure 6.9(a) an example is given of two tracked targets for which an ellipse is fitted.

By measuring the deviations between the tracked position and the fitted ellipse, it is possible to detect if a target is tracked correctly or a single position is measured incorrectly. If the deviation between the measured positions and the fitted ellipse is too large, it can be concluded that the measured positions are incorrect.

In Figure 6.9(b) a histogram is given of the measured deviation between the tracked positions of all targets and the fitted ellipses. The maximum deviation between the measured position and the fitted ellipse is below one pixel. This value is relatively small and therefore it can be concluded that all targets were tracked correctly.

\subsection{Accuracy Assessment of the Results}

In this section an accuracy assessment is done of the target measurement in the images and the three steps undertaken to calculate the object coordinates 


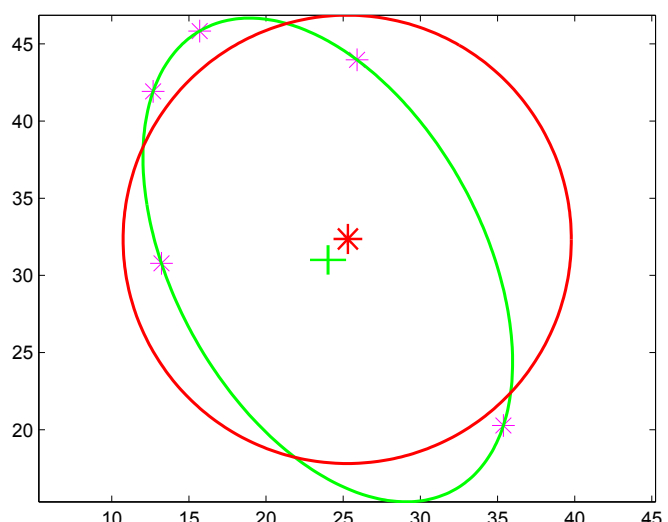

(a) 5 random points used

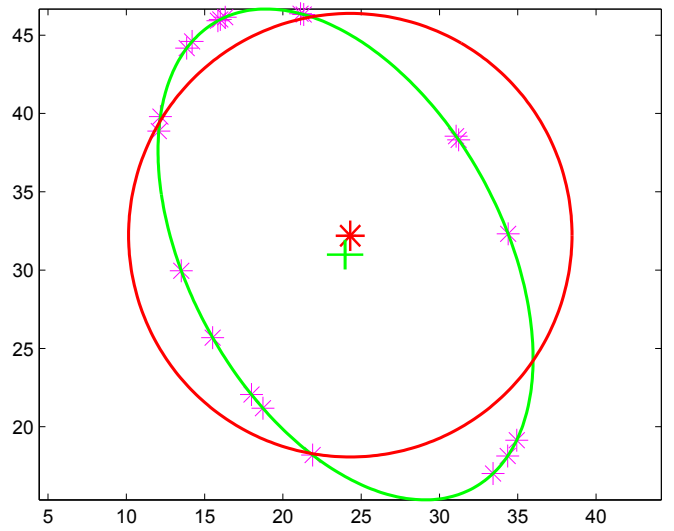

(c) 20 random points used

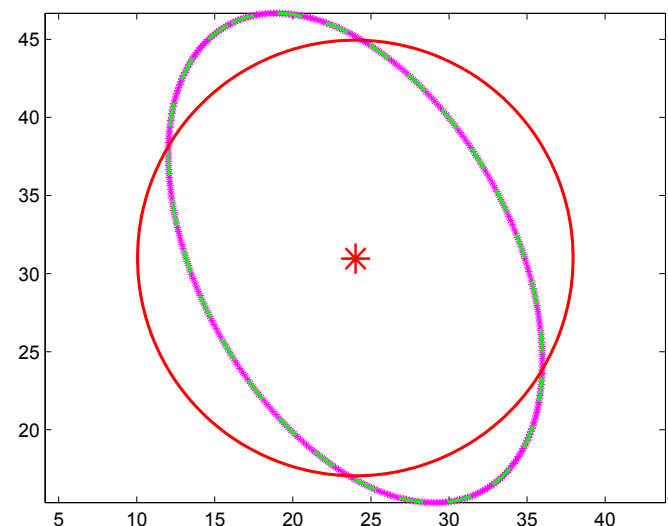

(b) 360 points used

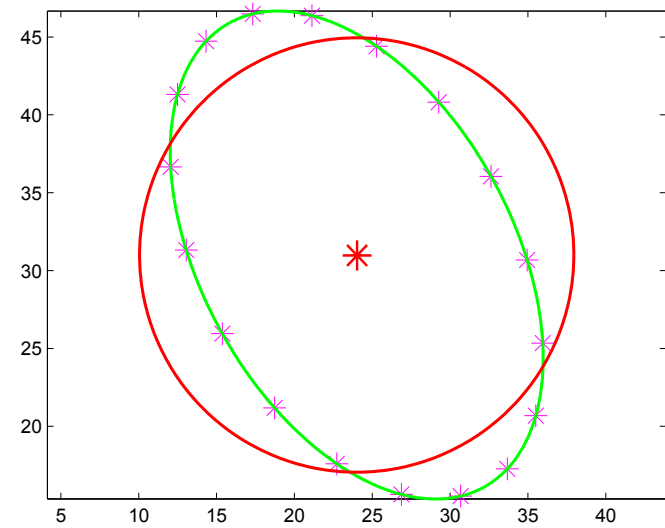

(d) 20 regular space points used

Figure 6.8: Fitting an ellipse and a circle to a set of points to analyze the difference between the measured target centers 


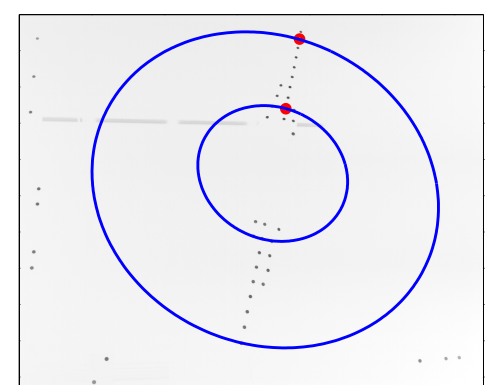

(a) Ellipse fitted to the track of a target

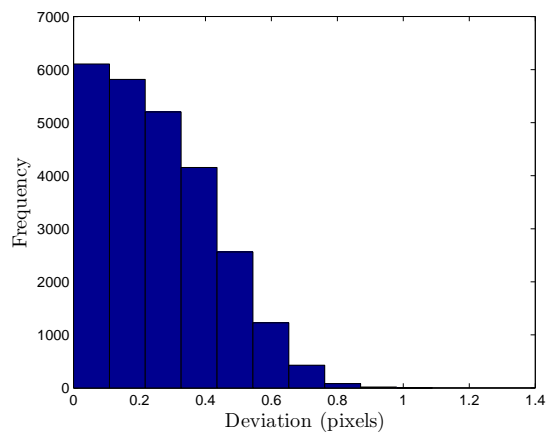

(b) Deviation between fitted ellipse and target positions

Figure 6.9: Analyzing the correctness of the tracked target by fitting an ellipse

of the targets placed on the wind turbine. The accuracy of the target measurement will be described in Section 6.2.1. In Section 6.2.2 the residuals of the relative orientation are described. The accuracy of the reference targets measured with PhotoCore will be given in Section 6.2.3. In Section 6.2.4 the residuals of the absolute orientation are given.

\subsubsection{Accuracy of Target Measurement}

In Section 4.7 a test was described to determine the accuracy by which the center of a target can be pin-pointed using an artificial target. The artificial target was created in a high resolution image of 1000 by 1000 pixels. The value of 100 Pixels were assigned to pixels inside the targets and for pixels outside the target the value of 14 was assigned, which is approximately the mean pixel value of the background in the images acquired in the experiment. The high resolution image was down sampled to a size of 100 by 100, so that the artificial target had a dimension of 8 by 12 pixels, which is comparable with the size of targets in the images of the experiment. Gaussian noise was added with a mean of zero and standard deviation of three pixels. A point spread function was added with a radius of 2 pixels and motion blur was added with a motion of 3 pixels. In Figure 6.10(a) an example is given of the artificial image. The down sampled image with random Gaussian noise, motion blur and point spread function is illustrated in Figure 6.10(b).

The green dot in Figure 6.10(b) is the actual known position of the target center. The red dots are the detected edges and the blue dot is the center 


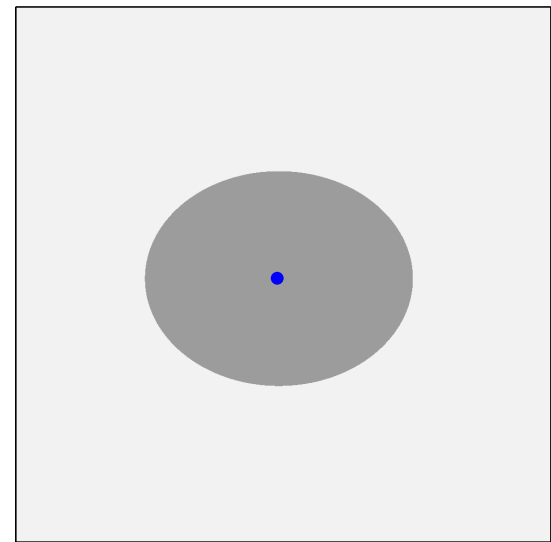

(a) Artificial target created with an image resolution of 1000 by 1000 pixels

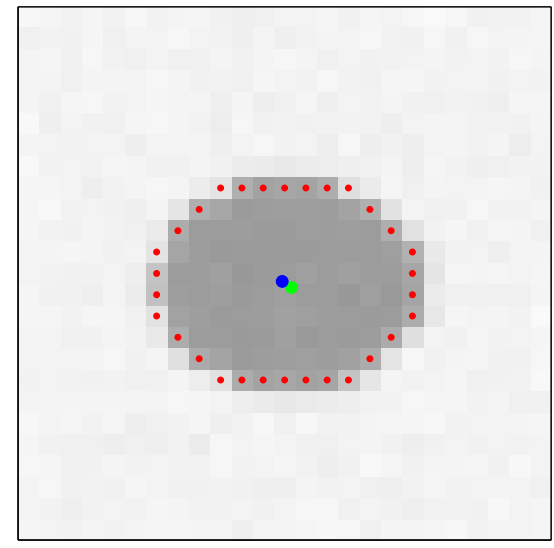

(b) Image resized to a resolution of 100 by 100 with added random Gaussian noise, motion blur and point spread function

Figure 6.10: Artificial target created to estimate the accuracy by which the center of a target can be pin-pointed

of the fitted target. To get a representative estimation of the accuracy of the pin-pointed target center the artificial target is generated 1000 times. The mean and standard deviation of the differences between the pin-pointed and actual target center are given in Table 6.3. From these values it can be concluded that the center of a target can be pin-pointed with an accuracy of 0.25 pixels.

Table 6.3: Mean and standard deviation of the differences between the measured and actual position of an artificial target

\begin{tabular}{lcc}
\hline \hline & Mean (pixel) & Std(pixel) \\
\hline Dif $x$ & -0.2348 & -0.0634 \\
Dif $y$ & 0.0574 & 0.0905 \\
\hline
\end{tabular}

To determine the size of the measured error in object space the scale of the image is determined using the measured reference point. From the scale the size of one pixel is determined in object space, which is $2 \mathrm{~mm}$. This mean that the measurement in image space has an accuracy of $0.5 \mathrm{~mm}$ in object space. 


\subsubsection{Accuracy of Relative Orientation}

By means of a relative orientation model coordinates were calculated of the targets. The relative orientation is obtained by applying the co-planarity constraint. This constraint implies that the position of a target in object space and it's position projected in two or more images lie on a plane or are co-planer (Hartley and Zisserman, 2004). Due to measurement errors the positions of the targets measured in image space have to be adjusted to satisfy the co-planarity condition. The difference between the measured and adjusted positions are the residuals per measurement in image space and are illustrated in Figure 6.11. In Table 6.4 the mean and standard deviation of the residuals in the left and right image are given.

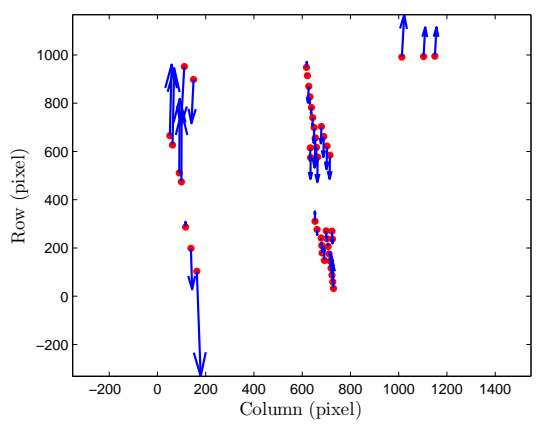

(a) Residual vectors left image

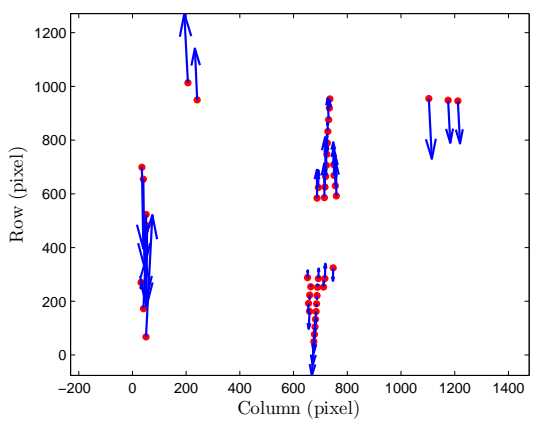

(b) Residual vectors right image

Figure 6.11: Residuals in image space after relative orientation

Table 6.4: Residual vectors in image space obtained by relative orientation

\begin{tabular}{lcc}
\hline \hline & Mean $($ pixel $)$ & Std (pixel) \\
\hline Res x left image & 0.0000 & 0.0001 \\
Res y left image & 0.0049 & 0.1456 \\
Res x right image & 0.0000 & -0.0001 \\
Res y right image & 0.0049 & 0.1348 \\
\hline
\end{tabular}

If the residuals are examined, it can be noticed that the $x$ components of the residuals are close to zero, while the $y$ components are relatively large. This can be explained by the fact that in the adjustment process four measurements are available (two per image), while three unknown $(X, Y, Z$ coordinates) need to be solved resulting in one degree of freedom. This one 
degree of freedom is perpendicular to the epipolar line ${ }^{1}$ resulting in measured coordinates which can only be adjusted in one direction. Since there was no rotation between the two camera's positions, the epipolar line between the two images is parallel with the $x$ coordinates of the images and no corrections are applied to $x$ coordinates.

The overall quality of the relative orientation can be analyzed by the size of the residuals. From Figure 6.11 it can be seen that the targets closer to the image center have smaller residuals than the targets further away. This can be explained by lens distortions which create larger errors away from the image center. From Table 6.4 it can be noticed that the residuals are relatively small and are in the order of one tenth of a pixel. It can therefore be concluded that the relative orientation was performed correctly.

From the obtained model coordinates, DLT parameters were calculated for the left and right image. The DLT parameters are calculated in a LSQ adjustment and therefore residual values are obtained for every image. The vectors of the residuals in the left and right image are illustrated in Figure 6.12 and the mean and standard deviation are given in Table 6.5.

The model coordinates from the relative orientation were also used as observations to estimate the DLT parameters. Therefore the model coordinates were also adjusted. The residual vectors in the model coordinates are illustrated in Figure 6.13. The mean and standard deviation are given in Table 6.6.

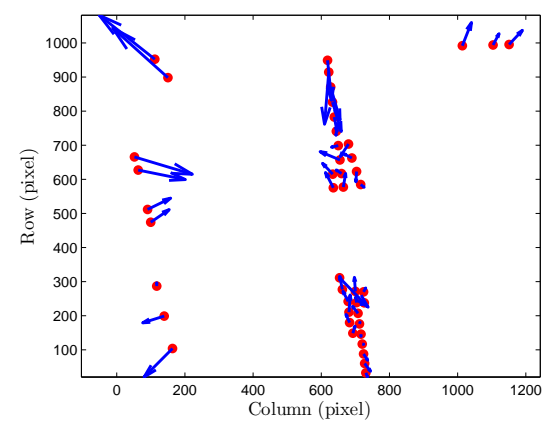

(a) Residuals in the left image

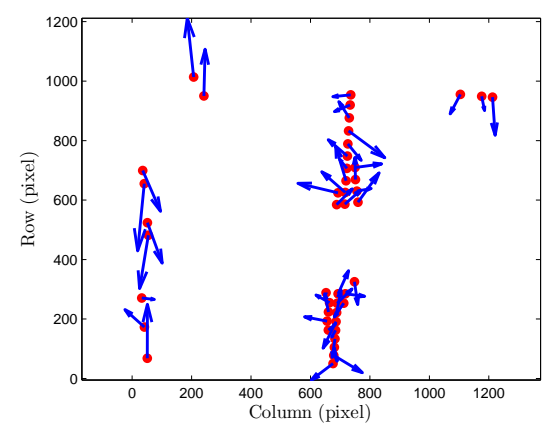

(b) Residuals in the right image

Figure 6.12: Residuals in image space for the calculated DLT parameters

\footnotetext{
${ }^{1}$ The epipolar line is the intersection between the epipolar plane and the image plane going through the projected position of a target in the image plane (Hartley and Zisserman, 2004)
} 
Table 6.5: Statistics of residuals in image space for the calculated DLT parameters

\begin{tabular}{lcc}
\hline \hline & Mean (pixel) & Std (pixel) \\
\hline Res x left image & 0.0002 & 0.0002 \\
Res y left image & 0.3085 & 0.3092 \\
Res x right image & 0.0000 & -0.0001 \\
Res y right image & 0.0947 & 0.1576 \\
\hline
\end{tabular}

From the Figure 6.12 and 6.13 it can be seen that the error vectors point to different directions. This indicates that there are no systematic errors in the estimated model. The error increases away from the image center, which can be explained by the error propagation of the lens distortions. The residual values in image space (Table 6.5) are still relatively small and are in the order of one tenth, but compared to values obtained in the relative orientation these are larger. This increase in error is caused by the error propagation. The residual values in model space (Table 6.6) have no scale and therefore the magnitude can not be analyzed. The mean of the residuals are close to zero indicating that on average the errors are low.

Since these residuals are relatively small and the error vectors are not systematic, it can be concluded that the calculated DLT parameters have an acceptable accuracy.

Table 6.6: Statistics of residuals in model space for the calculated of DLT parameters

\begin{tabular}{lcc}
\hline \hline & Mean (Arbitrary scale) & Std (Arbitrary scale) \\
\hline Res X & 0.0023 & 0.1450 \\
Res Y & -0.0002 & 0.1224 \\
Res Z & 0.0026 & 0.5275 \\
\hline
\end{tabular}

\subsubsection{Accuracy of Measured Reference Points}

To measure the reference targets the software PhotoCore was used. This software uses coded targets with a known dimension as reference. For every calculated object coordinate this software gives an error value. In Figure 6.14(a) the error vectors of the coded targets are illustrated. The error vectors 


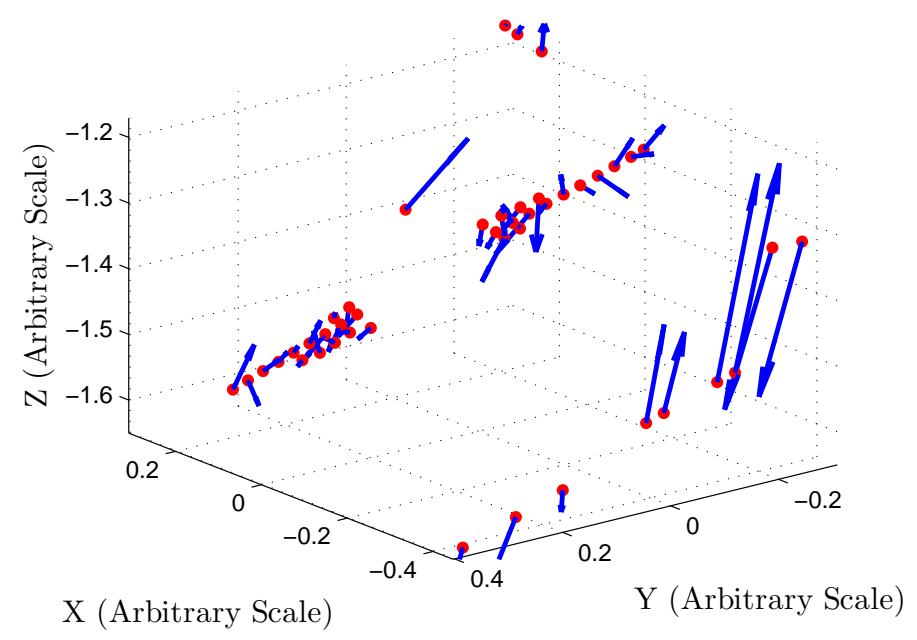

Figure 6.13: Residuals in model space for the calculated DLT parameters

of the targets measured on the blade are given in Figure 6.14(b). The mean and standard deviation of the errors of the measured coordinates are given in Table 6.7.

Table 6.7: Statistics of residuals in image space using PhotoCore

\begin{tabular}{lcc}
\hline \hline & Mean $(\mathrm{mm})$ & Std $(\mathrm{mm})$ \\
\hline Res x coded & 0.0228 & 0.0095 \\
Res y coded & 0.0196 & 0.0053 \\
Res z coded & 0.0198 & 0.0028 \\
Res x detected & 0.0194 & 0.0084 \\
Res y detected & 0.0163 & 0.0036 \\
Res z detected & 0.0187 & 0.0012
\end{tabular}

From the mean and standard deviations in Table 6.7 it can be noticed that these are very small and are in the order of one hundredth of a millimeter. Since the goal in this study is to measure the vibration with millimeter precision, it can be concluded that these errors are relatively small and can be practically neglected. 


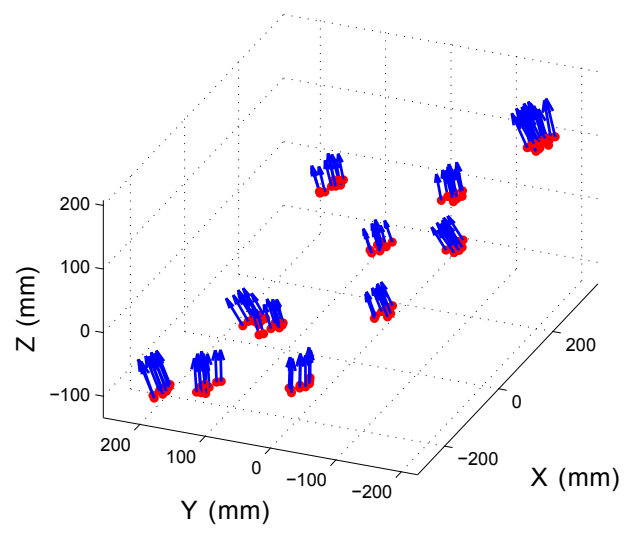

(a) Error vectors of coded target

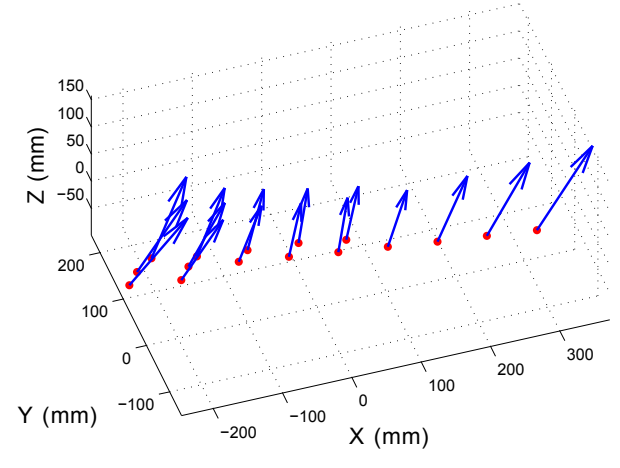

(b) Residual vectors of the detected targets on the blade

Figure 6.14: Residual vectors of the reconstructed targets using PhotoCore

\subsubsection{Accuracy of 3D Reconstructed Target Coordi- nates}

The absolute orientation was performed by calculating an affine transformation between the model coordinates and the measured reference targets. To measure the quality of the affine transformation the model coordinates, used as observation to estimate the model, were transformed to object space and compared to the reference targets. The deviation (residuals) between these two sets of coordinates is illustrated in Figure 6.15. The mean and standard deviation of the residuals are given in Table 6.8.

Table 6.8: Statistics of residuals in object space using affine transformation

\begin{tabular}{lcc}
\hline \hline & Mean $(\mathrm{mm})$ & Std $(\mathrm{mm})$ \\
\hline Deviation in X & 0.0000 & 1.0189 \\
Deviation in Y & 0.0000 & 0.4715 \\
Deviation in Z & 0.0000 & 0.6988
\end{tabular}

It can be noticed that the majority of the residual vectors in Figure 6.15 are pointing in one direction. This can be explained by the improper scaling of the model coordinates. The mean values in Table 6.8 are zero. This indicates that the estimated model used for the affine transformation has a good fit between both coordinate systems. The accuracy can be derived from the standard deviation, which indicates the difference between the fitted 


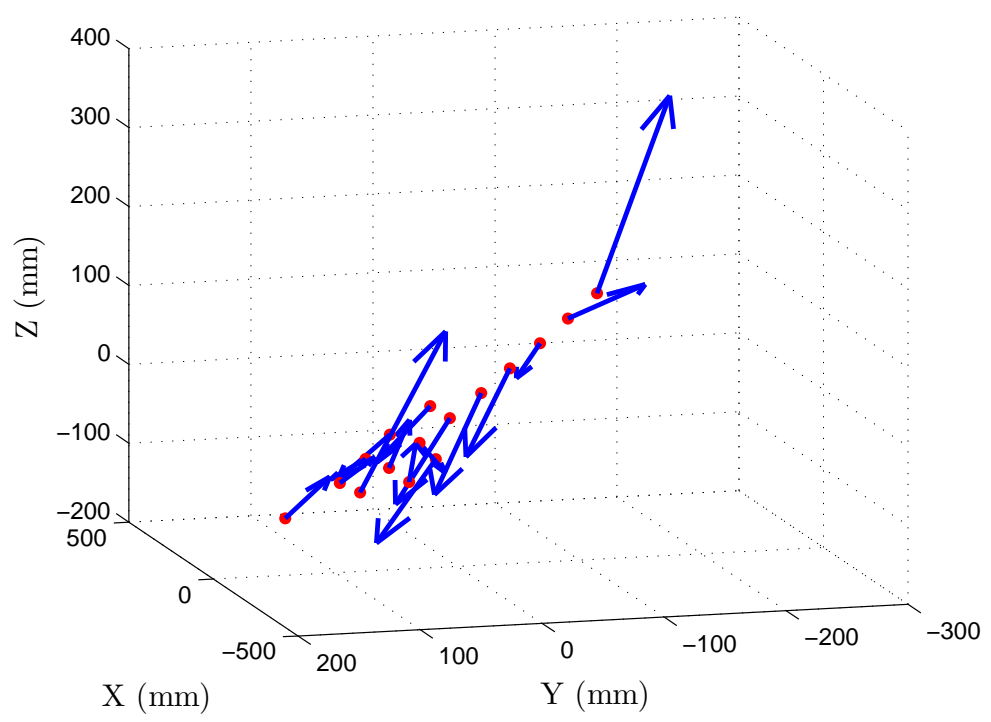

Figure 6.15: Residuals of absolute orientation

model and the used observation.

Since too many steps are involved to calculate the final object coordinates, it is difficult to calculate an accuracy. Instead by calculating the residuals at each step it can be analyzed if the results are acceptable and no errors were made in the estimation model. As final measure of accuracy the sum of the vectors of standard deviation can be calculated, which is $1.32 \mathrm{~mm}$ for the calculated object coordinates.

\subsection{Accuracy of derived Vibrations}

To calculate the accuracy in the vibration direction the obtained residual from the calculated object coordinates can be transformed to the principal components as described in Section 3.4. The mean and standard deviation of the residuals transformed to the three principal components are given in Table 6.9.

From this table it can be concluded that the vibration measured in this experiment has an accuracy of $0.58 \mathrm{~mm}$. It should be kept in mind that this accuracy is calculated using reference points which were not measured in the original experiment setup. The reference point were also not evenly distributed over the measurement area which also degrades the accuracy. No correction for lens distortion were applied for the target measured in the 
Table 6.9: Statistics of transformed residuals to the three principal components

\begin{tabular}{lcc}
\hline \hline & Mean $(\mathrm{mm})$ & Std $(\mathrm{mm})$ \\
\hline Principal component 1 & 0.0008 & 0.1963 \\
Principal component 2 & -0.0008 & 0.8465 \\
Principal component 3 & 0.0000 & 0.5774
\end{tabular}

images, which also reduced the final accuracy. 
Chapter 6. Performance Analysis of The Methods 


\section{Chapter 7}

\section{Conclusions and Recommendations}

In this chapter the conclusions for this study will be presented in Section 7.1 and the recommendations for future work will be given in Section 7.2.

\subsection{Conclusions}

In this study it has been shown that it is possible to automate the process of the vibration measurement of a wind turbine using high speed photogrammetry. To achieve this goal four research question were formulated. The conclusions for each research question are given as follows:

1. The targets in the images can be measured by detecting the edges of the targets and fitting a circle to these edges. From the experiments it has been shown that a target can be measured with an accuracy of 0.25 pixels.

2. The position of a target can be tracked in a sequence of images. Using the knowledge of the circular motion of the wind turbine the targets could be tracked successfully using a circle as model. From a robustness analysis for of the target tracking it could be concluded, that the target tracking is reliable for acquisition frequencies larger then $125 \mathrm{~Hz}$.

3. The automated target detection and tracking is influenced by threshold values used for the histogram threshold image segmentation and creating correspondence. The precise target measurement is influenced by the size of the window to detect edges of a target. To pin-point the target center, a circle can be fitted to evenly distribute target edges. 
4. The target in the images acquired in the experiment can be measured with an accuracy of $0.5 \mathrm{~mm}$ in object space. The accuracy of the calculated object coordinates are $1.32 \mathrm{~mm}$ and the accuracy of the derived vibration is $0.58 \mathrm{~mm}$.

From the performed experiment with the model wind turbine, it can be concluded that the blade vibrations can be measured using high speed photogrammetry.

\subsection{Recommendations}

Based on this study the following recommendations can be made for future work:

- In the developed method for target tracking correspondence was created between the targets of the first three frames using four physical constrains of a circular motion. Instead of these physical constraints also mathematical constraints of a circular motion can be used. The track of the $x$ and $y$ coordinates of a target describes a goniometrical function in image space. Constraints of this goniometrical function could be used to create correspondence between the targets of the first three frames instead of the four constraints.

- To improve the accuracy of the calculated object coordinates, corrections for lens distortion should be applied. Since it has been concluded that DLT is suitable for this application, a self calibration DLT is recommended. The self calibrating DLT contains five extra parameters from which three are for optical distortion and two are for centering distortion. To solve this model at least eight reference points are needed, which is still not too much for such an application.

- To measure the target precisely in the images an edge detection was performed and a circle was fitted to the detected edges. Since the sensitivity of the edge detector is directly related to the accuracy of the target measurement it is necessary to explore other edge detection methods e.g. Sobel or Previt edge detector to determine if the accuracy can be improved.

- Because the lack of reference points an improper error estimation was done of the measured vibration. To determine the actual capabilities of photogrammetry to measure vibrations a detailed error analysis should be carried out using proper reference points. 
- In this study one type of vibration was derived from the tracked target positions. In further studies it should be investigated how the currently used method to derive the flapwise vibrations could be improved and how other types of vibration can be derived from the tracked target positions.

- The developed method for measuring the vibration of a wind turbine was tested on a model wind turbine placed in a wind tunnel. It should be investigated if this method can be extended to measure vibrations of a full scale wind turbine in open air. 
Chapter 7. Conclusions and Recommendations 


\section{Bibliography}

Ariyawansa, D. and Clarke, T. A. (1997), 'High speed correspondence for object recognition and tracking', Videometrics $V$ 3174(1), 70-79.

URL: http://link.aip.org/link/?PSI/3174/70/1

Atkinson, K. (1996), Close Range Photogrammetry and Machine Vision, Whittles Publishing.

Balch, K. S. (2004), Replacing 16 mm Film Cameras with High Definition Digital Cameras, Eastman Kodak Company, San Diego, California.

Britannica (2010), 'www.britannica.com'. visited on: 12-10-2010.

Brush, C. F. (2008), 'A wind energy'.

URL: http://www.windpower.org/en/pictures/brush.htm

Bullock, R. (2006), 'Least squares circle fit'.

URL: http://www.dtcenter.org/met/users/docs/write_ups/circle_fit.pdf

Burner, Tianshu, Burner, A. W. and Liu, T. (2001), 'Videogrammetric model deformation measurement technique', Journal of Aircraft 38, 745-754.

Ceasar, T. and Michaelis, M. (1997), 'Ein neues verfahren zur robusten erkenning textcodierter marker', Zeitschrift fur Photogrammetrie und Fernerkundung pp. 150-157.

Corten, G. and Sabel, J. (1995), 'Optical motion analysis of wind turbines'. Technical Report, SV Research Group, Delft University of Technology.

energy world.com, R. (2009), 'Wind power'.

URL: http://www.renewableenergyworld.com/rea/tech/windpower

Figueroa, F., Mahajan, A., Sayeh, M. and Regez, B. (2007), 'Fiber-optic strain gauge with high resolution and update rate', Technical Briefs.

URL: http://www.techbriefs.com/component/content/article/2027 
Fischler, M. A. and Bolles, R. C. (1981), 'Random sample consensus: A paradigm for model fitting with applications to image analysis and automated cartography', Comm. of the ACM 24, 281-395.

Furnee, E. H., Jobbagy, A., Sabel, J. C., Van Veenendaal, H. L. J. and Martin, F.and Andriessen, D. C. W. G. (1997), 'Marker-referred movement measurement with grey-scale coordinate extraction for high-resolution realtime $3 \mathrm{~d}$ at $100 \mathrm{hz}$, PROCEEDINGS-SPIE THE INTERNATIONAL SOCIETY FOR OPTICAL ENGINEERING 3173, 357-369.

Goldman, M. (1996), 'Ball retroreflector optics', NRAO GBT Meno Series 148, 1-44.

URL: https://safe.nrao.edu/wiki/pub/GB/Knowledge/GBTMemos/GBT Memo_148.pdf

GOM (2010), 'Pontos - dynamic 3d analysis'.

Gorte, B. (2006), Advanced remote sensing: Image processing. Lecture notes Advance Remote Sensing of the master program Geomatcis at the faculty of Aerospace engineering Delft University of Technology.

Green, B. (2002), 'Canny edge detection tutorial'.

URL: http://www.pages.drexel.edu/weg22/can_tut.html

Haralick, R. M. and Shapiro, L. G. (1992), Computer and Robot Vision, Addison-Wesley Longman Publishing Co., Inc., Boston, MA, USA.

Hartley, R. I. and Zisserman, A. (2004), Multiple View Geometry in Computer Vision, second edn, Cambridge University Press, ISBN: 0521540518.

Hedrick, T. L., Usherwood, J. R. and Biewener, A. A. (2004), 'Wing inertia and whole-body acceleration: an analysis of instantaneous aerodynamic force production in cockatiels (nymphicus hollandicus) flying across a range of speeds', J Exp Biol 207, 1689-702.

URL: $\quad$ http://www.biomedsearch.com/nih/Wing-inertia-whole-bodyacceleration/15073202.html

Jolliffe, I. (2002), Principal Component Analysis, Springer Series in Statistics, 2nd edn, Springer.

Jonathan, B. (2010), 'How does an accelerometer work'. visited on: 11-102010.

URL: http://ezinearticles.com/?How-does-an-Accelerometer-Work? 
Bibliography

Karara, H. and Abdel-Aziz, Y. (1971), 'Direct linear transformation from comparator coordinates into object space coordinates in close-range photogrammetry', Proceedings of the Symposium on Close-Range Photogrammetry, American Society of Photogrammetry pp. 1-18.

Khoshelham, K. (2009), 'Lecture notes photogrammetry'. Master program Geomatcis at the faculty of Aerospace engineering Delft University of Technology.

Kilpatrick, J. M. and Markov, V. (2008), 'Matrix laser vibrometer for transient modal imaging and rapid nondestructive', SPIE 7098. 8th International Conference on Vibrtaion Measurements by Laser Techniques.

Kim, H. Y. and Arajo, S. A. (2007), 'Grayscale template-matching invariant to rotation, scale, translation, brightness and contrast', IEEE Pacific-Rim Symposium on Image and Video Technology 4872, 100-11.

Kolahi, A., Hoviattalabc, M., Rezaeian, T., Alizadeh, M. and Mokhtarzadeh, M. B. H. (2007), 'Design of a marker-based human motion tracking system', Elsevier 2, 59-76.

URL: $\quad h t t p: / / w w w . s c i e n c e d i r e c t . c o m / s c i e n c e / a r t i c l e / B 7 X M N$ 4NBXVXB-1/2/8bc0dc0b98e883f6d3b80d80ed3cf217

Luhmann, T., Robson, S., Kyle, S. and Harley, I. (2006), Close Range Photogrammetry Principles, Methods and Applications, Whittles Publishing.

Maas, H. G. (1992), 'High-speed solid state camera systems for digital photogrammetry', International Archives ofPhotogrammetry and Remote Sensing 119, 709-713.

Meng, F. (2009), Experiment proposal. Experiment setup information.

Moriyama, T., Kochi, N., Yamada, M. and Fukaya, N. (2008), 'Automatic target-identification with the color-coded-targets', The International Archives of the Photogrammetry, Remote Sensing and Spatial Information Sciences XXXVII, 39-44.

Ozbek, M., Rixen, D. J. and Verbruggen, T. W. (2010), 'Remote monitoring of wind turbine dynamics by laser interferometry: Phase1'.

PhotoCore (2010), 'Getting started with photocore live'.

URL: http://www.photocore.ch/live_gettingstarted.html

PhotoModeler (2010), 'Measuring and modeling the real world'.

URL: http://www.photomodeler.com/index.htm 
Rademakers, L., Verbruggen, T., van der Werff, P., Korterink, H., Richon, D., Rey, P. and F., F. L. (2004), 'Fiber optic blade monitoring', European Wind Energy Conference.

Rasmussen, F. (2003), 'Present status of aeroelasticity of wind turbines', Wind Energy 6, 213-228.

URL: http://adsabs.harvard.edu/abs/2003WiEn...6..213R

Riziotis, V. A., Voutsinas, S. G., Politis, E. S. and Chaviaropoulos, P. K. (2004), 'Aeroelastic stability of wind turbines: the problem, the methods and the issues', Wind Energy 7, 373 - 392.

Sensorland (2010), 'The strain gauge'. visited on: 11-10-2010.

URL: http://www.sensorland.com/HowPage002.html

Shannon, C. (1949), 'Communication in the presence of noise', Proceedings of the IRE 86, 447-457.

URL: http://www.stanford.edu/class/ee104/shannonpaper.pdf

Shortis, M. R., Clarke, T. A. and Short, T. (1994), Comparison of some techniques for the subpixel location of discrete target images, in S. F. ElHakim, ed., 'Society of Photo-Optical Instrumentation Engineers (SPIE) Conference Series', Vol. 2350 of Presented at the Society of Photo-Optical Instrumentation Engineers (SPIE) Conference, pp. 239-250.

URL: $\quad$ http://adsabs.harvard.edu/cgi-bin/nphbib_query?bibcode=1994SPIE.2350..239S

Shortis, M., Seager, J., Robson, S. and Harvey, E. (2003), Automatic recognition of coded targets based on a hough transform and segment matching, in 'Proceedings of Videometrics VII', Vol. 5013, pp. 202-208.

Teunissen, P. J. G. (2003), Adjustment Theory an Introduction, Mathematical Geodesy and Positioning, first edn, VSSD.

Tianshu, L., L. N. Cattafesta, I., R. H., R. and A. W., B. (2000), 'Photogrammetry applied to wind tunnel testing'.

Zheng, L., Wang, X., Khan, A., Vallance, R. R. and Mittal, R. (2009), 'A combined experimentalnumerical study of the role of wing flexibility in insect flight', 47th AIAA Aerospace Sciences Meeting Including The New Horizons Forum and Aerospace Exposition 382, 2.

URL: http://www.me.jhu.edu/fsag/Publications/Papers/AIAA-2009-382170\%5B1\%5D.pdf 


\section{Colophon:}

This document was typeset using $\mathrm{HT}_{\mathrm{E}} \mathrm{X} \pi-1.40 .10$ on Windows 7 using the Computer Modern family of typefaces, with TeXworks and TeXnicCenter as a front-end and BIBTEXfor bibliography management.

Most figures have been generated with MATLAB and edited using Inkscape. 


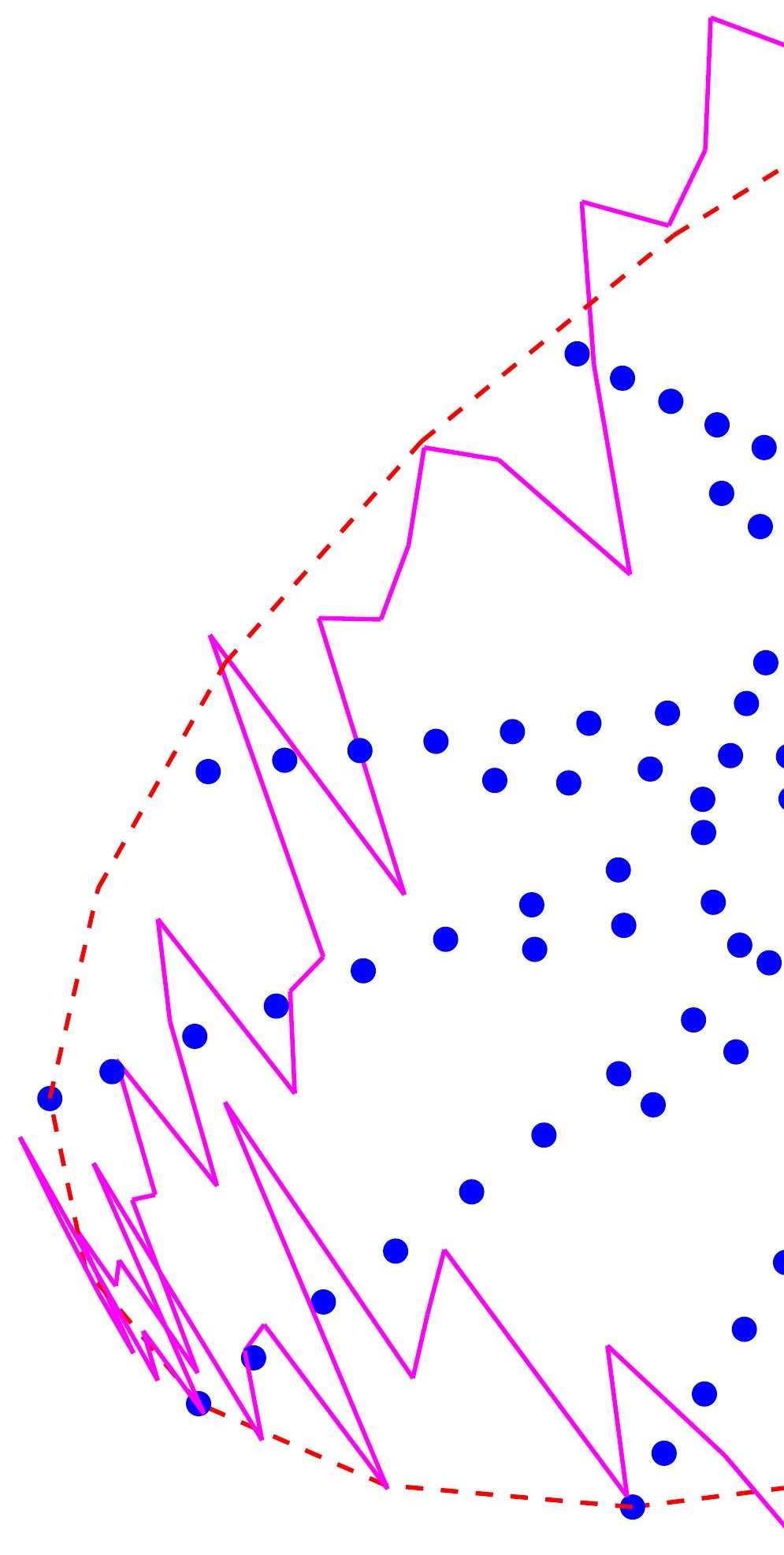

